\title{
On the History of Dibaryons and their Final Observation*
}

\author{
H. Clement \\ Physikalisches Institut der Universität Tübingen \\ and \\ Kepler Center for Astro and Particle Physics, University of Tübingen, \\ Auf der Morgenstelle 14, D-72076 Tübingen, Germany
}

December 15, 2016

\begin{abstract}
A review is given about the long-standing search for dibaryons, i.e. six-quark objects, from the early days until present, when the first dibaryon resonance has been established, which has the potential of constituting a compact six-quark object.
\end{abstract}

\section{Contents}

1 Introduction 3

2 Basics of the Baryon-Baryon System 4

2.1 The Nucleon-Nucleon System ........................... 4

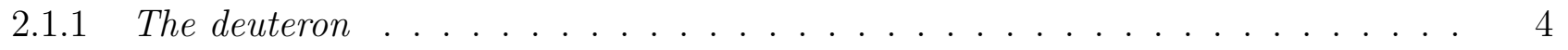

2.1.2 Dineutron and diproton. . . . . . . . . . . . . . . . . . 4

2.1.3 The virtual ${ }^{1} S_{0}$ state in the isovector nucleon-nucleon system . . . . . . . . . . 5

2.2 The Hyperon-Nucleon System . . . . . . . . . . . . . . . . . . 5

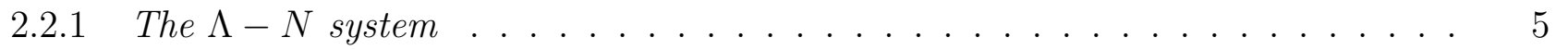

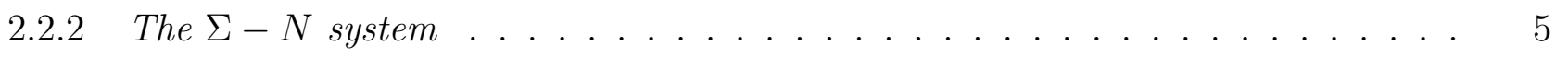

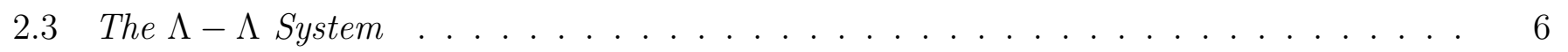

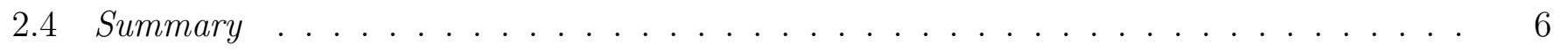

3 The Early Days of Dibaryon Searches 6

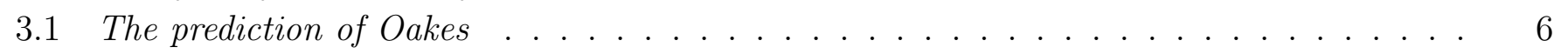

3.2 The Prediction of Dyson and Xuong . . . . . . . . . . . . . . . . . . 7

\footnotetext{
*To be published in Progress in Particle and Nuclear Physics 2017
} 
4 The Dibaryon Rush Era 7

4.1 Theoretical Predictions . . . . . . . . . . . . . . . . . . . 8

4.2 Experimental Searches for Narrow Dibaryons . . . . . . . . . . . . . . . . . . . . . 10

4.2 .1 Search in $N N$ and $\pi d$ scattering . . . . . . . . . . . . . . . . . . . . . . 10

4.2 .2 Search in pion production . . . . . . . . . . . . . . . . . 11

4.2 .3 Search in photo-induced reactions . . . . . . . . . . . . . . . . . 12

4.2.4 Search for super-narrow dibaryon resonances below the $N N \pi$ threshold . . . . . 14

4.2.5 Search in hyperon production and hyperon formation . . . . . . . . . . 15

4.3 Conclusions about this Period . . . . . . . . . . . . . . . . . . . . 17

4.4 A Possible Remnant from this Period - a Broad Resonance structure around the $\Delta N$ Threshold ........................... [17

5 An Intermezzo in Nuclei: The Pionic Double Charge Exchange Reaction 20 5.1 Yet Another Dibaryon Candidate: $d^{\prime}(2065) \ldots \ldots \ldots \ldots$. . . . . . . . . . . 22

6 More Recent Searches for Strange $(S=-1)$ Dibaryons

7 Status on the $H$ Dibaryon $(S=-2)$

8 The New Era of Exclusive and Kinematically Complete High-Statistics Measurements at CELSIUS and COSY 28

8.1 No signal for Narrow Resonances in the pp system . . . . . . . . . . . . . . . . 29

8.2 No Narrow Resonances in Hyperon Production - except of $\Sigma N$ cusp . . . . . . . . . . 29

8.3 No signal from the anticipated $N N \pi$ Resonance $d^{\prime}(2065) \ldots \ldots \ldots \ldots$. . . . . . . . . .

8.4 No dibaryon signal in pp-induced two-pion production . . . . . . . . . . . . . . . 34

9 Towards the First Extraordinary Dibaryon 37

9.1 Following a trace: the $A B C$ effect . . . . . . . . . . . . . . . . 37

9.2 Exclusive and Kinematically Complete Measurement of the Basic Double-Pionic Fusion Process .............................. 39

9.3 Search for the $d^{*}$ Resonance Structure in Isoscalar Non-Fusion Two-Pion Production . 43

$10 d^{*}(2380)$ - a Genuine Dibaryon Resonance 43

10.1 Former Occasions for Its Discovery . . . . . . . . . . . . . . . . . . . 47

$10.2 d^{*}(2380)$ in Nuclear Matter . . . . . . . . . . . . . . . . . . . . . . 47

$10.3 d^{*}(2380)$ in Theory . . . . . . . . . . . . . . . . . . . . . . . 48

10.4 The Branchings of the $d^{*}(2380)$ Decays . . . . . . . . . . . . . . . . 50

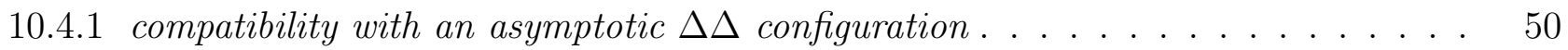

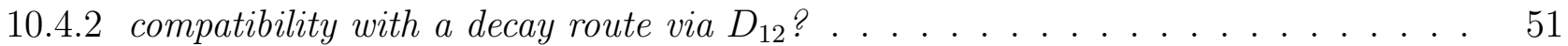

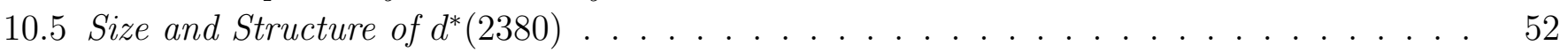

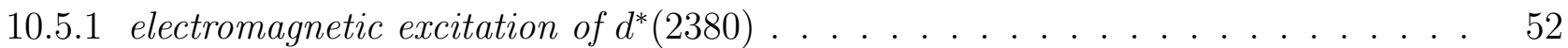

10.5.2 the ABC effect and the structure of $d^{*}(2380) \ldots \ldots \ldots \ldots \ldots$

11 Are There More Extraordinary Dibaryons? 54

11.1 Search for the Isospin $I=3$ State $D_{30} \ldots \ldots \ldots \ldots \ldots \ldots \ldots \ldots \ldots$

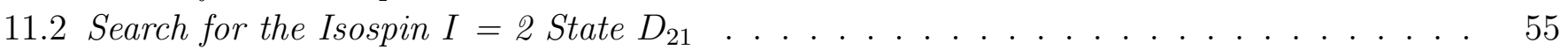

11.3 Renaissance of the $\Delta N$ Region: more Resonance Structures . . . . . . . . . . 56

11.4 Upcoming Results for the $\Omega^{-} p$ system $(S=-3) \ldots \ldots \ldots \ldots \ldots \ldots \ldots$

11.5 Are there Dibaryons in the Heavy-Quark Sector? . . . . . . . . . . . . . . . . . . . 58 


\section{Introduction}

Strictly speaking a dibaryon denotes just any object with baryon number $\mathrm{B}=2$. In this sense the first known dibaryon has been the deuteron discovered in 1932 by Urey, Brickwedde and Murphy [1]. In terms of quarks a dibaryon is composed of six valence quarks - or more generally speaking the number of quarks minus the number of antiquarks has to be six. It may be of molecular type, i.e. spatially extended with two well separated interacting quark bags as is the case for the well-known deuteron. Or - more exotic and hence more interesting - a dibaryon could be a spatially compact hexaquark object, where all six quarks sit in a single quark bag.

The long history of dibaryon searches dating back to the fifties has been a very changeful one with many ups and downs. Early predictions of a vast number of dibaryon states initiated endless experimental claims, but finally none survived careful experimental investigations. For a review about this dibaryon rush era, which lasted until the eighties, see, e.g. Refs. [2, 3, 4, 5, 6, 7, 8]. For more recent reviews from the theoretical point of view see, e.g., Ref. [9, 10].

Despite their long painful history dibaryon searches have recently received renewed interest, in particular by the recognition that there are more complex quark configurations than just the familiar $q \bar{q}$ and $q q q$ systems - in favor also of hidden-color aspects [11]. Recently two groups announced that lattice QCD calculations [12, 13, 14, 15] provide evidence for a bound $H$-dibaryon - as predicted by Jaffe [16] already in 1977. Nevertheless any experimental evidence for it is still pending despite the manyfold experimental efforts [17, 18, 19, 20, 21, 22, 23, 24, 25].

Recently the WASA-at-COSY collaboration has found that the double-pionic fusion reaction $p n \rightarrow$ $d \pi^{0} \pi^{0}$ proceeds dominantly via a resonance structure observed in the total cross section at $\sqrt{s}=2.37$ $\mathrm{GeV}$ with $\Gamma \approx 70 \mathrm{MeV}$ and $I\left(J^{P}\right)=0\left(3^{+}\right)$[26] - after first signs of it had been observed already at Uppsala by the CELSIUS/WASA collaboration [27]. Meanwhile nearly all possible decay channels have been investigated [28, 29, 30, in particular also the one into the elastic np channel. And new data on polarized $n p$ scattering in the region of interest exhibit a resonance pole in the coupled ${ }^{3} D_{3}-{ }^{3} G_{3}$ partial waves in accordance with the resonance hypothesis [31, 32]. This gives the first solid evidence for the existence of a non-trivial, possibly extraordinary dibaryon.

Since the measurements suggest this resonance to decay dominantly via an intermediate $\Delta \Delta$ system, it constitutes asymptotically a $\Delta \Delta$ system bound by nearly $100 \mathrm{MeV}$ - as predicted by Dyson and Xuong [33] already in 1964 and later-on also by Goldman et al. [34, who called it the "inevitable dibaryon"

$d^{*}$ due to its unique symmetry features. Most recent relativistic three-body calculations based on hadron dynamics [35, 36] as well as quark model calculations [37, 38, 39] succeeded to predict properly a number of characteristics of this resonance. The latter also postulate a substantial hidden-color component accounting in particular for the unusually narrow width of this resonance.

In the following we present a historical review about dibaryon predictions as well as experimental dibaryon searches. We start with some basics of the baryon-baryon system, before we enter the early days of dibaryon initiatives. Then we consider the dibaryon rush era initiated by Jaffe's prediction of the $H$ dibaryon, a bound $\Lambda \Lambda$ system. The deplorable experience with unjustified claims of dibaryon discoveries due to statistically poor, inclusive and/or kinematically incomplete measurements led in the nineties on the one hand to a big frustration about this subject, but on the other hand also to a restart of dedicated exclusive and kinematically complete high-statistics measurements, which will be the topic of the second part of this review and which finally lead to the first solid evidence for a non-trivial dibaryon resonance. Finally we give an outlook on future activities in this field. 
In the following we will adopt the convention of the Particle Data Group [40] and quote masses for simplicity in $\mathrm{MeV}$ instead of $\mathrm{MeV} / \mathrm{c}^{2}$. Also, angular momenta between baryons are denoted by capital letters, otherwise by small letters.

\section{Basics of the Baryon-Baryon System}

Naturally the quest for dibaryons concentrated first on the nucleon-nucleon $(N N)$ system, before lateron also the strangeness sector was explored.

\subsection{The Nucleon-Nucleon System}

\subsubsection{The deuteron}

As mentioned already in the introduction, formally the oldest known dibaryon is the deuteron. And until recently it has been the only safely discovered dibaryon at all.

Since its discovery in 1932 by Urey, Brickwedde and Murphy [1] in the atomic spectrum of hydrogen gas in a discharge tube, the spectroscopic quantities of the deuteron like mass, charge, spin-parity, dipole and quadrupole moments as well as form factors and structure functions have been measured with high precision.

The deuteron is a very loosely bound object with a binding energy of only $2.2 \mathrm{MeV}$ or $1.1 \mathrm{MeV} / \mathrm{A}$ - which has to be compared to the average binding energy of about $8 \mathrm{MeV} / \mathrm{A}$ in nuclei. Accordingly it possesses a very large charge radius of $2.1 \mathrm{fm}$ [41, 42], i.e. the centers of proton and neutron are on

the average further apart from each other then the range of the pion exchange $r \approx \frac{\bar{h} c}{m} \pi \approx 1.4 \mathrm{fm}$, which constitutes the longest range hadronic interaction in the $N N$ system.

Due to this large internucleon distance the pion exchange is the by far dominating interaction, which completely describes the asymptotic behavior of the deuteron. Hence the pioneering simple model descriptions of Hulthen [43] and Reid 44] have been already very successful in properly accounting for the asymptotic behavior of the deuteron wavefunction. Only at short distances in the neutron-proton overlap region the situation is not yet fully understood. In particular it is still an unsolved problem, how much of a hexaquark configuration within a compact six-quark bag is present in the deuteron. Whereas early theoretical work [45] on that claimed up to $1.5 \%$ six-quark content in the deuteron, more recent evaluations see this number near $0.15-0.30 \%$ [46, 47]. However, a recent dressed bag model calculation arrives again at $2-3 \%$ [48].

For the $\Delta \Delta$ component in the deuteron there exists an experimental upper limit of $0.4 \%$ at the $90 \%$ confidence level from neutrino-deuteron interaction studies [49, 50]. Theoretical estimates give contributions of the same order [50, 51].

\subsubsection{Dineutron and diproton}

The question, whether a stable diproton, i.e. a stable ${ }^{2} \mathrm{He}$ nucleus exists, may be solved easily by mass spectrometer measurements. With regard to the dineutron the experimental situation is not quite as easy, since it has no charge and hence also no atomic shell, which could reveal its existence or non-existence.

However, we know the answer to both questions from NN scattering experiments and successive partial-wave analyses. Of particular interest in this respect are the $L=0$ partial waves ${ }^{1} S_{0}$ and ${ }^{3} S_{1}$ with isospin 1 and 0 , respectively, most importantly their scattering lengths. If the scattering length is negative, then we have an attractive interaction, which however is not strong enough to create a $N N$ boundstate. Only, if the scattering length is positive and larger than the effective range of the interaction, then the $N N$ system possesses an boundstate in this particular partial wave. 
For the scattering length in the isoscalar ${ }^{3} S_{1}$ partial wave, which occurs only in the $n p$ system, a value of $\approx+5 \mathrm{fm}$ [52] has been obtained experimentally, i.e. this system has a boundstate, the well-known deuteron boundstate.

For the scattering length of the isovector ${ }^{1} S_{0}$ partial wave the experimental results are $\approx-18 \mathrm{fm}$ in case of $n n$ [53, 54] and $p p$ [55] scattering and $-24 \mathrm{fm}$ in case of $n p$ scattering [52, 56] - the difference being due to charge symmetry breaking. These results tell us that there is no boundstate in $n n$ and $p p$ systems.

\subsubsection{The virtual ${ }^{1} S_{0}$ state in the isovector nucleon-nucleon system}

Though the $N N$ interaction in the isovector ${ }^{1} S_{0}$ channel is too weak to produce a boundstate, it is strong enough to create at least a virtual state being unbound by merely $66 \mathrm{keV}$ [57]. In nuclear reactions experiments this state is sensed as a final state interaction (FSI) between emitted nucleons [58, 59]. There it shows up as a low-mass enhancement in $N N$ invariant-mass distributions - as depicted e.g. in Fig. 1.

\subsection{The Hyperon-Nucleon System}

For the Hyperon-Nucleon system there is naturally much less information available from experimental side, since hyperons are not stable. For the most long-lived hyperon, the only weakly decaying $\Lambda(1116)$, some kind of secondary beam may be obtained from $\Lambda$ production processes in nuclear targets, but this is still of very limited statistics and precision.

\subsubsection{The $\Lambda-N$ system}

Most information about the interaction between $N$ and $\Lambda$ may be obtained by considering the final-state interaction between both particles in production processes like $p p \rightarrow \Lambda p K^{+}$. There it shows up as an enhancement at the low-mass threshold in the $\Lambda N$ invariant-mass distributions - similar to the case of the $N N$ FSI. For an example see Fig. 7, where the $\Lambda N$ FSI is clearly seen as a strong enhancement at the $\Lambda p$ threshold. From analyses of the FSI enhancement and of the limited data on elastic $p \Lambda$ scattering a spin-averaged scattering length of $a_{\Lambda N} \approx-2 \mathrm{fm}$ has been deduced.

From precision measurements of this reaction with polarized beam it has been possible recently to resolve the spin-dependence of the scattering length and to extract model-independently the spin-triplet scattering length as $a_{t}=-2.3_{-1.39}^{+0.72}$ stat $\pm 0.6_{\text {syst }} \pm 0.3_{\text {theor }}$ fm [60], which agrees with $a_{t}=-1.6_{-0.8}^{+1.1} \mathrm{fm}$ from $p \Lambda$ elastic scattering [61]. For the spin-singlet scattering length a value of $a_{s}=-2.43_{-0.25}^{+0.16} \mathrm{fm}$ has been deduced [62]. These results are in agreement with model predictions based on meson exchange [63, 64] and chiral effective field theory [65].

The experimental results mean that the $\Lambda N$ interaction is attractive, but substantially less attractive than the $N N$ interaction, so that no boundstate can be formed, i.e., in particular there is no "strange" deuteron.

\subsubsection{The $\Sigma-N$ system}

The $\Sigma N$ interaction can be studied by hyperon-production reactions of the type $p p \rightarrow \Sigma^{0} p K^{+}$and $p p \rightarrow \Sigma^{+} p K^{0}$. Both reactions have been studied in exclusive and kinematically complete measurements at COSY-TOF [66, 67]. No sign of a significant $\Sigma p$ FSI has been observed. This means that the $\Sigma N$ interaction must be substantially weaker than the $\Lambda N$ interaction. 


\subsection{The $\Lambda-\Lambda$ System}

In heavy-ion collisions $\Lambda$ particles are produced abundantly, so that also the $\Lambda \Lambda$ FSI can be looked at. In measurements of $\mathrm{Au}-\mathrm{Au}$ collisions with the STAR detector at RHIC the $\Lambda \Lambda$ correlation function has been obtained [23]. Whereas in a first analysis a slightly repulsive interaction has been deduced, an improved analysis obtains a scattering length of $-1.2 \mathrm{fm}<a_{\Lambda \Lambda}<-0.5 \mathrm{fm}$ [24], which still would exclude a boundstate in the $\Lambda \Lambda$ system. However, if in this analysis so-called feed-down corrections are included, the limits relax to $a_{\Lambda \Lambda}>-1.2 \mathrm{fm}$, which does not completely rule out a boundstate - see the discussion about the $H$ dibaryon in sect. 7 .

\subsection{Summary}

Summarizing, in the baryon-baryon $(B B)$ system we have so far clear-cut experimental evidence only for a single boundstate, which is the deuteron groundstate known since 1932. In particular, there is no boundstate in the hyperon-nucleon system with strangeness $S=-1$. In the strangeness $S=-2$ sector the existence of a possible boundstate, the $H$ dibaryon, has not yet been ruled out completely at present.

\section{The Early Days of Dibaryon Searches}

The question, whether there are more eigenstates in the system of two baryons than just the ${ }^{3} S_{1}$ deuteron groundstate and the virtual ${ }^{1} S_{0}$ state, has been around in principle since the discovery of the deuteron in 1932. It became clear pretty soon that the loosely bound deuteron would not have any excited bound states. Hence the search for resonances focused soon on nucleon-nucleon collision energies close to the pion-production threshold and above, where new degrees of freedom come into play. Reports on such measurements [68, 69, 70] date back to the fifties, when suitable particle accelerators became available. In particular measurements of the reactions $p p \rightleftharpoons d \pi^{+}$indicated a resonant behavior with $I\left(J^{P}\right)=1\left(2^{+}\right)$right at the $\Delta(1232) N$ threshold with a width identical to that of the $\Delta(1232)$ resonance. We will come back to a detailed discussion of this dibaryon candidate in sect. 4.4.

\subsection{The prediction of Oakes}

Already before the publication of the quark model by Gell-Mann [71] Oakes considered the two-baryon system in a multiplet representation of $S U(3)$ following the so-called eightfold way [72]. This led to the prediction of 10 baryon-baryon states with the following hypercharge (Y) and isospin (I) combinations:

- 1 state with $(\mathrm{Y}, \mathrm{I})=(2,0)$ containing the baryon-baryon configuration $(n p)$,

- 2 states with $(\mathrm{Y}, \mathrm{I})=(1,1 / 2)$ containing the baryon-baryon configurations $(\Lambda N, \Sigma N)$,

- 3 states with $(\mathrm{Y}, \mathrm{I})=(0,1)$ containing the baryon-baryon configurations $(\Sigma \Lambda, \Sigma \Sigma, \Xi N)$ and

- 4 states with $(\mathrm{Y}, \mathrm{I})=(-1,3 / 2)$ containing the baryon-baryon configurations $(\Xi \Sigma)$.

In the limit of exact unitary symmetry, in which the masses of the eight baryons $N, \Lambda, \Sigma$ and $\Xi$ are degenerate, also the baryon-baryon forces are the same and hence the masses of these baryon-baryon states are degenerate. However, since this symmetry is broken, their masses are different, but may be calculated by use of a mass formula derived from this symmetry breaking.

Oakes identified the single state with a $n p$ configuration with the well-known deuteron. Since this has spin-parity $J^{P}=1^{+}$, also the other nine states should have the same quantum numbers.

By use of the Gell-Mann-Okubo [73, 74] mass formula and by identifying the $(Y, I)=(1,1 / 2)$ doublet with the $\Sigma N$ cusp at $2.13 \mathrm{GeV}$, which was already known at that time - see detailed discussions in 
sections 4.2 .5 and 8.2 - he predicted the $(Y, I)=(0,1)$ triplet and $(-1,3 / 2)$ quadruplet states to be at 2357 and $2564 \mathrm{MeV}$, respectively. Since these masses are substantially larger than the masses of their constituents, he concluded that these states are far from being bound states, i.e., could only be resonance states.

\subsection{The Prediction of Dyson and Xuong}

The quest for dibaryon states was reinforced, when it got apparent that baryons and mesons contain substructures, the quarks, and QCD does not prohibit colorless multiples of three quarks, in particular does not forbid quarks in a colorless six-pack. In fact, shortly after Gell-Mann's famous publication [71] of the quark model in 1964 Dyson and Xuong [33] demonstrated that SU(6) symmetry provides a multiplet of six non-strange dibaryon states denoted by $D_{I J}$ with $I J=01,10,12,21,03$ and 30 as given in Table 1.

The first three states of their sextet Dyson and Xuong identified as the deuteron groundstate, the virtual ${ }^{1} S_{0}$ isovector state - known at that time already from the final-state interaction in low-energy nucleon-nucleon scattering - and an $I\left(J^{p}\right)=1\left(2^{+}\right)$state right at the $\Delta N$ threshold, for which first experimental indications had been available already at that time - see discussion in Ref. [33. This way they fixed all parameters of their mass formula allowing the prediction of masses for the remaining three higher-lying states. By identifying the first two states with the deuteron groundstate and the ${ }^{1} S_{0}$ virtual state with both having roughly the same mass the predicted dibaryon masses become isospin independent, i.e. $m\left(D_{21}\right)=m\left(D_{12}\right)$ and $m\left(D_{30}\right)=m\left(D_{03}\right)$.

Therefore $D_{21}$ is expected to be situated similar to $D_{12}$ near the $\Delta N$ threshold with a mass around $2160 \mathrm{MeV}$. Due to its isospin $I=2$ this state is decoupled from the $N N$ system and hence can be produced only associatedly in $N N$ collisions, e.g., in two-pion production via the process $p p \rightarrow$ $D_{21}^{+++} \pi^{-} \rightarrow p p \pi^{+} \pi^{-}$. For a more detailed discussion about this proposed state see sect. 11.2.

According to the mass formula the next higher-lying dibaryon state, $D_{03}$, is expected to be at $2350 \mathrm{MeV}$, i.e. about $110 \mathrm{MeV}$ below the $\Delta \Delta$ threshold. Due to its quantum numbers it should asymptotically - i.e. at large distances as an intermediate step in its decay or formation - conform to an isoscalar $\Delta \Delta$ configuration with $J^{P}=3^{+}$. Most favorably this means two spin aligned $\Delta$ particles in relative $S$-wave. Since this state is isoscalar, it should couple to the $n p$ system and be sensed in its ${ }^{3} D_{3}$ partial wave. And because the $\Delta$ excitation is highly inelastic due to its preferred decay into $N \pi$, the $D_{03}$ excitation will be highly inelastic, too. Hence it appears to be more favorable to sense this state in the process $n p \rightarrow D_{03} \rightarrow N N \pi \pi$ rather than searching in $n p$ scattering. In fact, such a state was observed experimentally just very recently. And indeed, it was first found in the two-pion production reaction $n p \rightarrow d \pi^{0} \pi^{0}$, before it was confirmed in $n p$ scattering - see sections 9 and 10 .

Finally, the last state, $D_{30}$, predicted by Dyson and Xuong with quantum numbers just mirrored to $D_{03}$ constitutes a really exotic one. Its highest charge state $D_{30}^{++++}$consists of six up quarks, which asymptotically yield a $\Delta^{++} \Delta^{++}$configuration. Due to its large isospin this state needs to be produced associatedly in $N N$ collisions with at least two other pions, e.g in a four-pion production process of the form $p p \rightarrow D_{30}^{+++} \pi^{-} \pi^{-} \rightarrow \Delta^{++} \Delta^{++} \pi^{-} \pi^{-} \rightarrow p p \pi^{+} \pi^{+} \pi^{-} \pi^{-}$. For a search for this state see sect. 11.1.

\section{The Dibaryon Rush Era}

As we have seen, the first three states in Dyson's dibaryon sextet constitute quite conventional states, where the two bags containing three quarks each do not overlap markedly. Maybe it was this fact that this work did not find overwhelming attention - though as we will see below it turns out now to have quite some predictive power in view of the first observation of a non-trivial dibaryon resonance.

A real dibaryon rush started, when Jaffe 1977 [16] predicted the so-called $H$ dibaryon, a hadronically 
Table 1: Prediction of Dyson and Xuong [33] about a sextet of non-strange dibaryon states based on $S U(6)$ symmetry. The states are denoted by $D_{I J}$, where $I$ denotes the isospin and $J$ the total spin of the state. Given are the associated asymptotic baryon-baryon $(B B)$ configurations and the masses calculated from symmetry breaking and by identifying the two lowest-lying states with the deuteron groundstate and the virtual ${ }^{1} S_{0}$ state.

\begin{tabular}{ccllll}
\hline notation & I & $\begin{array}{c}\text { J } \\
\text { asymptotic baryon-baryon } \\
\text { configuration }\end{array}$ & mass (formula) & $\begin{array}{l}\text { mass (value) } \\
(\mathrm{MeV})\end{array}$ \\
\hline$D_{01}$ & 0 & 1 & deuteron & $\mathrm{A}$ & 1876 \\
$D_{10}$ & 1 & 0 & ${ }^{1} S_{0} N N$ virtual state & $\mathrm{A}$ & 1876 \\
$D_{12}$ & 1 & 2 & $\Delta N$ & $\mathrm{~A}+6 \mathrm{~B}$ & 2160 \\
$D_{21}$ & 2 & 1 & $\Delta N$ & $\mathrm{~A}+6 \mathrm{~B}$ & 2160 \\
$D_{03}$ & 0 & 3 & $\Delta \Delta$ & $\mathrm{A}+10 \mathrm{~B}$ & 2350 \\
$D_{30}$ & 3 & 0 & $\Delta \Delta$ & $\mathrm{A}+10 \mathrm{~B}$ & 2350 \\
\hline
\end{tabular}

bound $\Lambda \Lambda$ system containing two strange quarks. This initiated a flood of further predictions on a multitude of states in all kind of baryon-baryon systems, which in turn initiated world-wide experimental searches for bound and unbound dibaryon states. As a consequence a huge number of claims have been made. However, finally not a single one survived a critical inspection.

A major reason for this striking failure was certainly the insufficient quality of experimental data obtained by use of inadequate instrumental equipment. Other reasons may concern the wrong choice of reaction and/or energy region, where searches have been conducted. For reviews on this epoch see Refs. [2, 3, 4, 7, 8]. For a critical, but also very amusing review of this epoch see, e.g. K. K. Seth [5, 6]. For a more recent review from the theoretical point of view see also A. Gal [9].

\subsection{Theoretical Predictions}

Triggered by Jaffe's prediction [16] of a bound $\Lambda \Lambda$ system many other theoretical predictions appeared in the following years based on QCD-inspired models like bag, potential, string or flux-tube models for the six-quark system [75, 76, 77, 778, 79, 80, 81, 82, 83, 84, 85, 86, 87, 88, 89, 90, 91, 92, 93, 94, 95, 96, 34, 97, 98], models of just the hadronic baryon-baryon interaction without any explicit quark-degrees of freedom [99, 100, 101, 102, 103, 104, 105, 106] or just symmetry considerations based on SU(3) [107]. Most recently also lattice QCD calculations [12, 13, 14, 15] joined this subject - see discussion in section 7.

In general, the QCD-based or -inspired model calculations make use of the color-magnetic interaction between quarks giving rise to hyperfine splitting:

$$
v_{\text {color }- \text { mag }}=-\sum_{i<j}\left(\lambda_{i} \cdot \lambda_{j}\right)\left(\sigma_{i} \cdot \sigma_{j}\right) v\left(r_{i j}\right)
$$

where $\lambda_{i}$ and $\sigma_{i}$ denote color and spin operators, respectively, of the quark i and $v\left(r_{i j}\right)$ is a flavor conserving short-range interaction between the quarks $\mathrm{i}$ and $\mathrm{j}[9]$.

As pointed out by Jaffe and others, the color-magnetic interaction is most attractive in the flavorsinglet state with $I\left(J^{P}\right)=0\left(0^{+}\right)$and $S=-2$, the $H$ dibaryon, the wavefunction of which corresponds asymptotically to baryon-baryon configurations of $\Lambda \Lambda, \Xi N$ and $\Sigma \Sigma[93$.

In this concept the leading dibaryon candidates having the underlying baryons in relative $S$ wave are states with [9, 93]:

- $S=0, I\left(J^{P}\right)=0\left(3^{+}\right)$and $B B$ structure $\Delta \Delta$, 
- $S=-1, I\left(J^{P}\right)=1 / 2\left(2^{+}\right)$and $B B$ structure $\Sigma^{*} N$ and $\Sigma \Delta$,

- $S=-2, I\left(J^{P}\right)=0\left(0^{+}\right)$with $B B$ structure $\Lambda \Lambda, \Xi N$ and $\Sigma \Sigma$ (the $H$ dibaryon) and

- $S=-3, I\left(J^{P}\right)=1 / 2\left(2^{+}\right)$with $B B$ structure $\Omega N, \Xi^{*} \Sigma, \Xi^{*} \Lambda$ and $\Xi \Sigma^{*}$,

where $B B$ means the baryon-baryon configuration, which is asymptotically closest to the dibaryon state.

The results based on eq. (1) assume unbroken $S U(3)$ symmetry. However, realistic considerations, which account for symmetry breaking, lead to significant changes in the predicted dibaryon masses.

With respect to a possible experimental observation of dibaryon resonances, predictions of states with low masses are particular interesting because of their expected narrow width.

The Nijmegen group [75, 76, 77, 78, well-known for their partial-wave analyses of $N N$ scattering and the therefrom derived Nijmegen potential, predicted a multitude of dibaryon resonances both in non-strange and strange sectors. They introduced an elongated bag allowing for finite orbital angular momenta between delocalized quark clusters in the partitions $q^{2}-q^{4}$ and $q-q^{5}$. As a consequence they obtained a multitude of dibaryon states both for non-strange and strange sectors. In particular, they predicted that the lowest-lying states with masses around $2.11 \mathrm{GeV}$ would be $N N$-decoupled due to their quantum numbers. Because of that they were expected to have particular narrow widths. For a discussion of those states see section 5 .

Most calculations agree insofar as they predict no dibaryons with masses below the $N N \pi$ threshold thus allowing them to decay still hadronically. The possible existence of an isotensor $N N \pi$ bound state is based on the hope that the attractive $\pi N$ interaction in the $P_{33}$ partial wave $(\Delta$ channel) might provide enough attraction to bind the barely unbound isovector $N N$ system - constituting thus a deeply bound $\Delta N$ system. Whereas early calculations for this scenario [75, 103, 104, 105, did not support the existence of such states, Garcilazo [101] did find theoretical evidence for a $n n \pi^{-}$boundstate in three-body Faddeev calculations, if the pion-nucleon interaction is sufficiently short-ranged, which, however, could not be decided given the uncertainty in our knowledge of the short-range part. By use of a phenomenological $\Delta$ model Dillig [106] predicted an $I\left(J^{P}\right)=2\left(1^{+}\right)$state at $2020 \mathrm{MeV}$, i.e. just barely unbound with a width of $\Gamma=1 \mathrm{MeV}$.

The Los Alamos theory group predicted dibaryons in the $\Omega N$ system, which could be so deeply bound that they would be stable with respect to strong decay [96. They also showed that predictions about the binding energy of the $H$ dibaryon critically depend on the detailed dynamics of the model under consideration. They rather emphasized the particular importance of the "inevitable" dibaryon, as they called it [34] and argued that certain basic features common to all models based on one-gluon exchange and confinement lead unavoidably to the prediction of a non-strange dibaryon resonance $d^{*}$ with $I\left(J^{P}\right)=0\left(3^{+}\right)$due to its special symmetry. Being asymptotically a bound $\Delta \Delta$ state it coincides with the $D_{03}$ state predicted by Dyson and Xuong. However, in the Los Alamos calculations it appears to be very deeply bound by nearly $400 \mathrm{MeV}$. In contrast, the MIT and cloudy bag model calculations [76, 79, 80] obtained for it binding energies relative to the $\Delta \Delta$ threshold of about $100 \mathrm{MeV}$, i.e. close to the value predicted before by Dyson and Xuong.

Mulders and Thomas [79] as well as Saito [80] demonstrated that pionic corrections ("pion cloud") do not have large influence on the mass of the "inevitable dibaryon", but large impact on the mass of the $H$ dibaryon pushing up its mass, possibly even into the unbound region. Oka, Shimizu and Yazaki showed that also in the non-relativistic quark cluster model the $H$ dibaryon gets unbound $[92$. Aerts and Dover [82] proposed the double-strangeness-exchange reaction as particularly suited for the $H$ search and calculated cross sections expected for the $K^{-3} \mathrm{He} \rightarrow H n K^{+}$reaction. 


\subsection{Experimental Searches for Narrow Dibaryons}

The prediction of a copious number of dibaryon states in strange and non-strange sectors initiated a rush of experimental searches for such states. Naturally, the search for narrow dibaryons was particularly attractive for two reasons.

First, a narrow resonance structure is much easier to discriminate experimentally against the background from conventional hadronic processes. The latter will dominate the considered reaction in general and will show also resonance-like structures due to hadronic excitations of baryons and mesons during the course of the reaction process. Since the widths of typical mesonic and baryonic resonances is about $120 \mathrm{MeV}$ and higher, observation of much narrower structures points to a very interesting exotic process.

Second, in order to have a small width, the decay of a dibaryon must be hindered either

- due to its exotic internal quark structure, which provides no large overlap with the asymptotic hadronic configuration of its decay products, and / or

- due to its quantum numbers, which hinder or even forbid the decay into lower-lying hadronic channels, and / or

- due to its mass being below some elsewise favored particle emission thresholds or at least nearby.

E.g., if the mass of the $H$ dibaryon is below the $\Lambda \Lambda$ mass, it can decay only by weak interaction and hence has a very narrow width. However, if its mass is above this threshold, it may decay by strong interaction in a fall-apart decay into $\Lambda \Lambda$ without any hindrance and hence have a very large width typical for hadronic $S$-wave decays.

Similarly, the non-strange $N N$-decoupled states $I\left(J^{P}\right)=0\left(0^{-}\right)$and $0\left(2^{-}\right)$predicted by Mulders et al. to lie above the $N N \pi$ threshold, should still have a fairly narrow width, since their decay into the $N N$ channel is forbidden due to their quantum numbers. In the $N N$ system such quantum number combinations are not allowed because of the Pauli principle.

\subsubsection{Search in $N N$ and $\pi d$ scattering}

From the experimental point of view the easiest channels for the dibaryon search to access with high precision are elastic proton-proton and pion-deuteron scattering. The latter got possible with the availability of high-quality and high-intensity $\pi^{+}$and $\pi^{-}$beams of kinetic energies up to $T_{\pi}=500 \mathrm{MeV}$ at the pion factories LAMPF, TRIUMF and PSI (formerly SIN), partly in combination with polarized deuteron targets.

These installations also allowed precise measurements of $p p$ scattering up to $T_{p}=800 \mathrm{MeV}$ with polarized beam and/or target. Still higher beam energies have been reached, e.g., in Gatchina, Dubna, Argonne National Lab, KEK and Saclay.

In order to access the $n p$ system experimentally one has to either produce explicitly a secondary neutron beam or utilize the quasifree reaction process $p+d \rightarrow(p+n)+p_{\text {spectator. }}$ In both cases deuterons are generally used as the provider of neutrons. The quasifree process has proven to work very well at beam energies of several hundred $\mathrm{MeV}$ and above. If the spectator proton is also measured in a kinematically complete experiment, then the Fermi motion within the deuteron can be utilized to measure the energy dependence of the observables over quite some range of energies simultaneously.

By the mid-nineties many thousands of experimental data points for differential and total cross sections as well as for polarization observables were obtained both for $p p, n p$ and $\pi d$ scattering. For $p p$ scattering they covered the beam energy region up to $1.6 \mathrm{GeV}$ (corresponding to a total center-of-mass energy of $\sqrt{s}=2.56 \mathrm{GeV})$, for $n p$ scattering up to $1.3 \mathrm{GeV}(\sqrt{s}=2.44 \mathrm{GeV})$ and for $\pi d$ scattering up to $500 \mathrm{MeV}(\sqrt{s}=2.44 \mathrm{GeV})$. 
These data were collected by centers conducting partial-wave analyses of these data, see, e.g. Refs. [108, 109, 110, 111 - the most prominent being the SAID data analysis center at Washington, DC. All partial-wave analyses give comparable results, in particular they give no evidence for resonances with the exception of the energy region around the $\Delta N$ threshold, which will be discussed in detail in section 4.4 .

For a review of odd structures in $N N$ scattering observables and their possible relation to dibaryons see, e.g. the review by Yokosawa [2] about the experimental polarized beam program at Argonne.

There also two anomalies have been observed in the difference $\Delta \sigma_{L}$ between $p p$ total cross sections for pure helicity states [112] at $\sqrt{s}=2.7-2.9 \mathrm{GeV}$, i.e. in the mass range, where there have been predictions for dibaryon states by LaFrance and Lomon [85, 86, 87]. At the lower energy also some narrow structure in the spin correlation observable $A_{00 n n}$ was reported from measurements at Saclay [113, which would correspond to a resonance mass of $2735 \mathrm{MeV}$ and an estimated width of $17 \mathrm{MeV}-$ in agreement with the predictions of Refs. [85, 86, 87] for a $I\left(J^{P}\right)=1\left(0^{+}\right)$state based on cloudy bag and R-matrix calculations.

\subsubsection{Search in pion production}

Single-pion production has been the first reaction process to look for dibaryons, since it was hoped that exotic processes would be sensed particularly well, if pionic degrees of freedom were explicitely involved. Indeed, as already mentioned, the $p p \rightarrow d \pi^{+}$reaction was used already in the fifties to search for signals from dibaryons [68, 70]. With the availability of pion beams at the pion factories the reverse reaction $\pi^{+} d \rightarrow p p$ got easily accessible and hence a wealth of data on this reaction up to $T_{\pi}=500 \mathrm{MeV}(\sqrt{s}=$ $2.44 \mathrm{GeV}$ ) stem from measurements at LAMPF, TRIUMF and PSI (formerly SIN). For higher energies measurements of $p p \rightarrow d \pi^{+}$reaction had to be conducted at appropriate proton accelerators.

The data for pion energies up to $T_{\pi}=500 \mathrm{MeV}$ have been accumulated in the SAID database amounting to several thousands of experimental data for total and differential cross sections as well as polarization observables. The partial-wave analysis [114] of these data does not give any hints for narrow resonances. However, in the $\Delta N$ threshold region the analysis exhibits pronounced loopings of particular partial waves in the Argand diagram - which usually is a clear signature of resonance phenomenon. For a detailed discussion on that see section 4.4 .

Experimentally more demanding is the study of pion production in reactions, where three or more particles are emitted. In such cases single-arm experiments, as usually used in two-body reactions, are no longer sufficient to record a reaction in an exclusive and kinematically complete way. Hence experimental setups with a single detector arm, e.g., a magnetic spectrometer, can measure such reactions only inclusively with the consequence of insufficient information for a proper analysis of the data, which in addition contain a much higher amount of background. To the contrary, bubble-chamber measurements may record all emitted particles, if they are charged - but they usually have the problem of low statistics.

Hence it is of no surprise that a number of dibaryon claims have been made resting upon the observation of some intriguing structures in the observables, which, however, could not be verified by other dedicated measurements.

E.g., Troyan and Pechenov found 17 narrow, only few MeV broad structures in a $p p$ invariant-mass spectrum, which - in order to increase statistics - they constructed out of many separate bubblechamber measurements of the reactions $n p \rightarrow p p \pi^{-}, n p \rightarrow p p \pi^{-} \pi^{0}, n p \rightarrow p p \pi^{0} \pi^{-}, n p \rightarrow p p \pi^{+} \pi^{-} \pi^{-}$ and $n p \rightarrow p p \pi^{+} \pi^{-} \pi^{-} \pi^{0}$ at neutron beam momenta between $1.26-5.24 \mathrm{GeV} / \mathrm{c}$ [115] - see Fig. 1. They associated these structures with 17 narrow dibaryons with masses in the range $1886-2282 \mathrm{MeV}$, though their statistical significance fully depended on the choice of conventional background assumed in the analysis. In fact, a subsequent exclusive high-resolution and high-statistics measurement of the $n p \rightarrow p p \pi$ reaction at ITEP [116] revealed that there were no narrow structures in the mass range up to $2170 \mathrm{MeV}$ except of the threshold structure due to the $p p$ final-state interaction caused by the 
well-known virtual ${ }^{1} S_{0}$ state, see section 2.3 .

The same bubble-chamber setup at Dubna has also been used to search for narrow $I=2$ dibaryon resonances in the reaction $n p \rightarrow p p \pi^{+} \pi^{-} \pi^{-}$. In the obtained $p p \pi^{+}$-invariant mass distribution eight narrow structures have been identified and claimed to belong to narrow $I=2$ dibaryon resonances [117.

Another claim for dibaryons in this mass range comes from inclusive, though high-statistics singlearm magnetic spectrometer measurements at Saclay [118]. Investigating the reactions of type $p p \rightarrow$ $p \pi^{+} X$ at beam energies of $T_{p}=1.5-2.1 \mathrm{GeV}$ three small structures were identified and associated with narrow dibaryons of masses between 2050 - $2150 \mathrm{MeV}$. In yet another experiment at Saclay a small oscillatory pattern has been observed in the tensor analyzing power and associated with a narrow dibaryon at $1945 \mathrm{MeV}$ [119.

Pion-production in nuclei also has been investigated with respect to possible dibaryon signals. Remarkably, several inclusive experiments conducted at Dubna [120], Saclay [121] and Moscow [122] noted a narrow, only a few $\mathrm{MeV}$ broad resonance structure at a mass of about $2220 \mathrm{MeV}$ in proton initiated subthreshold $\pi^{+}$production in nuclei, whereas this structure was not observed at TRIUMF in $\pi^{+}$and $\pi^{-}$production [123] as well as in $\pi^{0}$ production at Saclay [124]. The non-observation in the latter case could have been due to the insufficient energy resolution in that experiment. At first this structure was associated with the ${ }^{3} F_{3}$ dibaryon resonance candidate formed by in-medium $p p$ collisions, but it was soon realized that Fermi-motion would have necessarily smeared out such a narrow resonance as observed. For other hypotheses see, e.g. the discussion in Ref. [124]. Kurepin and Oganessian put the idea forward that a $I\left(J^{P}\right)=1\left(2^{+}\right) \Delta \Delta$ excitation shifted down in energy due to the nuclear medium might be the reason for this narrow structure [125].

A LAMPF experiment looking for very low-lying dibaryons in the $\pi^{+} d \rightarrow p p$ reaction found no evidence for such states [126].

\subsubsection{Search in photo-induced reactions}

Already as early as in the sixties it was noted that the proton polarization in deuteron photo-disintegration $\gamma d \rightarrow \overrightarrow{p n}$ shows a sharp increase - in particular at angles around $90^{\circ}$, if the incident photon energy is increased beyond $350 \mathrm{MeV}$ [127, 128]. A model assuming $\Delta$ excitation of one of nucleons in the struck deuteron could accommodate the data taken at lower energies, but not those taken at 400 and 500 $\mathrm{MeV}$.

In the seventies measurements of this particular reaction were extended up to $700 \mathrm{MeV}$ photon energy using Bremsstrahlung photons produced by electrons accelerated in the synchrotron at Tokyo. As a result it was observed that the polarization at $90^{\circ}$ starts rising at $300 \mathrm{MeV}$, reaches a maximum around $500 \mathrm{MeV}$ and starts dropping above $550 \mathrm{MeV}$ describing thus a resonance-like excitation function [129]. It was suggested that this behavior could be due to the excitation of a dibaryon resonance with a mass of $2380 \mathrm{MeV}$ and preferably with quantum numbers $I\left(J^{P}\right)=0\left(3^{+}\right)$as predicted in 1964 by Dyson and Xuong [33] and - in close connection with the Bremsstrahlung measurements - by Kamae and Fujita [99] based on a non-relativistic one-boson exchange potential model. Subsequent measurements improving the data base allowed for resonance fitting, which led to the assignment of several broad resonances with widths of $200 \mathrm{MeV}$ and beyond [130, 131, 132].

Another intriguing structure in inclusive photo-induced pion production on the deuteron was reported from Saclay measurements, where a bumpy structure was observed in the energy dependence of the cross section around $E_{\gamma}=400 \mathrm{MeV}$, which was associated with a dibaryon of mass $2230 \mathrm{MeV}[133$. Subsequent exclusive high-resolution measurements of the $\gamma d \rightarrow p p \pi^{-}$reaction carried out at Bonn demonstrated that the $p p \pi^{-}$invariant mass distribution is flat showing no evidence for any dibaryon in the mass range 2160 - $2320 \mathrm{MeV}$ [134] - in agreement with corresponding pion-induced measurements carried out at Saclay [135] and SIN [136]. However, the Bonn data surprisingly revealed a small narrow 

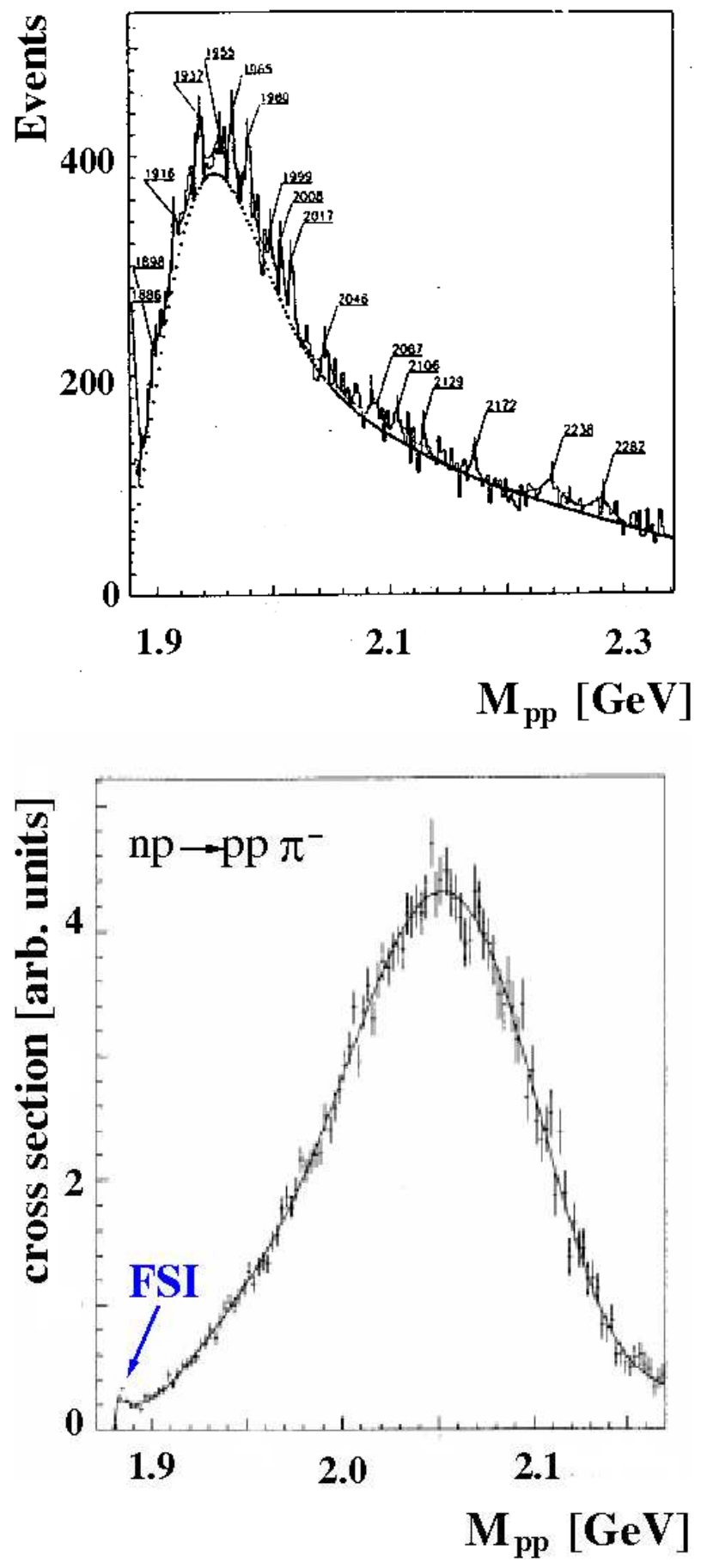

Figure 1: Distribution of the $p p$-invariant mass $M_{p p}$ as observed in $n p$-initiated single-pion production by the JINR bubble-chamber at Dubna [115] (top) and the magnetic spectrometer setup at ITEP [116] (bottom), respectively. Whereas in the Dubna spectrum, which includes also events from multi-pion production, 17 narrow spikes above an assumed smooth background (dotted line) have been assigned to narrow dibaryon resonances, the ITEP spectrum shows no statistically significant narrow structures except of the well-known enhancement at the $p p$ threshold due to the $N N$ FSI. From Refs. [115, 116]. 
structure in the $p p$ invariant-mass spectrum at $2014 \mathrm{MeV}$, which coincides right with the $p p \pi^{-}$threshold [137.

In CLAS measurements of the $\gamma d \rightarrow d \pi^{0}$ reaction at JLAB a resonance-like structure was observed at backward angles at $E_{\gamma}=700 \mathrm{MeV}$, which could be explained by $\eta$ excitation in the intermediate state connected to the $N^{*}(1535)$ baryon resonance [138].

Another search by using the $\gamma d \rightarrow \pi^{0} X$ reaction has been carried out at MAMI achieving an energy resolution of $0.8 \mathrm{MeV}$. Again no statistically significant narrow structure has been found, which could signal an isoscalar or isovector dibaryon resonance in the mass range below $2100 \mathrm{MeV}$ [139] - see also section 5 .

\subsubsection{Search for super-narrow dibaryon resonances below the $N N \pi$ threshold}

For dibaryons below the pion emission threshold the only hadronic decay channel is $N N$. However, if the quantum numbers of the dibaryon state are Pauli forbidden in the $N N$ system, i.e. if they are of the type $I\left(J^{P}\right)=0\left(0^{+}\right), 0\left(0^{-}\right), 0\left(2^{-}\right), 0\left(4^{-}\right), \ldots$ or $I\left(J^{P}\right)=1\left(1^{+}\right), 1\left(3^{+}\right), 1\left(5^{+}\right), \ldots$ or even of type $I\left(J^{P}, s\right)=1\left(1^{-}, 0\right)$, where $s$ denotes the internal spin, then such dibaryons can not decay into the $N N$ system - only electromagnetically by $\gamma$ emission. Therefore the decay widths of such dibaryons should be tiny. They have been estimated for some examples by Fil'kov [140, 141]. In an inclusive measurement of the $p d \rightarrow p X$ reaction at the Moscow Meson Facility three narrow peaks have been observed and associated with supernarrow dibaryons of masses 1904, 1926 and $1942 \mathrm{MeV}$ [142, 143]. Support for those lines has been found in an analysis of LEGS data on the $\vec{\gamma} d \rightarrow n n \pi^{+} \gamma$ reaction [144]. However, the mass range 1896 - $1914 \mathrm{MeV}$ was excluded later-on by inclusive measurements of the $p d \rightarrow p d X$ and $p d \rightarrow p p X$ reactions at Osaka [145, 146].

Similarly Gerasimov and Khrykin proposed Bremsstrahlung connected production of such dibaryons, in particular favoring the $p p \rightarrow p p \gamma \gamma$ reaction [147]. Indeed some bumpy structure corresponding to a dibaryon mass of about $1920 \mathrm{MeV}$ was recorded in a subsequent inclusive experiment at Dubna having a setup composed of just two photon detectors left and right of the beam-pipe [148, 149]. A followup measurement claimed a signal corresponding to a dibaryon mass shifted to $1956 \mathrm{MeV}$ [150, 151]. However, an exclusive and kinematically complete high-resolution experiment of the $p p \rightarrow p p \gamma \gamma$ reaction at CELSIUS, Uppsala utilizing the WASA detector found no evidence for any dibaryon resonance in the mass range $1900-1960 \mathrm{MeV}$ [420].

Still more exotic would be bound $p p \pi^{+}$and $n n \pi^{-}$systems. Such configurations would be members of an isotensor multiplet and could even not decay electromagnetically into the $N N$ system - only by weak interaction. Of these two charge states the $n n \pi^{-}$configuration would be bound even more likely, since there is no repulsive Coulomb interaction in this system. The possibility that these systems may be bound is based on the assumption that - similar to the case in nuclei, where ${ }^{3} \mathrm{H}$ and ${ }^{3} \mathrm{He}$ are bound more strongly than the deuteron - the attractive $\pi N$ interaction in the $\Delta$ channel will cause the barely unbound isovector $N N$ system to get bound. Experimentally this scenario can be studied very well at pion factories by using $\pi^{+}$and $\pi^{-}$beams and measuring the pionic double charge exchange reaction on the deuteron, i.e. $\pi^{+} d \rightarrow\left(p p \pi^{+}\right) \pi^{-}$and $\pi^{-} d \rightarrow\left(n n \pi^{-}\right) \pi^{+}$. By measuring the pion emitted associatedly with the $N N \pi$ system - preferably by means of a high-resolution magnetic spectrometer - its missing mass spectrum may display a peak outside the kinematic range for an unbound $N N \pi$ system, if a bound $N N \pi$ system exists. In a series of such experiments at LAMPF, PSI and TRIUMF no significant signs of $p p \pi^{+}$and $n n \pi^{-}$bound states have been found [153, 154, 155, 156, 157, 158]. For the analysis of such experiments concerning the possibility to observe narrow dibaryons see Ref. [159]. 
Early searches for dibaryons in the strange sector have been carried out in $K^{-} d$ interactions at rest, later also with low-energetic $K^{-}$beams hitting a deuteron target [160, 161, 162, 163, 164, 165, 166, 167]. The reaction looked at was $K^{-} d \rightarrow \Lambda p \pi^{-}$. With the exception of Ref. [167, which reports on an inclusive measurement by use of a magnetic spectrometer, bubble chambers were used for the detection of the reaction ejectiles. The use of bubble chambers allowed the detection of all charged ejectiles. In the case that also the $\Lambda \rightarrow p \pi^{-}$decay was observed as a $\mathrm{V}$ signature, a kinematical fit with seven overconstraints could be performed, otherwise there was only a single overconstraint. Due to these overconstraints in the kinematic fits very good resolutions of $1-3 \mathrm{MeV}$ were obtained for $\Lambda p$ invariant masses.

All these measurements find a sharp peak in the $\Lambda p$ invariant mass spectrum right at - or very close to - the $\Sigma N$ threshold, which in detail consists of two thresholds: the $\Sigma^{+} n$ threshold at $2.129 \mathrm{GeV}$ and the $\Sigma^{0} p$ threshold at $2.131 \mathrm{GeV}$. The observed peak has a width of only few $\mathrm{MeV}$ and is skew in shape with a very sharp rise at its low energy-side and a shallower fall-off towards higher energies - see Fig. 2.

In the measurement of Tan [163], which has the best energy resolution of $1 \mathrm{MeV}$, the high-energy fall-off appears as a kind of shoulder, so that the full observed structure can be fitted quantitatively by two Breit-Wigner resonant structures with mass and width $m_{1}=2128.7 \pm 0.2 \mathrm{MeV}, \Gamma_{1}=7.0 \pm 0.6$ $\mathrm{MeV}$ and $m_{2}=2138.8 \pm 0.7 \mathrm{MeV}, \Gamma_{2}=9.1 \pm 2.4 \mathrm{MeV}$.

It is tempting to associate the main structure with a $\Sigma N$ cusp effect due to the strong coupling between $\Lambda p$ and $\Sigma N$ channels. In Ref. [166] a phenomenological treatment of this scenario has been sketched by use of asymmetric Flatté [168] distributions, which assume an underlying $S$-wave resonance in the $\Lambda p$ channel. It has been shown that a satisfying description of the data in the region of the $\Sigma N$ threshold may be obtained, however, without being able to fix mass and width of the underlying hypothetical resonance in any satisfying manner.

Toker, Gal and Eisenberg [169] studied this scenario in the Faddeev formalism testing various $Y N$ interactions. They obtain a good description of the $\Lambda p$ invariant mass spectrum of Tan [163] without invoking a $\Sigma N$ bound state. According to their description the main peak may be regarded as a genuine three-body cusp, whereas the high-energy shoulder results from the interference between direct $\Lambda$ production processes and those involving the intermediate $\Sigma N-\Lambda N$ conversion.

Subsequent calculations by Torres, Dalitz and Deloff [170] in the Faddeev formalism, too, come to a similar though in detail somewhat different conclusion. According to their result the Tan data imply that there is no stable bound state - i.e. a pole in the second Riemann sheet of the complex energy plane of $Y N$ systems above the $\Sigma N$ threshold - in the coupled $\Sigma N-\Lambda N$ system, but the data require a virtual state, i.e. a pole in the fourth sheet near the $\Sigma N$ threshold. Similar conclusions have been reached in Ref. [171]. These conclusions support the view that this ${ }^{3} S_{1}$ state in the coupled $\Sigma N-\Lambda N$ system is simply the strange $S=-1$ analogue of the deuteron as envisaged by Oakes [72] in 1961, but with the twist that this deuteron counterpart appears as a virtual state.

With the advance of proton accelerators to deliver high-intensity beams at energies above the kaon production threshold the $p p \rightarrow \Lambda p K^{+}$reaction came into competition to the $K^{-} d \rightarrow \Lambda p \pi^{-}$reaction for the investigation of the $\Sigma N-\Lambda N$ system. After some early measurements at Brookhaven [172, 173] and Princeton [174] inclusive high-resolution measurements have been carried out at SATURN, Saclay utilizing the SPES4 magnetic spectrometer for the $K^{+}$detection [175]. The $\Sigma N$ cusp has been again observed in these measurements, though it appears only as an enhancement at the $\Sigma N$ threshold, since in this inclusive experiment the $\Sigma$ production can not be separated from the $\Lambda$ production. The observed enhancement due to the cusp effect agrees well with corresponding predictions of Deloff [176] and Laget[177]. Aside from the cusp related enhancement a small, narrow structure with a statistical significance of $3 \sigma$ has been observed in the Saclay measurements near $2010 \mathrm{MeV}$. It has been noted in Ref. [175] that this structure as well as the cusp effect coincide within $10 \mathrm{MeV}$ to the predictions of Aerts and Dover [83, 84 for the mass of $P$-wave the singlet and triplet states $D_{s}$ and $D_{t}$ having a 


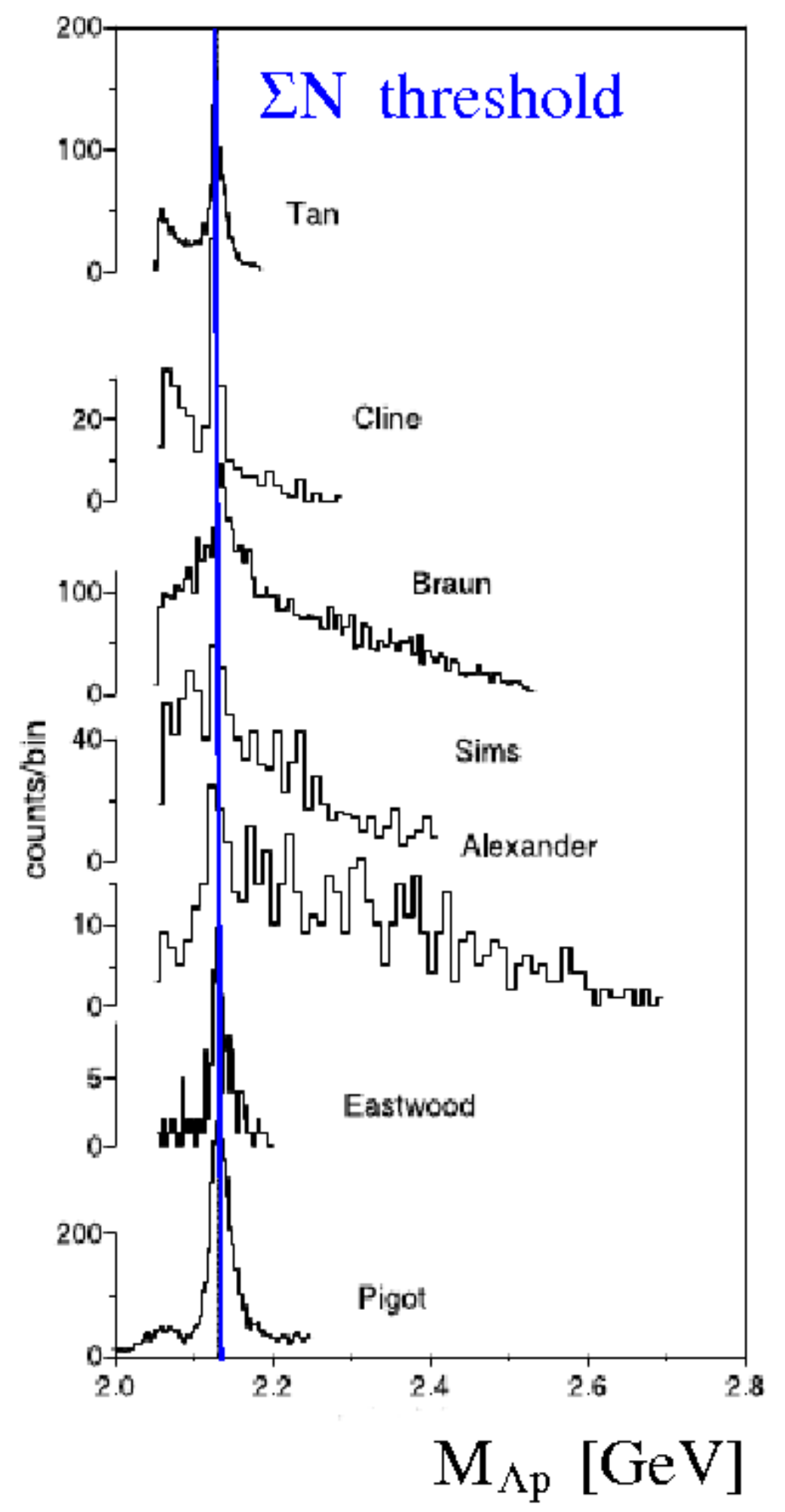

Figure 2: Distribution of the $\Lambda p$-invariant mass $M_{\Lambda p}$ as obtained from the $K^{-} d \rightarrow \Lambda p \pi^{-}$reaction studied in Refs. [163, 161, 166, 164, 162, 165, 167] (from top to bottom). The vertical line denotes the $\Sigma N$ threshold averaged over the masses of $\Sigma^{+} n$ and $\Sigma^{0} p$. From Ref. [180]. 
$q^{4}-q^{2}$ quark structure. Also the observed widths are in accord with the predictions.

In conclusion, whereas it is obviously not unambiguous, whether the cusp effect at the $\Sigma N$ threshold is correlated with a real or virtual dibaryon state, the nature of the narrow structure near $2010 \mathrm{MeV}$ can be clarified by an improved measurement with higher resolution and statistics. In fact, such an improved measurement has been carried out recently at COSY utilizing the BIG KARL magnetic spectrometer providing a missing mass resolution of $0.84 \mathrm{MeV}$ [178]. This measurements finds no evidence for a dibaryon state between 2058 - $2105 \mathrm{MeV}$ yielding very low upper limits in the range of nb/sr. In the same set of measurements also the cusp related enhancement at the $\Sigma N$ threshold has been confirmed 179 .

In a recent review by Machner et al. [180] all measurements in the $\Sigma N$ cusp region - including the more recent exclusive and kinematical complete COSY-TOF measurements, which will be discussed in section 8.2 - have been reevaluated in detail. In that review the conclusion is reached that after all

a two Breit-Wigner resonance ansatz provides the best overall description of the data with resonance parameters in agreement with those of Tan[163].

\subsection{Conclusions about this Period}

Animated by the enormous amount of predicted states, a worldwide rush of experimental dibaryon searches started in the eighties - ending finally with a vast number of claims. But unfortunately no single one survived critical experimental and analytical examinations.

Among the reasons for this striking failure was certainly the insufficient quality of data, be it lowstatistics bubble-chamber data or data from inclusive measurements, performed often by single-arm detectors. For critical, though also very amusing reviews of this epoch see, e.g., those given 1988 by K. K. Seth [5, 6], who pioneered this field by many high-quality measurements un-masking thus many of the dibaryon claims as statistical fluctuations or detector artifacts in poor data - see e.g. Fig. 22 in Ref. [5], where in a high-statistics and high-resolution $\vec{p} d \rightarrow p X$ measurement many of the dibaryons claims have been excluded in the mass range $1877-2200 \mathrm{MeV}$. In his conclusions he summarizes:

- "Nobody, anywhere, has seen a genuine, bona-fide, gold-(silver, nickel-, or even un-)plated dibaryon, yet!"

- "The days of doing quick and dirty (Q \& D) experiments are over."

- "The days of making Q \& D predictions are over."

- "The days of inventing dibaryons to explain the difference between poor experiment and poorer theory (or vice-versa) are over."

- "We must do honest hard work, or quit."

And he recommends for future research: "We should concentrate on exclusive experiments ...", a point, which we will pursue in the following.

\subsection{A Possible Remnant from this Period - a Broad Resonance structure around the $\Delta N$ Threshold}

Possibly, there exists a survivor from this era - though not a narrow, but broad resonance structure around the $\Delta N$ threshold. Already in the fifties it was observed [68, 69, 70] that the ${ }^{1} D_{2}$ partial wave of the $N N$ system indicates a resonant behavior in the $\pi^{+} d \rightarrow p p$ reaction corresponding to a resonance with $I\left(J^{P}\right)=1\left(2^{+}\right), \mathrm{m} \approx 2160 \mathrm{MeV}$ and $\Gamma \approx 120 \mathrm{MeV}$. Dyson \& Xuong identified this candidate with their asymptotic $N \Delta$ state $D_{12}$ to fix the remaining parameter B in their ansatz - see Table 1 . 
The possible existence of this resonance was confirmed in the sixties by Dick Arndt [181] analyzing $p p$ scattering data and calling this resonance structure a "Spin-2 Regge Recurrence of the ${ }^{1} S_{0} p-p$ Pole".

Later-on, in the eighties and nineties, the Gatchina group [182, 183, 184] and the SAID data analysis group [110, 111, 185, 186, 187, 188, 189, 190, could demonstrate by partial wave analyses based on highquality cross section and polarization data for $p p \rightarrow p p, \pi d \rightarrow \pi d$ and $\pi d \rightarrow p p$ reactions that the ${ }^{1} D_{2}$ partial wave exhibits a pronounced looping in the Argand diagram - see Fig. 3 - in favor of a true $s$-channel resonance. However, since the mass of the resonance is close to the $N \Delta$ threshold and since in addition the width of the resonance is compatible with that of the $\Delta$, it has been argued that the observed features represent a $N \Delta$ threshold phenomenon rather than a $s$-channel resonance and the observed looping in the Argand plot is merely a reflection of the usual $\Delta$ excitation in the presence of a nucleon with both being forced to be at rest relative to each other due to the threshold condition 4, 191, 192, 193, 194, 195, 196. In this discussion there have been many pros and cons, see, e.g. Refs. [4, 5, 193, 194, 195, 196, 197, 198, 199, 200]. In a series of papers [201, 202] Hoshizaki concluded that interpretations in terms of a $N \Delta$ virtual-state pole or a threshold cusp are invalid, rather it constitutes a true $S$-matrix pole at (2144 - i 55) MeV. A similar conclusion has been reached by Ueda et al. [203].

But still we are left with the problem that these values do not deviate significantly from what is expected for a conventional $\Delta$ excitation by $t$-channel meson exchange between the two nucleons.

We note in passing that in the SAID partial wave analyses also the ${ }^{3} P_{2}-{ }^{3} F_{2},{ }^{3} F_{3},{ }^{1} G_{4}$ and ${ }^{3} F_{4}-{ }^{3} H_{4}$ $N N$ partial waves exhibit a looping in the Argand diagram - see Fig. 3 , though their effect in the observables is small compared to the dominant ${ }^{1} D_{2}$ partial wave in the $\Delta N$ region. For the ${ }^{3} F_{3}$ partial wave a pole at $(2170$ - i 72) $\mathrm{MeV}$ has been found [190], which is right at the $\Delta N$ threshold. Hence it is also plausible that the deduced width is larger than that of the ${ }^{1} D_{2}$ resonance. In addition a pole have been located in the threshold region for the ${ }^{3} P_{2}-{ }^{3} F_{2}$ coupled partial waves at (2167 - i 75) MeV [190].

Garcilazo et al. used local [204] as well as non-local [205] $\Delta N$ and $\Delta \Delta$ potentials derived from the quark-cluster model to analyze the bound-state problem of the $\Delta N$ system for the case that the two-body system is in relative $S$-wave. As a result they find that the $\Delta N$ system has just a single bound state, which sits right at the $\Delta N$ threshold and which corresponds to the experimental finding of the resonant ${ }^{1} D_{2} N N$ partial wave. Since they restricted their studies to relative $S$-waves between $N$ and $\Delta$, they did not investigate the situation with regard to the ${ }^{3} F_{3} N N$ partial wave.

More recently the $N N \pi$ system was investigated by Gal and Garcilazo utilizing Faddeev calculations with hadronic interactions [35, 36]. They also find a resonance pole at (2147 - i 60) $\mathrm{MeV}$ for the $I\left(J^{P}\right)=1\left(2^{+}\right)$state.

A completely different approach for shedding more light onto the longstanding question, whether there are genuine dibaryon resonances in the $\Delta N$ region or not, has been presented very recently by Platonova and Kukulin [206]. They argue that the use of soft meson baryon form factors consistent with $\pi N$ elastic scattering gives too low cross sections in conventional $t$-channel meson and nucleon exchange calculations for single-pion production, in particular for the $p p \rightarrow d \pi^{+}$reaction. Only by inclusion of the formation and the decay of dibaryon resonances - in particular the $D_{12}$ resonance with $I\left(J^{P}\right)=1\left(2^{+}\right)$in the ${ }^{1} D_{2} N N$ partial wave - in the intermediate reaction process a proper description of the experimental data can be obtained. By additional inclusion of corresponding resonances in ${ }^{3} F_{3}$ and ${ }^{3} P_{2}$ partial waves even polarization observables can be quantitatively described for the first time [207.

We shall return to the $\Delta N$ threshold phenomena in sections 11.2 and 11.3. 

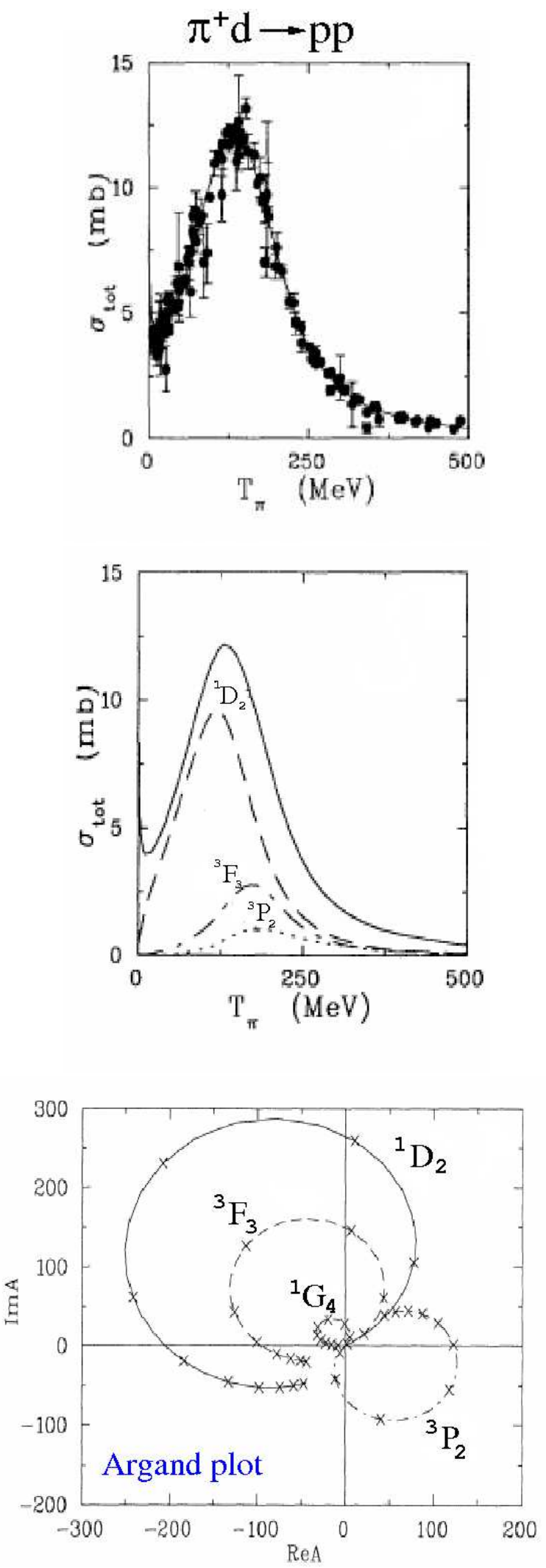

Figure 3: Energy dependence of the total cross section of the $\pi^{+} d \rightarrow p p$ reaction as obtained by measurements (top) and by the SAID partial-wave analysis (middle) together with its decomposition into its most prominent contributions from the $p p$ partial waves ${ }^{1} D_{2},{ }^{3} F_{3}$ and ${ }^{1} G_{4}$. Bottom: Argand plot for selected partial waves, which exhibit a pronounced looping. From Ref. [186]. 


\section{An Intermezzo in Nuclei: The Pionic Double Charge Ex- change Reaction}

In the pionic double charge exchange (DCX) reaction on nuclei $\pi^{+} A(Z, N) \rightarrow \pi^{-} B(Z+2, N-2)$ and $\pi^{-} A(Z, N) \rightarrow \pi^{+} B(Z-2, N+2)$, respectively - in nuclear physics convention denoted as $\mathrm{A}\left(\pi^{+}, \pi^{-}\right) \mathrm{B}$ and $\mathrm{A}\left(\pi^{-}, \pi^{+}\right) \mathrm{B}$, respectively - , two neutrons are converted into two protons or vice versa. This reaction ensures that the process is a genuine two-nucleon process depending heavily on the correlations between the two active nucleons. The cross sections have been found to be largest at low incident pion energies and in addition highly sensitive to short-range correlations there - for a review, see e.g. Refs. [208, 209]. This is particularly true for so-called nonanalog transitions, for which the isospins of initial and final nuclear states differ by two units. Hence it is not astonishing that this reaction has been considered to be well suited for the search of dibaryons.

Since secondary pion beams suffer from background due to pion decay, DCX measurements have first been carried out at high pion energies, where the effective pion lifetime in the laboratory system is larger and hence this background is smaller than at low energies. In the latter case most of the pions produced at the production target have already decayed before reaching the final target for the actual measurement.

Hence at the pion factories the first DCX measurements concentrated on pion energies in the region of the $\Delta$ excitation and also on so-called double isobaric analog transitions (DIAT) in nuclei. In these reactions the isospin in initial and final nuclear states stays identical, i.e. the two active neutrons in the nucleus are converted into two protons, which stay in the same nuclear orbit, thus possessing the same wave function in the final nucleus as the two original neutrons in the initial nucleus had before only the 3rd component of the isospin is changed. Hence the overlap between initial and final nuclear wave functions is maximal with the consequence that also the cross section should get comparatively large.

These DIAT measurements in the $\Delta$ region exhibited a diffraction pattern in the angular distributions, which can be accounted for in strong absorption model calculations, if an isotensor term is included [210]. The reaction process is assumed to be a two-step process of two sequential single charge exchanges in the nucleus. In this conception the incoming positive pion gets absorbed by a valence neutron leading to a (virtual) $\Delta^{+}$excitation, which deexcites into a proton in the same orbit and a (virtual) neutral pion. The latter propagates to another neutron, preferentially in the same orbit, where it gets absorbed by exciting this neutron to a (virtual) $\Delta^{0}$ state, which again deexcites into a proton in the same orbit and a $\pi^{-}$particle, which is then emitted from the final nucleus.

The measured dependence of the DIATs on the mass number A of the investigated nuclei is in agreement with this two-step process in the strong absorption limit. In this picture each step of single charge exchange proceeds with $A^{-1}$ in the amplitude, since the charge of a pion is exchanged on a specific nucleon out of $A$ nucleons. Due to strong absorption (black disc) the reaction volume is restricted to the circumference of a disc providing another factor $A^{1 / 3}$ in the amplitude. Hence in total we expect a $A$ dependence of $A^{-1} A^{-1} A^{1 / 3}=A^{-5 / 3}$ in the amplitude and of $A^{-10 / 3}$ in the cross section, respectively - and this is what actually has been observed for the DIAT measurements in the $\Delta$ resonance region.

At incident pion energies above the $\Delta$ resonance region the measured cross sections get somewhat larger - as expected from the reduced pion absorption in nuclei, but basically the two-step concept for DIATs proved to stay valid there, too.

Having understood the reaction mechanism for DIATs in the $\Delta$ resonance region and above it came as a surprise that the first measurements at low incident pion energies showed the cross sections to be largest in the region of $T_{\pi} \approx 50 \mathrm{MeV}$. In contrast to this experimental finding two-step models had predicted cross sections to be smaller by an order of magnitude - among others due to the fact that the forward-angle single-charge exchange cross section vanishes because of the well-known destructive 
interference between $s$ - and $p$-waves in the $\pi N$ system.

In this situation G. Miller [211] proposed six-quark cluster components of nuclear wave functions as an explanation for the unexpected large DIAT cross section. In this scenario a dibaryonic six-cluster would be formed as an intermediate state. Since the pion-absorption operator is an axial vector and due to spatial symmetry there are only two possible intermediate states with $I\left(J^{P}\right)=0\left(1^{+}\right)$and $2\left(1^{+}\right)$. Such dibaryon states had been predicted by Mulders and Thomas [79] with masses of 2180 and 2460 $\mathrm{MeV}$, respectively. Though Miller's calculations gave a reasonable description for the forward angle data for the DIAT in ${ }^{14} \mathrm{C}$ - in particular, if pion absorption is taken into account [212] - see, e.g., Fig 4.18 in Ref. [208], conventional calculations including isotensor terms subsequently turned out to be at least as successful or even more successful [213] - see Fig. 4.19 in Ref. [208]. Later-on G. Miller [214] pointed out that the six-quark cluster assumption also necessitates a resonance-like energy dependence in the total DIAT cross section on ${ }^{14} \mathrm{C}$, which should peak at $T_{\pi} \approx 425 \mathrm{MeV}$, which again was not observed 215] - see, e.g. Fig. 4.28 in Ref. [208]. Among others a reason for this failure could have been that the two-step process of a DIAT not only depends on short-range correlations, but also on longer-range correlations, which possibly blur the six-quark cluster contribution.

On the contrary, short-range correlations play a central role in non-analog transitions, which mostly have been measured as a transition between ground states, e.g. ${ }^{12} \mathrm{C}\left(\pi^{+}, \pi^{-}\right){ }^{12} \mathrm{O}$. In fact, initially the cross sections of these non-analog transitions were massively underestimated. Since no simple twostep picture was available for this process, it were thought to have tiny cross sections. However, first measurements in the $\Delta$ resonance region revealed these cross sections to be of the same order of magnitude as the DIATs and partly nearly as large as those. Also unexpectedly, the observed dependence on the nuclear mass number $\mathrm{A}$ was not that of a two-step process, namely proportional to $A^{-10 / 3}$, but that of an effective one-step process being proportional to $A^{-4 / 3}$. The latter can be understood, if the two active neutrons are exchanged into two protons in a single step, so that in the strong absorption limit we have a DCX amplitude being proportional to $A^{-1} A^{1 / 3}=A^{-2 / 3}$.

Another unexpected feature of the non-analog transitions observed in the $\Delta$ resonance region and above was the energy dependence of the forward-angle cross sections, which revealed a resonance-like structure reminiscent of a $\Delta$ excitation in nuclei - see Fig. 4. In general, the peak was found to be just around the $\Delta$ mass, whereas the width of the observed structure was somewhat smaller in the range of $70-90 \mathrm{MeV}$ [216].

To accommodate these unexpected features in an appropriate model description, the so-called $\Delta N$ interaction (DINT) model has been designed [217, 218, where - as in the DIAT process - the incoming $\pi^{+}$particle initially excites a neutron to $\Delta^{+}$. However, the subsequent $\Delta N$ interaction does not lead to the exchange of a $\pi^{0}$ with the neighboring neutron, but to the exchange of a $\pi^{+}$with the consequence that the neighboring neutron is charge exchanged to a proton, whereas simultaneously the $\Delta^{+}$is charge exchanged to $\Delta^{0}$, which subsequently decays into proton and emitted $\pi^{-}$particle. Since this effective single-step process certainly is of very short range, it has been proposed [219, 220] that the DINT process may involve six-quark clusters in the intermediate state. Though this is similar to Miller's proposal, it is now explicitly specified only for the short-range DINT process. In addition Johnson and Kisslinger [219] showed that the contribution of the intermediate $I\left(J^{P}\right)=0\left(1^{+}\right)$state is small compared to that of the $I\left(J^{P}\right)=2\left(1^{+}\right)$state.

From the comparison to the experimental results for non-analog transitions it follows that the dominating intermediate state, which gives rise to the $\Delta$-like resonance structure in the energy-dependence of non-analog transitions, does not correspond to a mass of $2460 \mathrm{MeV}$ as predicted by Mulders and Thomas [79] and adopted by Miller, but to a mass of 2140 - $2170 \mathrm{MeV}$, i.e, a mass corresponding closely to the $\Delta N$ mass. That way it would rather represent the $D_{21}$ dibaryonic state predicted to be at the same mass as $D_{12}$ by Dyson and Xuong [33] - see sections 3.2 and 11.2.

An even more exciting feature of non-analog transitions has been revealed by subsequent measurements [221, 222, 223, 224, 225, 226, 227] at low energies. There these transitions exhibit in general not 
only larger cross sections than at higher energies, but also a very pronounced narrow resonant structure in the energy dependence (Fig. 4), which we will discuss in detail in the following.

\subsection{Yet Another Dibaryon Candidate: $d^{\prime}(2065)$}

On the basis of a QCD string model Schepkin and coworkers at ITEP, Moscow, predicted the lowestlying dibaryon states to be a triplet of isoscalar states with $J^{P}=0^{-}, 1^{-}$and $2^{-}$having an $\mathrm{l}=1$ orbitally excited $q^{4}-q^{2}$ structure. This triplet corresponds to the triplet predicted by the Nijmegen group on the basis of the MIT bag model, however at somewhat smaller masses, in the range $2050-2140 \mathrm{MeV}$ [88, 90]. Since the $0^{-}$and $2^{-}$members of the triplet cannot couple to the $N N$ system due to their quantum numbers, the only possible hadronic decay channel is $N N \pi$. In consequence the decay width should be very small in the range of only few $\mathrm{MeV}$ or even below. Due to spin-orbit splitting the $2^{-}$ state was expected to be the lowest-lying state.

Since the predicted mass range corresponded just to the bump structure seen around $T_{\pi} \approx 50$ $\mathrm{MeV}$ in the DIATs on ${ }^{14} \mathrm{C}$ and also ${ }^{18} \mathrm{O}$, Martemyanov and Schepkin proposed the $2^{-}$state to be the reason for this observed structure [89]. Indeed, in subsequent work [223, 228, 224, 225, 226, 227] it could be demonstrated that all low-energy data on DIATs and non-analog transitions in nuclei ranging from ${ }^{7} \mathrm{Li}$ to ${ }^{93} \mathrm{Nb}$ could be quantitatively described by the assumption of the excitation of a narrow dibaryon resonance in the intermediate state: the $d^{\prime}(2065)$. Only, the experimental angular distributions requested a spin-parity assignment of $0^{-}$instead of the previously assumed $2^{-}$assignment.

In the description of the data the dibaryon resonance effect has been assumed to sit upon a background of non-resonant conventional processes. In case of DIATs these conventional processes have been described by the model of Auerbach et al. (AGGK), where the two-step process is calculated within the seniority concept for the nuclear structure aspects [229, 230]. The exceptional role of the DIAT on ${ }^{48} \mathrm{Ca}$, which constitutes the only case, where no bumpy energy dependence at low pion energies was observed, has been successfully explained by this model. However, for all other cases this model largely underestimated the measured cross sections. In particular, it could not explain the observed steep resonance-like energy dependence there. This failure had been common to other theoretical investigations at that time.

For the non-analog transitions, which exhibit the resonance-like structure with a width of only about $20 \mathrm{MeV}$ even much more pronounced, no adequate conventional description had been available, since the AGGK model predicted vanishing cross sections in general. The DINT mechanism, which was the only quasi single-step process to describe the data at higher energies, also provided only tiny cross sections, since at $T_{\pi} \approx 50 \mathrm{MeV}$ solely the low-energy tail of the $\Delta$ excitation is left. Hence essentially the full measured cross section was attributed to the $d^{\prime}$ resonance excitation.

A consistent successful description of all low-energy DCX data, both DIATs and non-analog transitions, was achieved with the $d^{\prime}$ model by adjustment of just the resonance parameters for mass and width resulting in a mass of $2065 \pm 5 \mathrm{MeV}$, a decay width into the $N N \pi$ system of $0.5 \mathrm{MeV}$ and a spreading width due to the nuclear surrounding of $10-20 \mathrm{MeV}$ [227]. The further broadening of the resonance due to the Fermi motion of the active nucleon pair was calculated microscopically. It should be noted that in initial calculations a spreading width of only $5 \mathrm{MeV}$ was assumed, which gave too large $d^{\prime}$ contributions.

The success of the $d^{\prime}$ hypothesis to describe the low-energy DCX data initiated a number of theoretical investigations for such a dibaryon [241, 242, 243, 244, 245, 246, 203, 247, 249, 250, 251, 252, 253, 254. The Tübingen theory group [241, 242, 243, 244, 245, 246] calculated a possible dibaryon with the quantum numbers $I\left(J^{P}\right)=0\left(0^{-}\right)$of $d^{\prime}$ in constituent quark, quark shell-model, colored quark cluster and quark pair creation models. As a common result the mass of such a dibaryon was found to be in the range of $2300-2400 \mathrm{MeV}$, if the confinement parameters obtained from the baryon spectrum were used. Only if the confinement was strongly weakened and thus the size of such a dibaryon was enlarged, a 


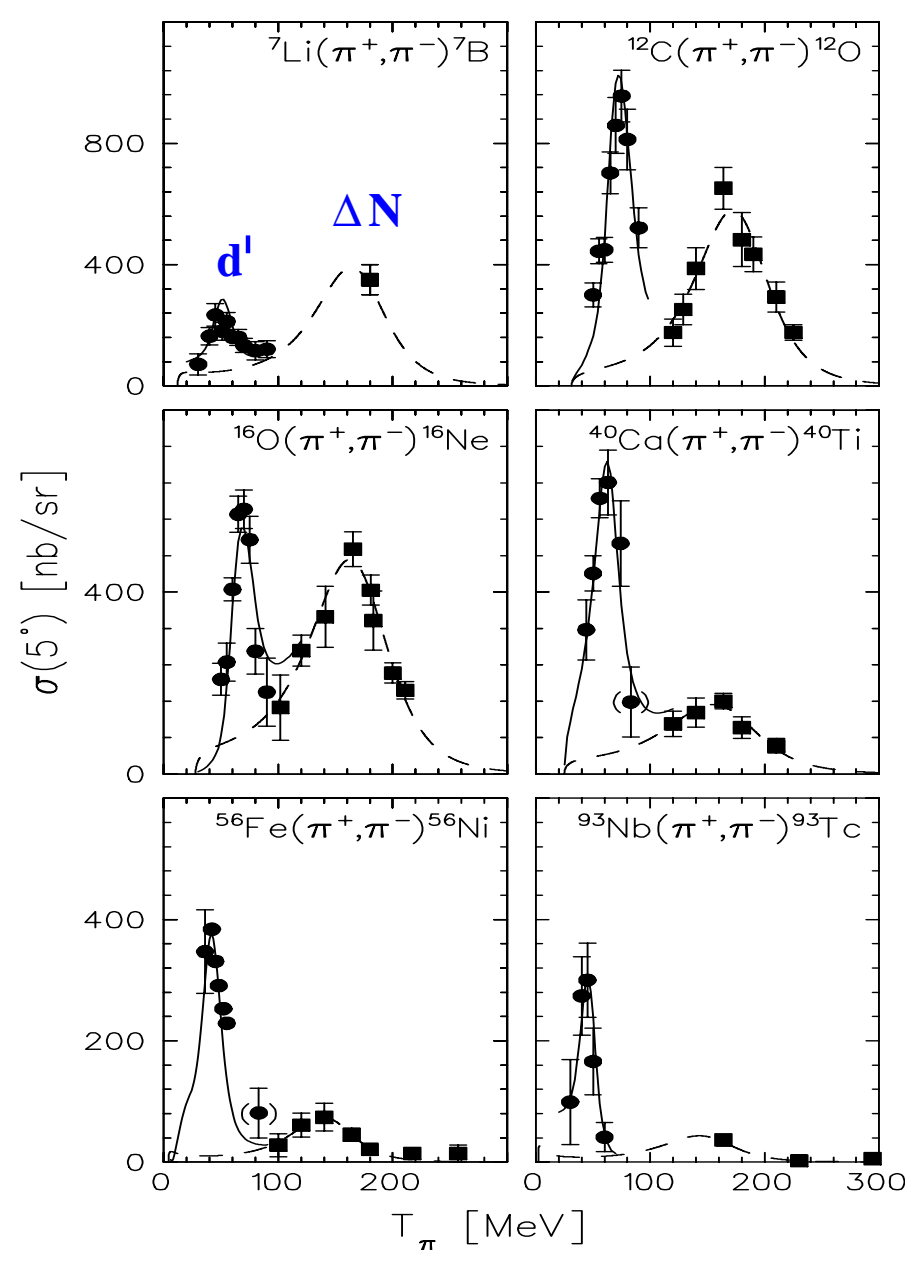

Figure 4: Energy dependence of the forward-angle cross sections for nonanalog DCX groundstate transitions. The solid lines show calculations assuming the formation of the hypothetical $N N \pi$ resonance $d^{\prime}$ interfering with the DINT mechanism forming a resonating $\Delta N$ system. From Refs. [227, 240]. 
mass as well as a decay width as low as needed for the explanation of the DCX data was obtained.

Ueda calculated such a dibaryon solely by use of $\pi N N$ dynamics. Initially he obtained a mass in the range 2060 - $2090 \mathrm{MeV}$ [247], but in an improved calculation he obtained a mass of only $4-5 \mathrm{MeV}$ above the $N N \pi$ threshold, i.e around $2022 \mathrm{MeV}$ [248]. Essentially the same conclusion was reached by Garcilazo solving nonrelativistic Faddeev equations with hadronic interactions [249]. In further studies within the framework of $\Delta N$ and quark cluster models it was concluded that $d^{\prime}$ should have isospin $I=2$ rather than $I=0$ [250, 252, 253], which also is compatible with the experimental observations. It also was shown that such a dibaryon resonance will increase its width in the nuclear medium by an order of magnitude - in agreement with experiment - but change its mass by only a few MeV.

A $J^{P}=0^{-}$dibaryon resonance was also looked at in the Skyrme model and it was demonstrated that this model leads to such a low-lying state only, if the isospin is $I=2$ [254] - in agreement with the conclusion reached by Garcilazo et.al..

A crucial disadvantage of using the DCX reaction for the dibaryon issue is that it can be conducted only on nuclei, but not on the basic $n n$ system, since the latter is unbound and hence not available as a target. Therefore any theoretical comparison with the data suffers from the fact that the nuclear structure in initial and final states is not fully under control. The substantial nuclear structure dependence of DCX calculations for DIATs and also non-analog transitions has been demonstrated impressively in Refs. [236, 237], where it has been shown that uncertainties in the nuclear wavefunctions can easily lead to a factor of two uncertainty in the predicted cross sections - in addition to pion wave distortion, which at least partially is the cause for the rapid fall-off of the cross section beyond $T_{\pi}=50 \mathrm{MeV}$. Taking all this into account, a number DIATs and non-analog transitions can be accounted for at least qualitatively by conventional calculations, though the data remain still much better reproduced by the $d^{\prime}$ resonance assumption.

The lightest target nucleus for observing the DCX process in a binary reaction to a discrete final state is ${ }^{7} \mathrm{Li}$. For lighter nuclei the DCX process leads only to the unbound nuclear continuum. With regard to $d^{\prime}$ this means that it no longer can be formed off-shell in the presence of $A-2$ nucleons, but only produced on-shell associatedly with the simultaneous release of spectator nucleons into the continuum - with the consequence that the $d^{\prime}$ signature in this now inclusive DCX measurements will be much less pronounced. Nevertheless, detailed DCX continuum measurements have been carried out on ${ }^{3} \mathrm{He}$ [231] and ${ }^{4} \mathrm{He}$ [232, 233, 234], but the results finally were inconclusive on the question of $d^{\prime}$, since it turned out that the initially assumed small spreading width was strongly underestimated [235]. With the more realistic spreading width the $d^{\prime}$ effect in these measurements got too small to be uniquely identified.

Finally, also in this case the existence of a narrow dibaryon resonance could not be established unambiguously and nature might have bluffed us again. Though the description of the data by inclusion of the as of yet hypothetical $d^{\prime}$ resonance works amazingly well, it cannot be excluded that by the complexity of nuclear structure and pion-nucleus interaction the observed resonance structure may finally find its explanation in a capricious composition of diverse conventional effects.

A possibility to search for $d^{\prime}$ in a basic reaction is neutral pion photoproduction from the deuteron, which has been discussed already above in section 4.2.3. However, the estimated $d^{\prime}$ production cross section in this reaction is in the order of $0.5-1 \mu \mathrm{b}$ compared to a background from conventional processes, which is larger by nearly two orders of magnitude [238]. A high-resolution measurement [139] of the $\gamma d \rightarrow \pi^{0} X$ reaction at MAMI found no statistically significant signal from a narrow dibaryon resonance at masses below $2100 \mathrm{MeV}$. But the deduced upper limits were still an order of magnitude larger than the predicted $d^{\prime}$ signal, so that also this measurement could not provide an answer, whether $d^{\prime}$ really exists.

Also electro-production of $d^{\prime}$ has been looked at by using ARGUS data on $\gamma^{* 16} O \rightarrow p p \pi^{ \pm} X$. Though the invariant $p p \pi^{+}$and $p p \pi^{-}$spectra were in agreement with the $d^{\prime}$ hypothesis, no firm conclusion could be drawn due to low statistics [255]. 
More promising has been the prediction of a $d^{\prime}$ signal in another basic reaction, the $p p \rightarrow p p \pi^{+} \pi^{-}$ reaction, where the $d^{\prime}$ cross section has been estimated to be only one order of magnitude below the cross section for conventional processes [239]. We will discuss the results from corresponding measurements in section 8.3.

\section{More Recent Searches for Strange $(S=-1)$ Dibaryons}

Recently the quest for strange dibaryons experienced a revival, when Akaishi and Yamazaki [256, 257, 258 predicted deeply bound antikaonic nuclear systems. It was argued that if the attractive $\bar{K} N$ interaction is strong enough to form $\bar{K}$-nuclear sytems with binding energies such large that they reside below the $\bar{K} N \rightarrow \pi \Sigma$ threshold, then such states will have a very narrow width and constitute very compact objects. In fact, subsequent measurements at KEK with $K^{-}$ions stopped on ${ }^{4} \mathrm{He}$ indicated such deeply bound structures [259]. However, an improved follow-up experiment [260] did not confirm the previous findings.

In the context of deeply bound antikaonic nuclear states also the basic system $p p K^{-}$was investigated both theoretically and experimentally. Initial calculations predicted a deeply nuclear-bound quasistable state with $I\left(J^{P}\right)=\frac{1}{2}\left(0^{-}\right)$, a binding of about $50-80 \mathrm{MeV}$ and a decay width into $\Sigma p \pi$ in the order of $60-110 \mathrm{MeV}$ [258, 261, 262, 263], whereas more sophisticated recent calculations give very moderate binding energies in the range of $10-20 \mathrm{MeV}$ and widths in the order of $40-70 \mathrm{MeV}$ [264, 265, 266]. For a recent review of this state, which asymptotically can be viewed also as a bound $\Lambda(1405) N$ dibaryon, see Ref. [267]. Note that $\Lambda(1405)$ itself is likely to be a meson-nucleon molecular state as derived by recent lattice QCD [268] and chiral $S U(3)$ calculations [269].

In initial FINUDA measurements [270], where $K^{-}$ions were stopped on ${ }^{6} \mathrm{Li},{ }^{7} \mathrm{Li}$ and ${ }^{12} \mathrm{C}$ targets, a $70 \mathrm{MeV}$ wide structure was found in the $\Lambda p$ invariant-mass spectrum. From the observed back-toback angular correlation it was concluded that this structure corresponded to an intermediate $p p K^{-}$ system bound by as much as $115 \mathrm{MeV}$. However, this interpretation was later-on heavily criticised [271, 272, 273] arguing that the observed spectrum may be well explained by final-state interaction of the produced $\Lambda p$ pair with the residual nucleus.

In order to avoid complications due to a nuclear surrounding, the search for a $p p K^{-}$bound state subsequently concentrated on basic reactions, which contained only two baryons and where the dibaryon system in question was produced associatedly. Note that no formation reaction is possible in this case, because there is no $p p$ target.

Since the predicted $p p K^{-}$bound state can decay also into $\Lambda p$, the $p p \rightarrow \Lambda p K^{+}$raction was already proposed in Ref. 258] as a reaction well-suited to search for this predicted state. An subsequent analysis 274] of DISTO data on the $p p \rightarrow \Lambda p K^{+}$reaction at $T_{p}=2.85 \mathrm{GeV}$ claimed the observation of a compact $p p K^{-}$system at $2265 \mathrm{MeV}$ corresponding to a binding energy of $103 \mathrm{MeV}$ and a width of $118 \mathrm{MeV}$. The DISTO data revealed the corresponding broad structure in the $\Lambda p$ invariant-mass spectrum only after severe cuts and assuming pure phase-space distribution for the non-resonant background. The latter is at variance with the information from many preceding investigations that this reaction is strongly affected by $N^{*}$ excitations. Their reflections may easily produce a bump -like structure in the $\Lambda p$ invariant-mass spectrum. In fact, a recent reanalysis [275] of all published $\Lambda p$ invariant-mass spectra finds no hint for the claimed resonance with deduced upper limits, which are partly substantially smaller than the predicted cross section values. In contrast to this finding the claimed resonance structure corresponded to a cross section four times larger than the predicted one and exceeded even the $\Lambda(1405)$ production, which was thought to be part of the intermediate state.

A recent search for the $p p K^{-}$bound state via the $\gamma d \rightarrow K^{+} \pi^{-} X$ reaction at LEPS at Spring-8 also found no evidence for such a state, only upper limits [276].

Surprisingly, a very recent experiment at J-PARC claims evidence for a $p p K^{-}$-like structure in the 
measurement of the $\pi^{+} d \rightarrow K^{+} X$ reaction at $p_{\pi}=1.69 \mathrm{GeV} / \mathrm{c}$ [277]. The measured $K^{+}$missing-mass spectrum shows three prominent peaks corresponding to the quasifree production of $\Lambda, \Sigma$ and excited hyperons $Y^{*}=(\Sigma(1385), \Lambda(1405))$. The simulated spectrum deviates in shape from the measured one only at two positions: at the position of the well-known $\Sigma N$ cusp - see sections 4.2 .5 and 8.2 - and in the region of the $Y^{*}$ excitations, where the measured spectrum appears to be shifted to lower $K^{+}$ missing masses. If now in addition an emitted proton is measured in coincidence with the emitted $K^{+}$, then the quasifree production is heavily suppressed, since the Fermi momentum of a spectator proton originating from the target deuteron is very small and hence will not reach the proton detectors. As a result, the $\mathrm{K}^{+}$missing-mass spectrum with the condition of a coincident fast proton shows no longer the quasifree peaks, but only the well-known $\Sigma N$ cusp followed by some broad tail-like strength at higher missing masses - see Fig. 2b in Ref. [277]. If in a next step the thus obtained spectrum is divided by the original $K^{+}$missing mass spectrum without the coincidence condition - the so-called "coincidence probablity" spectrum -, then a pronounced broad bump structure arises around $2.3 \mathrm{GeV}-$ see Fig. 2c in Ref. [277]. A Breit-Wigner fit to this bump results in a resonance mass of $2275 \mathrm{MeV}$ and a width of $162 \mathrm{MeV}$. This structure also persists, if a coincidence with two fast protons is requested, though the data have now large statistical uncertainties. Note that this particular structure appears only after division by the inclusive spectrum, which is dominated by quasifree processes - a procedure, which is not easily understandable from a physics point of view.

As pointed out by A. Gal [9], a mass for a $p p K^{-}$bound state of $2275 \mathrm{MeV}$, which corresponds to a binding energy of about $100 \mathrm{MeV}$ with respect to the $p p K^{-}$threshold, is unacceptable theoretically. However, it might correspond to a meson-assisted dibaryon state with $I\left(J^{P}\right)=\frac{3}{2}\left(2^{+}\right)$and strangeness $S=-1$ predicted by Garcilazo and Gal in relativistic three-body Faddeev calculations for the $\pi \Lambda N-\pi \Sigma N$ coupled system [278]. This predicted $\Lambda N \pi$ resonance, which may be viewed as a ${ }^{5} S_{2}$ $\Sigma(1385) N-\Delta Y$ quasibound state is according to Gal and Garcilazo the lowest-lying $S$ - wave dibaryon with strangeness $S=-1$.

The mass of this dibaryon state is predicted to be $10-20 \mathrm{MeV}$ below the $\Sigma N \pi$ threshold, i.e. in just in the mass region of the structure found at KEK [277]. Whether there is indeed a connection, has to be clarified by further exclusive and kinematically complete experiments, which - in case of a real resonance structure - must be able to also extract its quantum numbers. It should be noted that a recent search by HADES [279] at GSI in the $p p \rightarrow \Sigma^{+} p K^{0}$ reaction finds no evidence for the predicted $I\left(J^{P}\right)=\frac{3}{2}\left(2^{+}\right)$state.

New light into this matter has been shed by a recent measurement of the ${ }^{3} H e\left(K^{-}, \Lambda p\right) n$ reaction at J-PARC, where $\Lambda$ and proton have been detected, whereas the emitted neutron has been identified by its missing mass [280]. These measurements, which currently are being updated by new data [281], exhibit a pronounced bump structure in the $\Lambda p$ invariant spectrum in the region of the $\Sigma p \pi, \Lambda(1405) p$ and $p p K^{-}$thresholds. A Breit-Wigner fit to this structure results in a mass of about $2355 \mathrm{MeV}$ and a width of about $110 \mathrm{MeV}$. This mass is several $\mathrm{MeV}$ above the $\Lambda(1405) p$ threshold and $16 \mathrm{MeV}$ below the $p p K^{-}$threshold. In a model description Sekihara, Oset and Ramos [282] interpret the data by a two-bump structure due to a quasi-elastic peak for $K^{-}$production in the first collision of the reaction and a peak associated to the production of a $p p K^{-}$quasi-bound state that decays into the $\Lambda p$ system. The calculated quasi-bound state has a binding energy of about $20 \mathrm{MeV}$ relative to $p p K^{-}$threshold and a width of about $80 \mathrm{MeV}$.

In conclusion, with the possible exception of the currently much discussed threshold structure near the $p p K^{-}$mass a convincing evidence for a dibaryon with strangeness $S=-1$ has not yet been found experimentally. 


\section{$7 \quad$ Status on the $H$ Dibaryon $(S=-2)$}

As mentioned already in section 4 the $H$ dibaryon was introduced 1977 by Jaffe [16] as a deeply bound $\Lambda \Lambda$ system with quark structure $u$ uddss and $I\left(J^{P}\right)=0\left(0^{+}\right)$. Calculating its mass by use of the attractive short-ranged color-magnetic interaction between quark-pairs in $S U(3)$-flavor symmetry he obtained a binding energy of some $80 \mathrm{MeV}$ relative to the $\Lambda \Lambda$ threshold, i.e. it would be stable with respect to a strong decay. Subsequently there have been numerous theoretical model calculations within various models, e.g., the MIT bag model [16, 75, 283], the constituent quark model [284], potential models [285], the Skyrmion model [286, 287, 288], the hybrid quark-cluster model [92, 289], the color-dielectric model [290] and the instanton model [291]. The predicted masses range from very deeply bound- much more than in Jaffe's prediction - to unbound, i.e. to masses above the $\Lambda \Lambda$ threshold. In particular, accounting for $S U(3)$-flavor symmetry breaking lead to a drastic reduction of the resulting attraction [283, 284, 285].

Very recently the question, whether the $H$ dibaryon is bound or not, received renewed attention, when two state-of-the-art lattice QCD calculations [12, 14] obtained the $H$ dibaryon to be bound by about $8 \mathrm{MeV}$ - albeit for values of the pion mass, which are still large compared to the real pion mass and without paying attention to hadronic thresholds. Subsequent theoretical investigations show that such predictions require still quite some fine-tuning [13] and that the mass also could well be between $\Lambda \Lambda$ and $\Xi N$ thresholds [15, 292].

A study within the chiral effective field theory [293] arrives at still smaller binding energies, if bound at all, and demonstrates that $S U(3)$ breaking effects induced by the differences of the pertinent two-baryon thresholds have a very pronounced impact that need to be properly incorporated in lattice QCD in order to produce realistic results. It is also pointed out that, if the $H$ dibaryon is bound, the dominant component should be $\Xi N$ rather than $\Lambda \Lambda$.

For an experimental search for the $H$ dibaryon, the possible decays of such an object have to be considered. If the $H$ dibaryon is bound with respect to the $\Lambda \Lambda$ threshold, it can only decay mediated by the weak interaction and the dominant decay route will be $H \rightarrow \Lambda N \pi$. In this case a very narrow resonance structure is expected in this decay channel. If the $H$ mass is above the $\Lambda \Lambda$ threshold, then the $H$ will decay predominantly hadronically via $H \rightarrow \Lambda \Lambda$. Such an unhindered fall-apart decay necessarily will cause the width of such an $H$ dibaryon to be very broad. The $H$ dibaryon resonance will get still broader, if its mass is even above the $\Xi N$ threshold. As pointed out already above, such broad resonances are very hard to identify uniquely in experiments, since they can hardly be distinguished from conventional non-resonant background processes. As demonstrated in section 4.4 regarding the broad resonance structure at the $\Delta N$ threshold, only detailed partial-wave analyses of scattering and formation reaction data are able to reveal a real resonance in such a case - a situation, which is not feasible in case of the $H$ dibaryon resonance.

There have been numerous experiments searching for a $H$ dibaryon. A deeply bound $H$ dibaryon has been excluded by the so-called "NAGARA" event, which exhibits the unambiguous signature of the double- $\Lambda$ hypernucleus ${ }_{\Lambda \Lambda}^{6}$ He produced via $\Xi^{-}$capture in emulsion [17, 18. If the mass $m_{H}$ of the $H$ dibaryon would be less than twice the $\Lambda$ mass in the nucleus, then the two $\Lambda$ s would be expected to form a $H$ dibaryon. Hence the existence of a double- $\Lambda$ hypernucleus with a $\Lambda-\Lambda$ binding energy $B_{\Lambda \Lambda}$ ( in the nucleus) leads to a lower limit for the mass of the $H$ dibaryon according to

$$
m_{H}>2 m_{\Lambda}-B_{\Lambda \Lambda}
$$

From the double- $\Lambda$ hypernuclei measurements, in particular the NAGARA event $B_{\Lambda \Lambda}=6.93 \pm 0.16 \mathrm{MeV}$ is obtained [18, which gives a lower limit of $7 \mathrm{MeV}$ for the $H$ binding energy and of $2224 \mathrm{MeV}$ for its mass, respectively, at the $90 \%$ confidence level.

The E224 experiment [19] at the KEK proton synchrotron searched for $H$ in the $\Lambda \Lambda$ production in the inclusive reaction $K^{-12} C \rightarrow K^{+} \Lambda \Lambda X$ and noted an enhancement in the $\Lambda \Lambda$ invariant-mass spectrum 
right at the $\Lambda \Lambda$ threshold. A follow-up measurement E522 [20] with improved statistics confirmed this findings, but could also demonstrate that the conventional $\Lambda \Lambda$ final-state interaction can quantitatively describe the observed threshold enhancement without any need for assuming a $H$ dibaryon resonance.

At BELLE a high-statistics search for $H$ dibaryon production was performed recently in inclusive $\Upsilon(1 s)$ and $\Upsilon(2 s)$ decays [21]. No indication of an $H$ dibaryon with a mass in the range from $35 \mathrm{MeV}$ below $2 m_{\Lambda}$ to $25 \mathrm{MeV}$ above $2 m_{\Lambda}$ has been observed in either the $H \rightarrow \Lambda \Lambda$ or $H \rightarrow \Lambda p \pi^{-}$decay channels. Stringent upper limits for the branching-fractions have been extracted, which are between one and two orders below that for inclusive $\Upsilon(1 s)$ and $\Upsilon(2 s)$ decays to antideuterons. Since deacys of $\Upsilon(1 s)$ and $\Upsilon(2 s)$ produce $S U(3)$ flavor-symmetric decays, these results put stringent constraints on the dynamical properties of the $H$ dibaryon. I.e., it must have dynamic properties very different from the deuteron or, if $M_{H}<2 m_{\Lambda}$, a strongly suppressed decay mode $H \rightarrow \Lambda p \pi^{-}$.

In heavy-ion collisons hyperons are produced in large numbers, which gives access to the study of the $\Lambda \Lambda$ interaction and search for the $H$ dibaryon. In measurements of $\mathrm{Au}-\mathrm{Au}$ collisons at $200 \mathrm{GeV}$ at RHIC by use of the STAR detector the $\Lambda \Lambda$ correlation function has been determined [23] giving access also to scattering length $a_{\Lambda \Lambda}$ and effective range $r_{\Lambda \Lambda}$ of the $\Lambda \Lambda$ interaction. Whereas in the original paper a slightly repulsive interaction has been derived, an improved analysis [24] arrives at a slightly attractive interaction with $-1.2 \mathrm{fm}<a_{\Lambda \Lambda}<-0.5 \mathrm{fm}$ and $3.5 \mathrm{fm}<r_{\Lambda \Lambda}<7 \mathrm{fm}$, if the $\Lambda$ samples do not include the feed-down contributions from long-lived particles. A negative scattering length excludes the existence of a bound state in the $\Lambda \Lambda$ system. However, it is found that the feed-down correction for $\Sigma^{0}$ decay reduces the sensitivity of the correlation function to the details of the $\Lambda \Lambda$ interaction such strongly that only the weaker constraint $a_{\Lambda \Lambda}>-1.2 \mathrm{fm}$ is left - which unfortunately does not completely exclude the existence of a bound state. For a more detailed discussion see also Ref. [25].

ALICE has searched for a weakly decaying $H$ dibaryon in central $\mathrm{Pb}-\mathrm{Pb}$ collisions at LHC [22] by looking for the decay mode $H \rightarrow \Lambda p \pi^{-}$. To this end a $\Lambda$ has to be identified by two tracks belonging to a proton and a pion and originating from a secondary vertex. In addition another decay pattern reconstructed from a proton and a pion is required to be found at the decay vertex of the assumed $H$ dibaryon. Since the considered weak decay will only have a sizeable branching, if the $H$ dibaryon is bound and hence can not decay hadronically into $\Lambda \Lambda$, this experiment is sensitive only for the bound case. No evidence has been found for a bound $H$ dibaryon yielding upper limits for the mass range 2200 - $2231 \mathrm{MeV}$, i.e. bindings energies smaller than $31 \mathrm{MeV}$. These upper limits are two orders of magnitude below that estimated in thermal models, which have proven to quantitatively describe the yields of hadrons and produced nuclei including hypertriton, which has a binding energy of less than $150 \mathrm{keV}$.

In conclusion, none of the numerous dedicated experiments have found any evidence for the existence of the $H$ dibaryon. From the observation of double- $\Lambda$ hypernuclei a deeply bound $H$ dibaryon with a binding energy of more than $7 \mathrm{MeV}$ relative to the $\Lambda \Lambda$ threshold can be excluded. There have been many dedicated experiments to search for a $H$ dibaryon being either weakly bound or unbound. They provided stringent upper limits for its production in various processes, which make its existence very unlikely. However, as of yet none of them could completely rule out its existence.

\section{The New Era of Exclusive and Kinematically Complete High-Statistics Measurements at CELSIUS and COSY}

A conclusion drawn from the experiences made in the dibaryon rush era is that any meaningful dibaryon search should be undertaken with a dedicated experimental equipment, which is suited to provide exclusive and kinematically complete measurements of high statistics and high four-momentum resolution. In the nineties programs were set up at the storage rings CELSIUS and COSY, which allowed the search for dibaryon resonances with dedicated equipment within the official program for systematic studies of 
elastic scattering and meson production.

The COoler SYnchtron COSY at the research center Jülich has been providing high-brilliance polarized and unpolarized proton and deuteron beams. The beam cooling has been achieved by electron and stochastic cooling, respectively. For energy-dependence studies - like resonance and threshold issues it has been possible to ramp the beam with sub-MeV energy resolution from lowest to highest energies. That way the energy dependence of observables could be measured in a single run with a minimum of systematic errors. Whereas at CELSIUS the highest proton beam energy was $1.45 \mathrm{GeV}$, it was 2.88 $\mathrm{GeV}$ at COSY. For a review of the hadron physics program carried out at COSY from its start in 1993 until its end in 2014, see Ref. [294, 295].

\subsection{No signal for Narrow Resonances in the pp system}

The EDDA experiment at COSY provided ideal conditions for measuring the elastic proton-proton scattering over nearly the full angular range both with polarized beam and polarized target. In energy scan runs the energy range between $T_{p}=0.5-2.5 \mathrm{GeV}$, i.e. between $\pi$ and $\phi$ production thresholds, could be scanned with sub-MeV resolution in with high statistics [296, 297, 298, 299, 300, 301, 302, This provided the instrumental and analytical possibility to reveal even very narrow, weakly excited dibaryon resonances 1 in the mass range of $2200-2800 \mathrm{MeV}$.

With EDDA angular distributions over nearly the complete angular range have been obtained for the differential cross section [296, 297], the analyzing power [298, 299] as well as for the spin correlation observables $A_{N N}, A_{S S}$ and $A_{S L}$ [300, 301, 302]. As an example Fig. 5 shows the measured energy dependence of the differential cross section at center-of-mass polar angles $\Theta_{\text {c.m. }}=41^{\circ}, 55^{\circ}, 75^{\circ}$ and $89^{\circ}$ in comparison with previous measurements. It gets appearent that the EDDA measurements set a new standard in the experimental results for $N N$ scattering - both in quality and in quantity.

Similar to the examples shown in Fig. 5 all observables measured at EDDA do not show any statistical significant excursions, which could be a hint for narrow dibaryons. A quantitative search for dibaryons has been carried out for resonances in the energy range $\sqrt{s}=2.2-2.8 \mathrm{GeV}$ with total widths in the range $\Gamma=10-100 \mathrm{MeV}$ 302. Such putative resonances were added to various partial waves in phase-shift analyses of the data with the only free resonance parameter being the elastic partial width $\Gamma_{e l}$ expressed via the elasticity parameter $\eta_{e l}=\Gamma_{e l} / \Gamma$. The upper limits for $\eta_{e l}$ for exclusion with a confidence level of $99 \%$ are given in Ref. [302]. The obtained upper limits for $\eta_{e l}$ range between 0.03 0.10 allowing thus only isovector resonances, which hardly couple to the elastic channel. As an example Fig. 6 displays the fit to the data assuming a resonance in the ${ }^{1} S_{0}$ partial wave at $\sqrt{s}=2.7 \mathrm{GeV}$ with a width of $\Gamma=50 \mathrm{MeV}$ and an elasticity of $\eta_{e l}=0.043$, the value which is already excluded by the data with a confidence level of $99 \%$. Such a resonance was predicted in Ref. [86] and indications for it were claimed by the observation of some odd structure in the spin correlation parameter $A_{00 n n}$ [371]. EDDA provides no evidence for such a resonance.

Though no indications for any narrow resonances in the proton-proton system have been found, this EDDA data set is of indispensable value, since it constitutes the backbone of high-quality protonproton scattering data in the SAID data base [304] fixing the empirical partial-wave amplitudes with unprecedented precision. Unfortunately the EDDA experiment was not continued to examine also the proton-neutron scattering with similar precision - historically possibly a big mistake, as we will see in section 10 .

\subsection{No Narrow Resonances in Hyperon Production - except of $\Sigma N$ cusp}

At COSY the time-of-flight spectrometer TOF was constructed for the dedicated systematic study of hyperon production in $p p \rightarrow \Lambda p K^{+}, p p \rightarrow \Sigma^{0} p K^{+}$and $p p \rightarrow \Sigma^{+} p K^{0}$ reactions. The hermetic detector

\footnotetext{
${ }^{1}$ Occasionally the acronym EDDA has been interpreted also as "Elastic Dibaryons Dead or Alive" 303].
} 


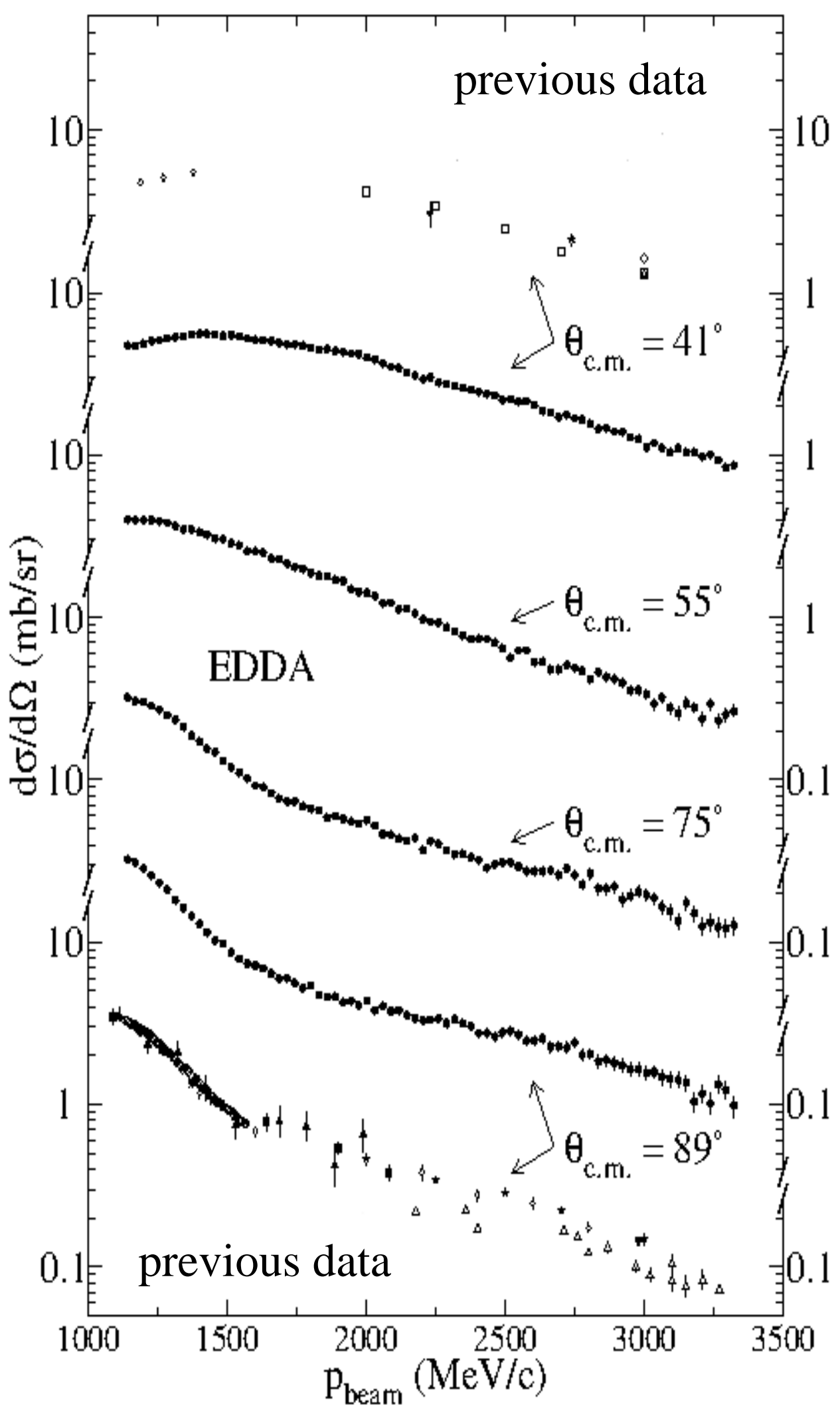

Figure 5: Energy dependence of the differential cross section for $p p$ elastic scattering in the range of beam momenta $p_{\text {beam }}=1.1-3.3 \mathrm{GeV} / \mathrm{c}$ corresponding to beam energies of $T_{\text {beam }} \approx 0.5-2.5 \mathrm{GeV}(\sqrt{s}$ $=2.1-2.8 \mathrm{GeV}$ ). Shown are results for $\Theta_{c . m .}=41^{\circ}, 55^{\circ}, 75^{\circ}$ and $89^{\circ}$ The EDDA data are shown by solid squares. For $\Theta_{\text {c.m. }}=41^{\circ}$ and $89^{\circ}$ they are compared to previous results. From [296]. 


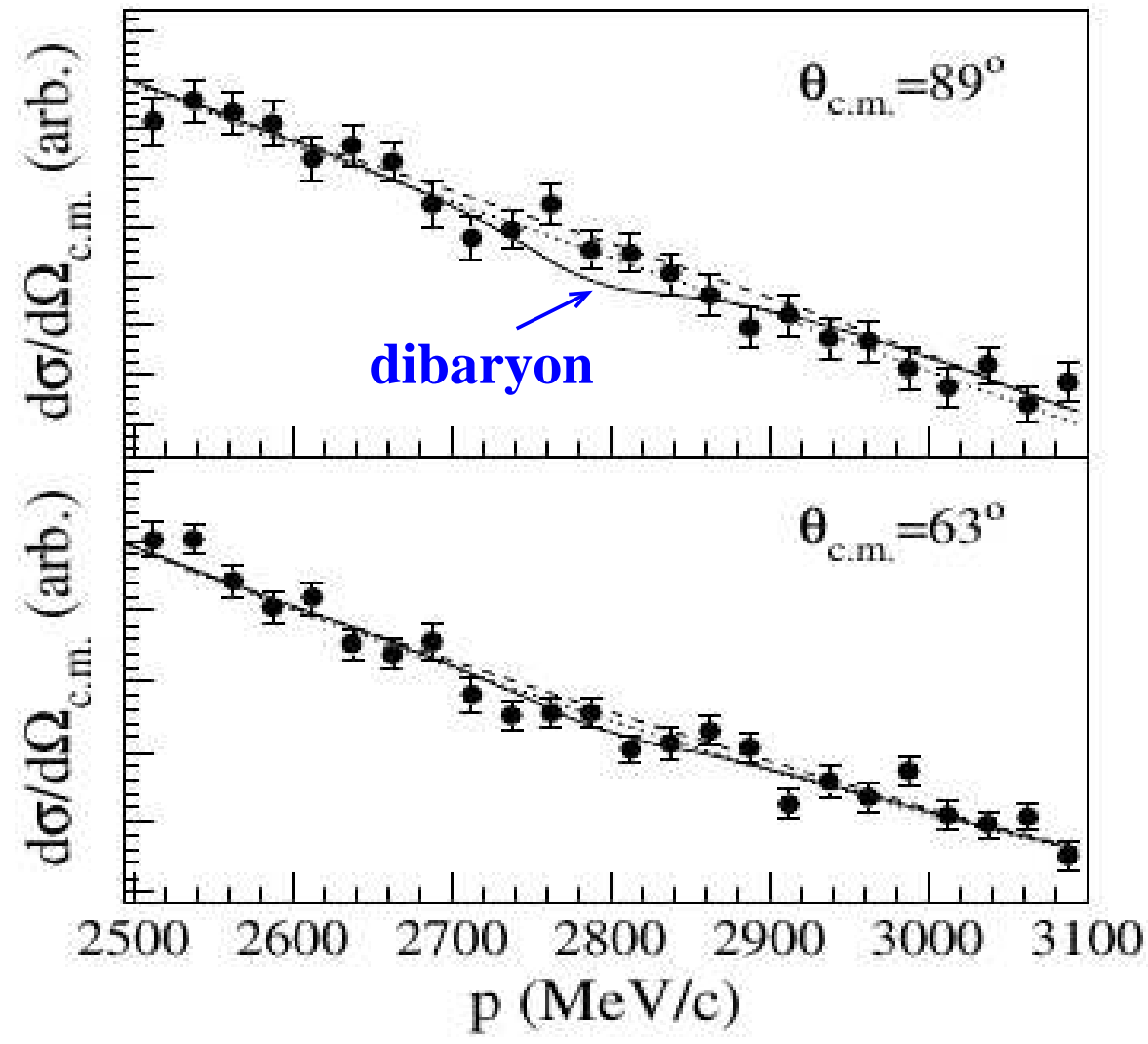

Figure 6: EDDA results for the differential cross section for $p p$ elastic scattering in the range of beam momenta $p_{\text {beam }}=2.5-3.1 \mathrm{GeV} / \mathrm{c}$ for the scattering angles $\Theta_{c . m} .=89^{\circ}$ and $63^{\circ}$ in comparison with results from phase-shift analyses with (solid lines) and without (dashed lines) including a putative dibaryon resonance in the ${ }^{1} S_{0}$ partial wave at $\sqrt{s}=2.7 \mathrm{GeV}$ with a total width of $\Gamma=50 \mathrm{MeV}$ and an elasticity $\eta_{e l}=0.043$. By the $\chi^{2}$ fit criterion such a resonance is excluded with a confidence level of $99 \%$. The dotted line shows the nonresonant background described by the phase-shift analysis in presence of such a resonance. From [302]. 
has been able to cover practically the full reaction phase space. Highly segmented start and stop scintillation detectors allowed time-of-flight measurements of charged ejectiles and vertex reconstruction. Fiber and straw-tube detectors in-between enabled in addition the vertex reconstrcution of decaying hyperons. That way exclusive and kinematically complete measurements have been possible over the full phase space. Since most of these measurements were not only kinematically complete, but even overdetermined, kinematic fits with overconstraints could be carried out with the consequence of highly improved energy resolutions in invariant-mass spectra in the order of few $\mathrm{MeV}$ and better.

The $p p \rightarrow \Sigma^{+} p K^{0}$ reaction has been investigated at three beam energies $T_{p}=2.16,2.26$ and 2.40 $\mathrm{GeV}$ [66]. It is well described in all its differential observables by $N^{*}$ excitation via $t$-channel meson exchange and its subsequent decay $N^{*} \rightarrow \Sigma^{+} K^{0}$. This is particularly true for the $\Sigma^{+} p$ invariant-mass spectrum, which spans the mass range from threshold up to $2290 \mathrm{MeV}$ and which gives no indication for narrow dibaryon structures of statistical significance.

The $p p \rightarrow \Lambda p K^{+}$reaction has been measured at numerous beam energies in the range $T_{p}=1.92$ $-2.49 \mathrm{GeV}$ [305, 306, 67, 307, 308], partly even with polarized beam [309, 60]. Of particular interest here are the high-resolution and high-statistics measurements, which cover the $\Lambda p$ invariant-mass range from threshold up to $2.3 \mathrm{GeV}$ [307, 308, 309].

Fig. 7] shows the $\Lambda p$ invariant-mass spectra obtained with COSY-TOF at $T_{p}=2.28 \mathrm{GEV}$ with a mass resolution of $\sigma_{m}=2.6 \mathrm{MeV}$ and at $T_{p}=2.16 \mathrm{GeV}$ with $\sigma_{m}=1.1 \mathrm{MeV}$. The only obvious structures are the enhancement at the $\Lambda p$ threshold due to the $\Lambda p$ final-state interaction and the narrow spike at the thresholds of $\Sigma^{+} n$ and $\Sigma^{0} p$ at $2129 \mathrm{MeV}$ and $2131 \mathrm{MeV}$, respectively. A coupled-channel treatment of the cusp due to these thresholds gives a good account for the low-energy side of the observed structure. However, for the high-energy side the calculation falls off a bit too fast.

This situation is very similar to that observed in $K^{-}$absorption on the deuteron - as discussed already in detail in section 4.2.5. In particular, the high-resolution measurement of Tan [163] displays also such a high-energy shoulder. The high-resolution data shown in Fig. 7, middle, indicate some narrow fluctuation below $3 \sigma$ confidence at $2.146 \mathrm{GeV}$ on this high-energy slope. A very recent highstatistics and high-resolution measurement [308] shows again such a fluctuation, but now at a slightly smaller mass. From this we conclude that at present there is no statistical solid evidence for a narrow structure beyond the $\Sigma N$ cusp. However, there is solid evidence for a surplus of cross section at the high-energy side of the cusp, which so far is not understood theoretically.

Also, the peak at the $\Sigma N$ threshold is observed to vanish in the $p p \rightarrow \Lambda p K^{+}$reaction towards smaller incident energies. At $T_{p}=1.92 \mathrm{GeV}$ it is already invisible in the data [60]. There the cross section ratio between $\Lambda$ and $\Sigma^{0}$ production is five times larger than at $T_{p}=2.28 \mathrm{GeV}$ due to the $\Lambda p$ FSI. This is in accord with the expection for a cusp effect, where the channel-coupling depends on this cross section ratio.

In Ref. 308, it is shown that the shallow fall-off at the high-energy side of the cusp can be accounted for phenomenologically in the framework of a Flatté ansatz, if a (broad) virtual state in the $\Lambda p$ system in the mass range $2200-2231 \mathrm{MeV}$ is assumed. Clearly, more profound theoretical investigations are needed.

The $p p \rightarrow \Lambda p K^{+}$reaction was also measured exclusively and kinematically complete with the HADES detector at GSI at a beam energy of $3.5 \mathrm{GeV}$ [310]. Though the solid angle coverage is substantially less than $4 \pi$, a reliable correction based on partial-wave analysis could be achieved. As a result 275] no statistically significant structures for narrow $\Lambda p$ dibaryon resonances in the mass range below $2600 \mathrm{MeV}$ have been revealed - see Fig. 6 of Ref. [275].

Summarizing, aside from the $\Lambda N$ FSI and the possibly not yet completely understood $\Sigma N$ cusp effect there are no statistically significant structures giving indications for resonances in $\Lambda N$ and $\Sigma N$ systems with masses below $2600 \mathrm{MeV}$. 

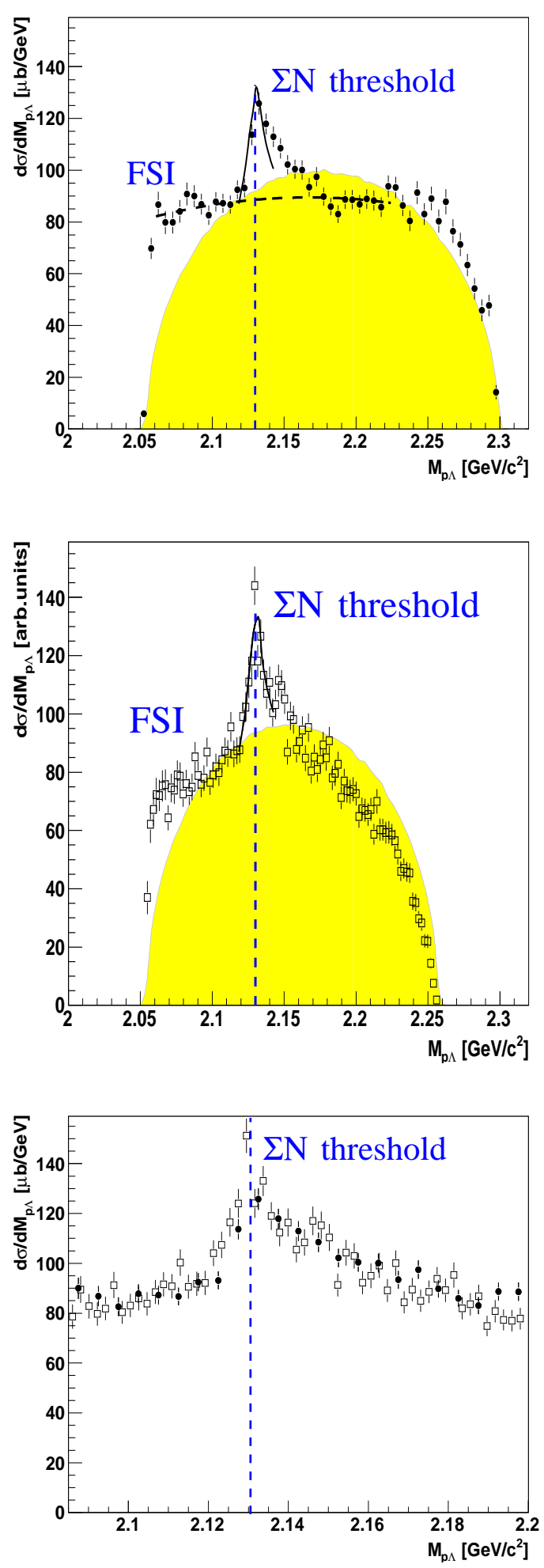

Figure 7: Distributions of the $\Lambda p$ invariant mass as obtained from COSY-TOF measurements at $T_{p}=$ $2.28 \mathrm{GeV}$ with an invariant-mass resolution of $\sigma_{m}=2.6 \mathrm{MeV}$ (top) and at $T_{p}=2.16 \mathrm{GeV}$ with $\sigma_{m}=$ 1.1 MeV (middle). The shaded (yellow) area shows the distribution expected from pure phase space. The solid curve gives the shape of the $\Sigma N$ cusp distribution as obtained from a coupled-channel treatment of this phenomenon - averaged over the experimental resolution and fitted in height to the data at the low-energy side of the cusp. The cusp is assumed to sit upon a smooth background represented by the dashed line. At the bottom both data sets are compared in the region of the cusp. Aside rom a possible slight shift of about $1 \mathrm{Mev}$, which is within the uncertainty of the energy calibrations both data sets coincide, if the different energy resolutions are taken into account. From [307]. 


\subsection{No signal from the anticipated $N N \pi$ Resonance $d^{\prime}(2065)$}

The WASA experiment, until 2005 installed at CELSIUS and thereafter at COSY, provided the ideal requirements for measuring multi-pion production in unprecedented quality. Though the $\pi N$ system was known to provide a wealth of baryon resonances and also first meaningful theoretical calculations predicted the $\Delta \Delta$ system to play a dominant role in the two-pion production process, the data base was still scarce at that time. A reason was that afore no detector was available on a nucleon-nucleon machine, which was able to cover the full phase space of complex reactions combined with high statistics.

Since $p p$ collisions are experimentally much easier to conduct than $p n$ collisions, the WASA collaboration started to systematically measure the $p p \rightarrow p p \pi^{+} \pi^{-}$reaction close to threshold.

One of the first aims was the search for the anticipated $N N \pi$ resonance $d^{\prime}(2065)$, which was introduced for the explanation of the resonance-like forward-angle cross section observed in the pionic double-charge exchange reaction on nuclei at incident pion energies around $50 \mathrm{MeV}$ - see section 5 .

And, indeed, already the first test run for measuring two-pion production close to threshold revealed a narrow, $4 \mathrm{MeV}$ broad structure in the $p p \pi^{-}$invariant mass spectrum at $2.063 \mathrm{GeV}$, i.e. right at the position, where $d^{\prime}(2065)$ was expected [311]. However, a follow-up high-statistics run revealed this structure to be partly a statistical fluctuation and partly an instrumental artifact [312]. No statistical significant $(>3 \sigma)$ narrow structures have been observed, neither in the $p p \pi^{-}$nor in the $p p \pi^{+}$spectrum, see Fig. 8 .

An upper limit of $\sigma<20 \mathrm{nb}$ has been derived for the production of any narrow $N N \pi$ dibaryons in the mass range from 2020 - $2085 \mathrm{MeV}$. This includes both isoscalar and isotensor dibaryons. This upper limit is more than an order of magnitude smaller than estimated [239, 313] for the $d^{\prime}(2065)$ production in this reaction. Also a measurement of the $p p \rightarrow p p \pi^{+} \pi^{-}$reaction at $T_{p}=0.793 \mathrm{GeV}$ at COSY-TOF, which covers $p p \pi$ invariant masses up to $2095 \mathrm{MeV}$, finds no evidence for a narrow dibaryon strcuture [314, 315].

In conclusion Ref. 312] states that

- either $d^{\prime}$ does not exist at all, or

- its production cross section in $p p$ collisions is smaller than expected from theoretical estimates [239, 313], or

- the mass of the free $d^{\prime}$ is outside the range investigated here, or

- it exists only in the nuclear medium.

Effects of the nuclear medium on the effective mass of dibaryons with particular emphasis on $d^{\prime}$ have been investigated in Ref. [316]. There a decreasing dibaryon mass with increasing nuclear density is predicted. The data on the pionic double-charge exchange reaction, indeed, exhibit some tendency of a decreasing peak energy, i.e. of a decreasing $d^{\prime}$ mass with an increasing mass number, i.e. increasing density of the nucleus [227]. According to Ref. [316] the vacuum dibaryon mass is expected to be about $15 \%$ larger than that at nuclear density $\rho_{0}$, i.e. in the interior of ${ }^{208} \mathrm{~Pb}$. Since the pionic doublecharge exchange reaction occurs predominantly at the nuclear surface, i.e. at roughly $\rho_{0} / 2$, this would mean that the vacuum mass of $d^{\prime}$ is expected to be near $2200 \mathrm{MeV}$, which is already far above the $N N \pi$ threshold. In consequence $d^{\prime}$ could decay easily into the $N N \pi$ system and hence would no longer appear as a narrow resonance.

\subsection{No dibaryon signal in pp-induced two-pion production}

Before the start of the two-pion production programs at CELSIUS and COSY there was only very little experimental information on this process. Most of the previous results originated from low-statistics 

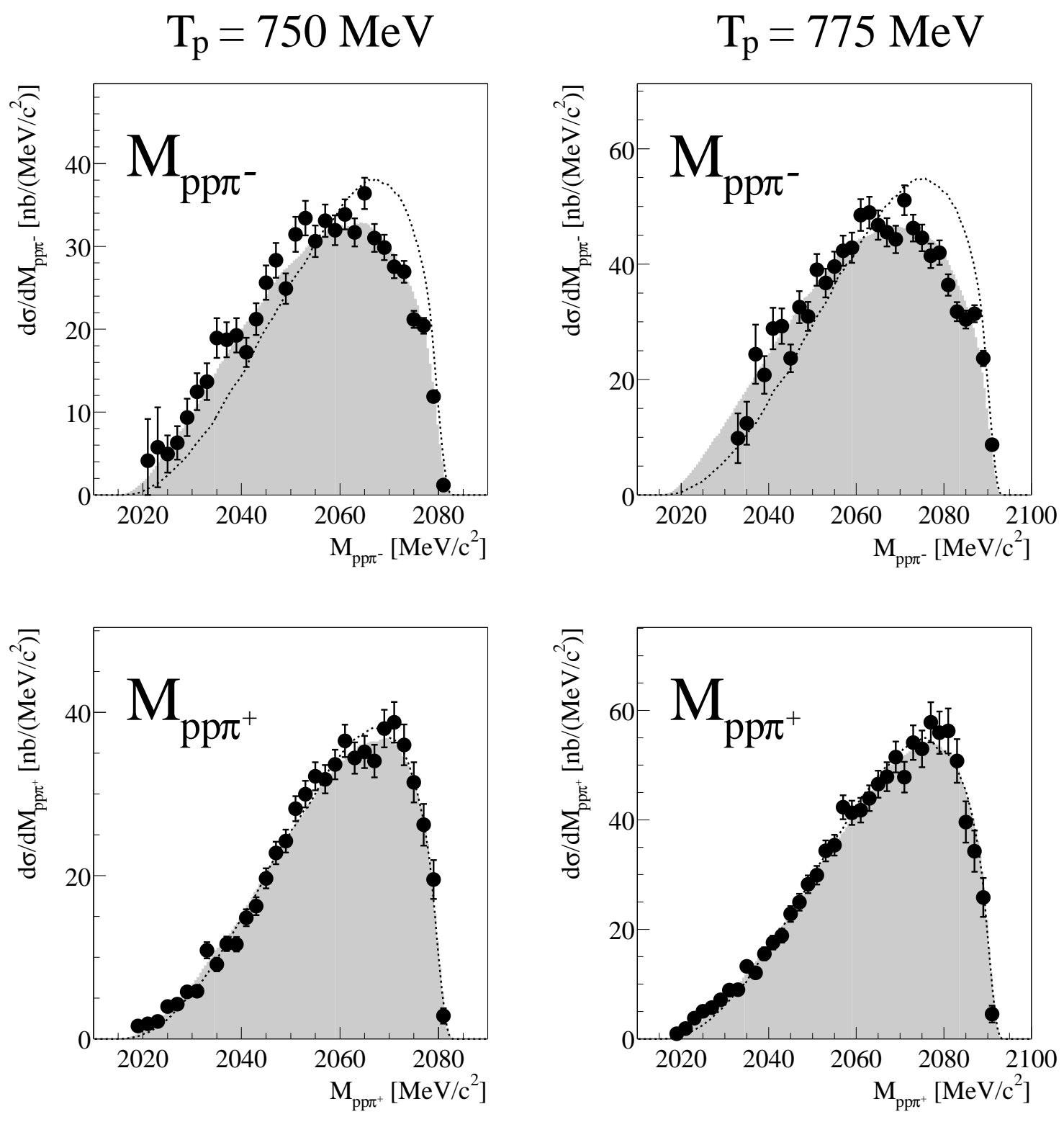

Figure 8: Distributions of the $p p \pi^{-}$and $p p \pi^{+}$invariant masses $M_{p p \pi^{-}}$(top) and $M_{p p \pi^{+}}$(bottom), respectively, obtained from measurements of the $p p \rightarrow \pi^{+} \pi^{-}$reaction at $T_{p}=0.75 \mathrm{GeV}$ (left) and 0.775 $\mathrm{GeV}$ with the WASA/PROMICE detector at CELSIUS. The solid circles represent the experimental results. The dotted lines indicate a pure phase-space distribution and the shaded areas give a model calculation for the excitation of the Roper resonance and its decay into the two-pion channel. From Ref. [312]. 
bubble-chamber measuerements [317, 318, 319, 320, 321, 322]. Fig. 9] shows their results for the total cross sections by the open symbols. Due to the very limited statistics differential distributions were even more scarce and only available for the $p p \pi^{+} \pi^{-}$channels at energies far above threshold.

In this situation the two-pion production program started at CELSIUS with exclusive kinematically complete high-statistics measurements with particular emphasis on the threshold region, the most suitable place for revealing narrow dibaryon resonances. Starting first with the WASA/PROMICE detector setup, followed by the completed WASA detector at CELSIUS and finally at COSY all $p p$ induced, i.e. isovector two-pion production channels from threshold up to $\sqrt{s}=2.5 \mathrm{GeV}$ have been studied [323, 324, 325, 326, 327, 328, 329, 330, 331 complemented by a corresponding program with polarized beam at COSY-TOF [314, 332]. The results for the total cross sections are shown in Fig. 9 by solid symbols. Special emphasis has been put on the $p p \pi^{0} \pi^{0}$ channel, where the bubble-chamber results suggested a kink in the total cross section at $T_{p}=1.2 \mathrm{GeV}$. The WASA results confirmed this kink in high-statistics measurements. In subsequent isospin decomposition of the total cross sections according to the formalism presented in Refs. [317, 333] it could be demonstrated [327] that excitation of the Roper resonance by $t$-channel meson exchange and its subsequent decay into the $N \pi \pi$ channel as well as the mutual excitation of the colliding nucleons into their first excited state, the $\Delta$ resonance with subsequent $N \pi$ decay ( $t$-channel $\Delta \Delta$ excitation), are the dominant processes - as properly predicted by the Valencia theory group [334] and later-on also by IHEP calculations [335]. The $\Delta \Delta$ excitation by conventional $t$-channel meson exchange is depicted schematically in Fig. 10, top.

In this isospin decomposition the total cross sections are decomposed into contributions from reduced matrix elements $M_{I_{N N}^{f} I_{\pi \pi} I_{N N}^{i}}$, where $I_{N N}^{i}$ and $I_{N N}^{f}$ denote the isospin of the nucleon pair in initial and final state, respectively, and where $I_{\pi \pi}$ is the isospin of the produced pion pair. For a specific process these matrix elements depend on the isospin coupling coefficients. For the $\Delta \Delta$ process, e.g. the matrix elements are proportional to the respective $9 \mathrm{j}$-symbol for isospin recoupling:

$$
M_{I_{N N}^{f} I_{\pi \pi} I_{N N}^{i}}^{\Delta \Delta} \sim \hat{I}_{\Delta_{1}} \hat{I}_{\Delta_{2}} \hat{I}_{N N}^{f} \hat{I}_{\pi \pi}\left\{\begin{array}{ccc}
I_{N_{1}} & I_{\pi_{1}} & I_{\Delta_{1}} \\
I_{N_{2}} & I_{\pi_{2}} & I_{\Delta_{2}} \\
I_{N N}^{f} & I_{\pi \pi} & I_{\Delta \Delta}
\end{array}\right\},
$$

where $N_{i}$ and $\pi_{i}$ couple to $\Delta_{i}$ for $i=1,2$ and $\hat{I}_{\alpha}=\sqrt{2 I_{\alpha}+1}$ and $I_{\Delta \Delta}=I_{N N}^{i}-$ as outlined in Refs. [327, 336].

The resulting partial cross sections $\sigma_{101}, \sigma_{011}, \sigma_{111}$ and $\sigma_{121}$ are shown in Fig. 9 by the drawn lines and shaded areas, respectively. The isospin decomposition suggests that also a higher-lying $\Delta$ excitation, preferably the $\Delta(1600)$ could play some role, in particular in the $n n \pi^{+} \pi^{+}$channel. The isospin decomposition reveals the kink in the total cross section of the $p p \pi^{0} \pi^{0}$ channel as being due to destructive interference effects between isoscalar and isotensor $\pi \pi$ contributions as well as between $N^{*}$ and $\Delta \Delta$ contributions. I.e., in this isospin decomposition concept the kink does not appear to be a signature of the contribution of two dibaryon resonances $\left(\Delta N\right.$ configurations in ${ }^{3} F_{3}$ and ${ }^{1} G_{4} p p$ partial waves), as assumed recently [206]. However, it will be very interesting to see, whether the alternative concept 206] of isovector dibaryon resonance contributions in combination with low cut-off parameters as discussed at the end of section 4.4 for single-pion production will lead also here to a quantitative description of data on all isovector two-pion production channels.

Still much more information about the reaction process is, of course, contained in the various differential distributions, which all are available in kinematically complete measurements. By fine adjustments of the resonance parameters in the Valencia model calculations all differential distributions could be quantitatively described by the conventional process of $t$-channel meson exchange leading to the excitation of the Roper resonance close to threshold followed at higher energies by the excitation of the $\Delta \Delta$ system. It has been demonstrated that the Roper excitation and its decay via the interfering routes $N^{*} \rightarrow N \sigma \rightarrow N \pi \pi$ and $N^{*} \rightarrow \Delta \pi \rightarrow N \pi \pi$ lead to very characteristic patterns in the differential 
distributions, especially in the $\pi \pi$ invariant-mass distribution [314, 323, 325, 326]. In the $\Delta \Delta$ region it is in addition the $p \pi$ invariant mass distributions, which exhibit a characteristic pattern [329].

As a result of these systematic studies it was found that isovector induced two-pion production can be quantitatively well understood by the conventional process of $t$-channel meson exchange leading to the excitation of the Roper resonance close to threshold followed by the excitation of the $\Delta \Delta$ system at higher energies. To some extent also the $\Delta(1600)$ excitation is seen to play some role. But no hint for an exotic resonance production is observed.

\section{Towards the First Extraordinary Dibaryon}

The situation changed drastically, when $p n$-induced two-pion production was looked at, though the pathway towards a dibaryon resonance was still far from being straightforward.

\subsection{Following a trace: the $A B C$ effect}

Since the sixties there existed an unsolved problem concerning the two-pion production in cases, when the participating nucleons merge into a nuclear boundstate - the so-called double-pionic fusion reactions.

In 1960 Abashian, Booth and Crowe [337] noticed in the inclusively measured $p d \rightarrow{ }^{3} \mathrm{HeX}$ reaction that there is a substantial enhancement in the ${ }^{3} \mathrm{He}$ missing mass spectrum corresponding to the emission of two pions. This enhancement cumulated at masses close to the threshold of twice the pion mass. Subsequent measurements demonstrated that this effect - corresponding to a low-mass enhancement in the spectrum of the $\pi \pi$ invariant mass - occurs in the double-pionic fusion reactions $p n \rightarrow d \pi \pi$, $p d \rightarrow{ }^{3} \mathrm{He} \pi \pi$ and $d d \rightarrow{ }^{4} \mathrm{He} \pi \pi$, but not in the fusion to ${ }^{3} \mathrm{H}$ in the reaction $p d \rightarrow{ }^{3} \mathrm{H} \pi \pi[338,339$, 340, 341, 342, 343, 344, 345, 346, 347, 348. Since in the latter case the pion pair must be in an isovector state, it was concluded that the observed enhancement must be correlated with the creation of an isoscalar pion pair.

First attempts for explaining this effect by an unusually strong $\pi \pi$ interaction or a narrow $\sigma$ meson had soon to be abandoned due to conflicts with experimental results from other two-pion production processes.

Since this low-mass enhancement was especially pronounced at beam energies, where the colliding nucleons could undergo a mutual excitation into their first excited state, the $\Delta(1232)$, it was finally proposed [349] that the conventional $\Delta \Delta$ excitation by $t$-channel meson exchange - as depicted schematically in Fig. 10, top - might be the reason for the observed low-mass enhancement. However, according to this explanation there should also be a high-mass enhancement in the $\pi \pi$ invariant-mass spectrum. And indeed, a number of inclusive measurements exhibited such a putative enhancement. But as pointed out in Ref. [346] this high-mass region region corresponded also to the kinematical region of $\eta$ and three-pion production, which could not be separated in the inclusive measurements.

So, since finally no conclusive explanation could be found for the observed low-mass enhancement it was named in the literature "ABC" effect after the initials of the authors Abashian, Booth and Crowe, who noticed this enhancement first.

Since previous measurements either were low-statistics bubble-chamber or inclusive single-arm measurements conducted at a few scattering angles, the CELSIUS/WASA collaboration decided to reexamine the ABC effect - with the WASA detector setup still at CELSIUS - and to perform exclusive and kinematically complete measurements of the double-pionic fusion reactions.

In a first step the reactions $p d \rightarrow{ }^{3} \mathrm{He} \pi^{0} \pi^{0}$ and $p d \rightarrow{ }^{3} \mathrm{He} \pi^{+} \pi^{-}$were looked at, for which data already existed from runs dedicated to the $\eta$ production at threshold. As a result it was found [350] that there is, indeed, a highly visible low-mass enhancement but no high-mass enhancement as seen in previous 

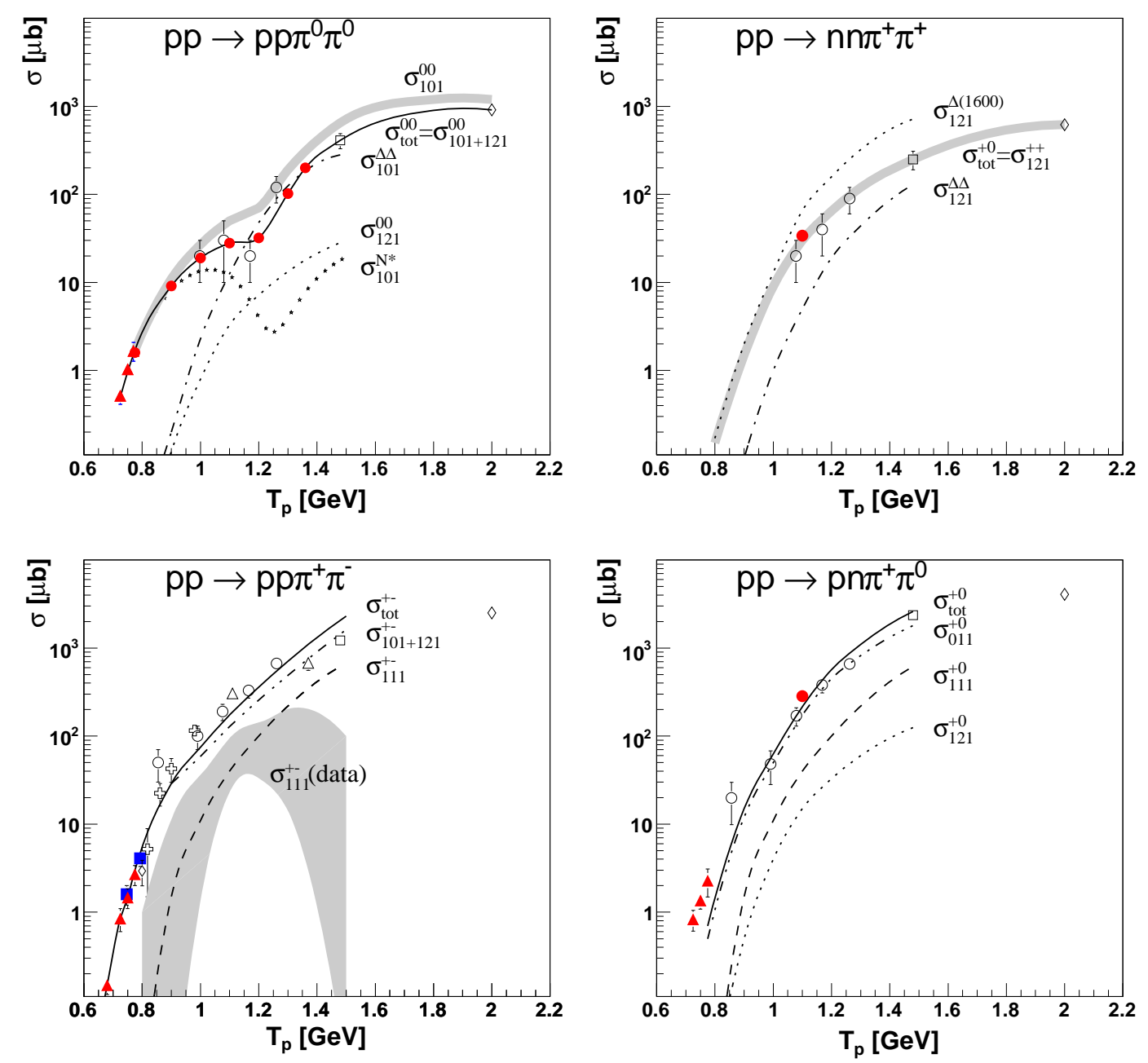

Figure 9: Energy dependence of the $p p$-induced total cross sections for two-pion production and their isospin decomposition. Open symbols denote results from bubble-chamber measurements [317, 318, 319, 320, 321, 322], solid symbols represent measurements from WASA/PROMICE (triangles) [323, 324, 325], CELSIUS/WASA (circles) [326, 327, 328, 329, 330] and COSY-TOF (squares) [314]. At the top the reaction channels $p p \rightarrow p p \pi^{0} \pi^{0}$ (left) and $p p \rightarrow n n \pi^{+} \pi^{+}$(right) are shown together with their isospin decomposition. the drawn lines show the extracted contributions $\sigma_{121}, \sigma_{101}$ and $\sigma_{101+121}$ as indicated in the figures. Also shown are the decomposition of $\sigma_{121}$ into contributions from $\Delta \Delta$ and $\Delta(1600)$ and of $\sigma_{101}$ into $N^{*}$ and $\Delta \Delta$ contributions. At the bottom the reaction channels $p p \rightarrow p p \pi^{+} \pi^{-}$(left) and $p p \rightarrow p n \pi^{+} \pi^{0}$ (right) are shown. The broken lines show the extracted contributions of $\sigma_{121}, \sigma_{101}$ and $\sigma_{101+121}$. The large shaded area indicates $\sigma_{111}$ as derived by direct comparison of the data for the $p p \rightarrow p p \pi^{+} \pi^{-}$reaction with $\sigma_{101+121}$. From Ref. [327. 


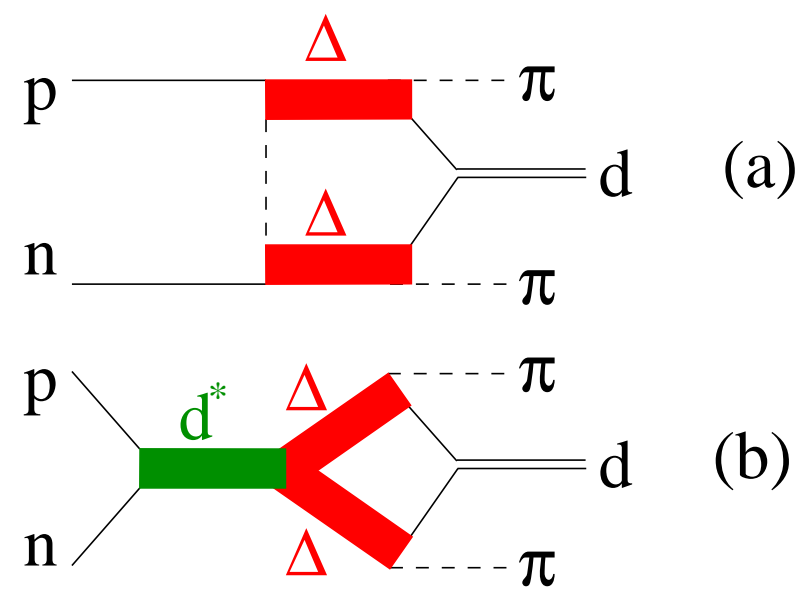

Figure 10: Schematic representation of the process $p n \rightarrow d \pi \pi$ by $\Delta \Delta$ excitation via $t$-channel meson exchange (a) or by $s$-channel formation of the $d *(2380)$ resonance with subsequent decay via an intermediate $\Delta \Delta$ system (b). From [362].

inclusive measurements. As already suspected in Ref. [346] this putative high-mass enhancement was in reality due to $\eta$ and three-pion production.

Having now an established strong low-mass enhancement, but the $t$-channel $\Delta \Delta$ concept no longer as a valid explanation, it was suggested that the ABC effect might be a sign of a quasi-bound $\Delta \Delta$ system [350]. However, if this denotes a resonance phenomenon in the system of two baryons, then it would need to show up also as a resonance structure in the total cross section of the basic double-pionic fusion, which is the one to the deuteron.

\subsection{Exclusive and Kinematically Complete Measurement of the Basic Double-Pionic Fusion Process}

For this endeavor the experimental technique had to be changed, since free neutrons are not available - neither as beam or as target particles. Hence the quasi-free process with deuterons being the source for quasi-free neutrons has been utilized by the WASA collaboration - a method established before in many other experiments, see e.g. Refs. [351, 352]. This process has the additional advantage that the Fermi motion of the neutron within the deuteron provides a range of collision energies with a single beam energy setting. That way the energy dependence of a reaction can be conveniently scanned, which is particularly well suited for the search for narrow resonances. However, a precondition for a successful use of the quasi-free process is that the four-momenta of all ejectiles are determined experimentally, which necessitates exclusive and kinematically complete measurements.

First such measurements were conducted with WASA still at CELSIUS. Though the statistics was quite limited there due to low beam current, a clear correlation of the ABC effect with a peak-like structure in the total cross section (open triangles in Fig. 12, middle panel) could be revealed in the $p n \rightarrow d \pi^{0} \pi^{0}$ reaction, which was measured via the quasi-free process $p d \rightarrow d \pi^{0} \pi^{0}+p_{\text {spectator }}$ [27]. As it turned out later, this was the golden channel for the dibaryon issue, since it possesses a very low background from conventional processes. Experimentally it was only accessible with instruments like WASA being able to detect both charged and uncharged particles over essentially the full solid angle. Hence it is not of a surprise that there are no data for this channel from previous experiments.

Follow-up measurements of this reaction - meanwhile with WASA at COSY providing two orders of magnitude higher statistics - revealed a pronounced Lorentzian structure in the total cross section corresponding to a resonance at $\sqrt{s}=2.37 \mathrm{GeV}$ with a width of only $70 \mathrm{MeV}$ [26], see Fig. 11. The resonance structure is about $90 \mathrm{MeV}$ below the mass of two $\Delta$ states and its width is more than three 
times narrower than that of a conventional $t$-channel $\Delta \Delta$ excitation.

Fig. 11. shows the measurement of the total cross section as well as a selection of differential distributions at $\sqrt{s}=2.38 \mathrm{GeV}$, the peak energy region. The shown data for the deuteron angular distribution (Fig. 11, on the left of the middle panel) are the result of two runs, one run with the spectator proton in the target, i.e., with the reaction $p d \rightarrow d \pi^{0} \pi^{0}+p_{\text {spectator }}$ (open circles), and another run with the spectator proton in the beam (reversed kinematics), i.e., with the reaction $d p \rightarrow p_{\text {spectator }}+d \pi^{0} \pi^{0}$ ( solid circles). That way the acceptance over the full angular range could be optimized. The data are in accordance with the Barshay-Temmer theorem [353], according to which the angular distribution of a purely isoscalar reaction has to be symmetric about $90^{\circ}$ in the center-of-mass system. The dashed line shows a fit with an expansion into Legendre Polynomials of order $=0,2,4$ and 6 corresponding to a total spin of the resonance of $J=3$. Together with the fact that the $p n \rightarrow d \pi^{0} \pi^{0}$ reaction is purely isoscalar, we get the quantum numbers $I\left(J^{P}\right)=0\left(3^{+}\right)$for this resonance structure. Due to its isoscalar character it is formally compatible with an excitation of the deuteron, hence it has been named $d^{*}$.

In further WASA measurements it has been demonstrated that below and above this resonance structure the angular distributions get flatter [355]. The fact that the angular distribution flattens out towards lower energies is not unexpected, since towards the reaction threshold we expect contributions only from lowest partial waves. However, the observation that the angular distribution gets again flatter at higher energies is not trivial. It is in support of the fact that the high spin of $J=3$ of the resonance requires an unusually large anisotropy of the angular distribution, which is larger than that of the conventional $t$-channel $\Delta \Delta$ process, which dominates at higher energies.

The Dalitz plot of this reaction is displayed in Fig. 11, on the right of the middle panel. It exhibits two distinctive features. First, there is a clear horizontal band, which is in accord with a $\Delta \Delta$ excitation in the intermediate state as it is also corroborated by the Dalitz plot projection on the vertical axis. This projection gives the distribution of the square of $d \pi^{0}$ invariant mass displayed on the left of the bottom panel. The second exceptional feature is the huge enhancement at low $\pi^{0} \pi^{0}$ invariant masses as borne out in the Dalitz projection on the horizontal axis. This provides the distribution of the square of the $\pi^{0} \pi^{0}$ invariant masses as displayed on the right of the bottom panel. This enhancement is the so-called ABC effect mentioned above. And as the WASA measurements demonstrate it is strictly correlated with the appearance of the $d^{*}$ resonance structure in the total cross section. It may be described phenomenologically by a vertex function in the decay of this resonance into the intermediate $\Delta \Delta$ state [26]. For alternative descriptions see Refs. [361, 362].

The purely isoscalar character of the observed resonance structure has been confirmed by the subsequent measurement of all three fusion reactions $p n \rightarrow d \pi^{0} \pi^{0}, p n \rightarrow d \pi^{+} \pi^{-}$and $p p \rightarrow d \pi^{+} \pi^{0}$ simultaneously and their isospin decomposition [28] - see Fig. 12. Whereas the first reaction is purely isoscalar, the third one is purely isovector. The second reaction is isospin mixed containing both isoscalar and isovector components. Its isospin decomposition yields [333]

$$
\sigma\left(p n \rightarrow d \pi^{+} \pi^{-}\right)=2 \sigma\left(p n \rightarrow d \pi^{0} \pi^{0}\right)+\frac{1}{2} \sigma\left(p p \rightarrow d \pi^{+} \pi^{0}\right)
$$

Fig. 12 shows the total cross sections of these three reactions multiplied by factors according to eq. (4). For the $p n \rightarrow d \pi^{+} \pi^{-}$and $p p \rightarrow d \pi^{+} \pi^{0}$ reactions there are also some results from previous measurements [358, 359, 317, 319, 328, 333], they are depicted by open symbols in Fig. 12, top and bottom. As mentioned already above there have been no previous measurements of the $p n \rightarrow d \pi^{0} \pi^{0}$ reaction, where the background from the conventional $t$-channel processes is smallest.

As Fig. 12 impressively demonstrates, there is no sign of the narrow resonance structure around 2.38 $\mathrm{GeV}$ to see in the data for the purely isovector $p p \rightarrow d \pi^{+} \pi^{0}$ reaction. The broad structure seen in this reaction with a width of about $2 \Gamma_{\Delta}$ and peaking around twice the $\Delta$ mass can be well described by the conventional $t$-channel $\Delta \Delta$ process [328] - see dashed line in the bottom panel of Fig. 12 ,

By use of isospin decomposition and recoupling of this $\Delta \Delta$ process - as described, e.g., in Ref. 

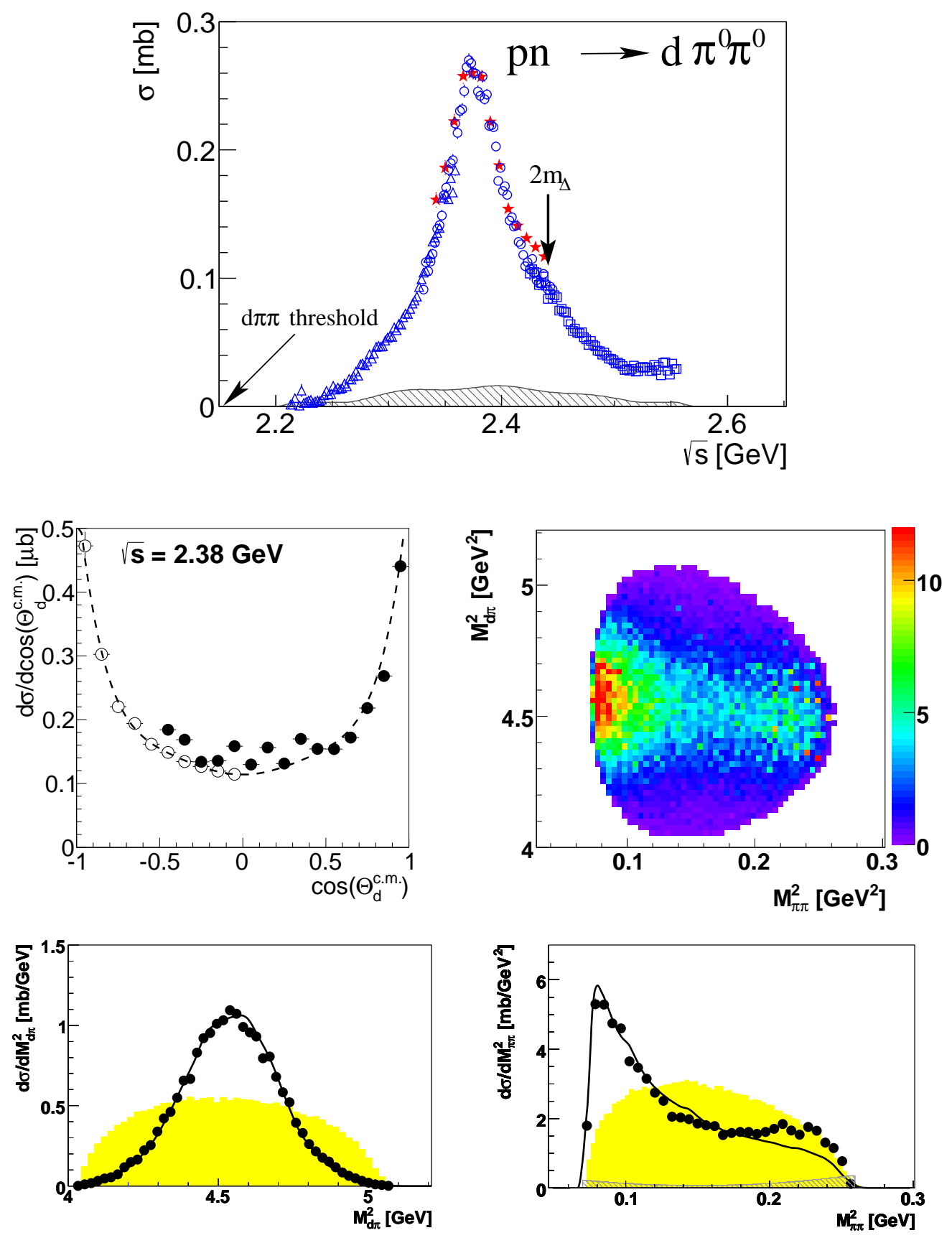

Figure 11: Measurements of the golden reaction channel $p n \rightarrow d \pi^{0} \pi^{0}$ with WASA at COSY. Top: total cross section exhibiting the pronounced resonance effect of $d^{*}(2380)$. The blue open symbols show the data of Ref. [26] properly normalized in absolute scale to the data of Ref. [28] plotted by red stars. The black shaded area gives an estimate of systematic uncertainties. Middle: deuteron angular distribution (left) and Dalitz plot (right) at the peak energy of $\sqrt{s}=2.38 \mathrm{GeV}$. Open and solid dots refer to measurements with the spectator proton in the target and in the beam (reversed kinematics), respectively. The dashed curve gives a Legendre fit with $L_{\max }=6$ corresponding to $\mathrm{J}=3$. Bottom: Projections of the Dalitz plot yielding the distributions of the squares of the $d \pi^{0}$ (left) and $\pi^{0} \pi^{0}$ (right) invariant masses. The large low-mass enhancement in the latter denote the so-called ABC effect. The solid lines represent a theoretical description of the process $p n \rightarrow d^{*}(2380) \rightarrow \Delta^{+} \Delta^{0} \rightarrow d \pi^{0} \pi^{0}$. From [26, 354, 355]. 

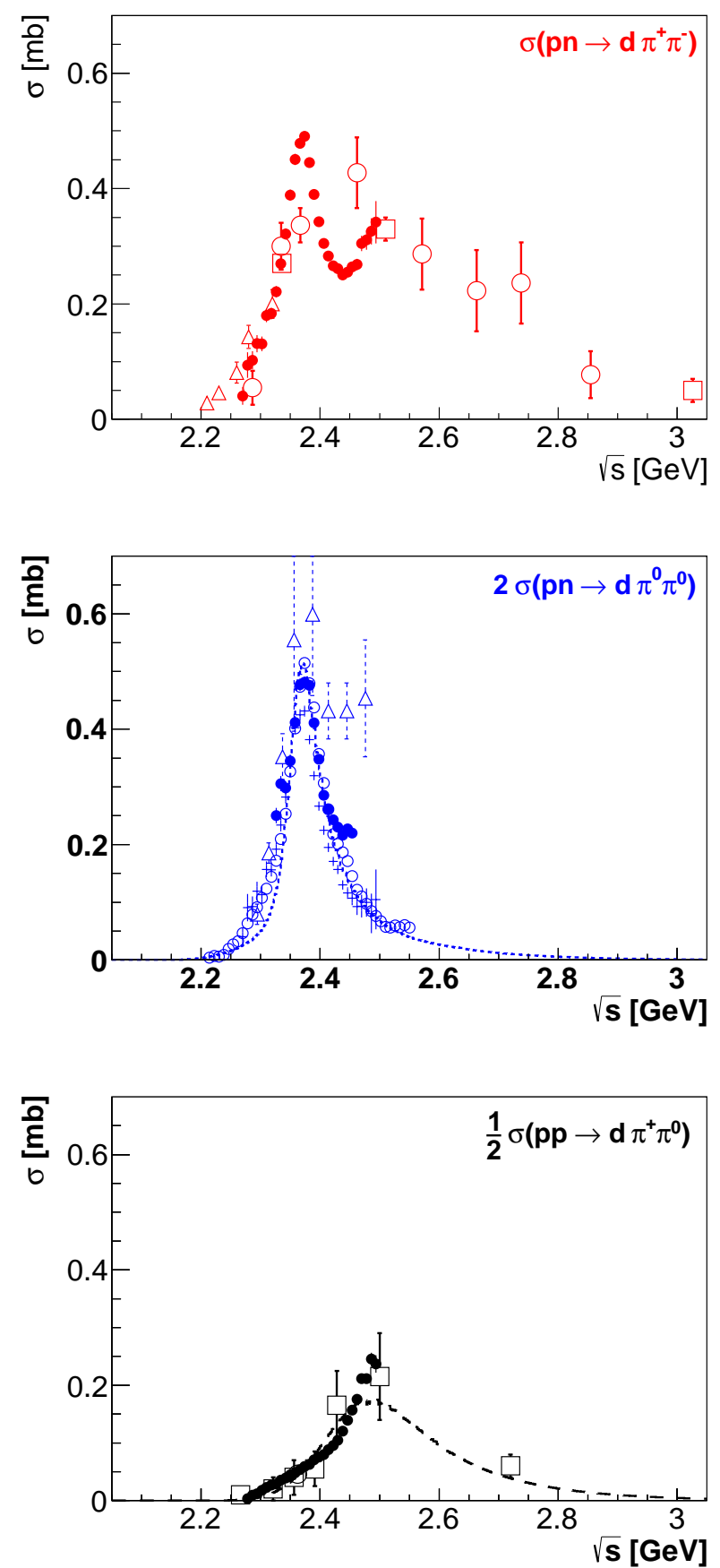

Figure 12: Total cross sections of the double-pionic fusion reactions to deuterium and their isospin decomposition. Top: the isospin-mixed reaction $p n \rightarrow d \pi^{+} \pi^{-}$. Solid dots represent WASA-at-COSY measurements, open symbols previous bubble-chamber measurements at DESY (circles) [358], Dubna (squares) [359] and Gatchina (triangles) [317]. Middle: the isoscalar reaction $p n \rightarrow d \pi^{0} \pi^{0}$ plotted as the isoscalar part of the $p n \rightarrow d \pi^{+} \pi^{-}$reaction. The CELSIUS/WASA results [27] are shown by open triangles. The other symbols refer to WASA-at-COSY measurements [26, 28]. The dotted line gives a Lorentzian with $\mathrm{m}=2380 \mathrm{MeV}$ and $\Gamma=70 \mathrm{MeV}$. Bottom: the isovector reaction $p p \rightarrow d \pi^{+} \pi^{0}$ plotted as the isovector part of the $p n \rightarrow d \pi^{+} \pi^{-}$reaction. Solid dots represent WASA-at-COSY results, open symbols previous results from [319, 328] as well as those given in Ref. [333]. The dashed line shows a $t$-channel $\Delta \Delta$ calculation fitted in height to the data [328]. From [28]. 
[336] - we may calculate the contribution of this process to the other double-pionic fusion channels. In the $p n \rightarrow d \pi^{+} \pi^{-}$reaction this process should contribute with $9 / 10$ of the strength observed in the $p p \rightarrow d \pi^{+} \pi^{0}$ reaction, i.e., this process is expected to provide about $0.32 \mathrm{mb}$ at the peak around 2.5 $\mathrm{GeV}$ in the total cross section. We see that this process accounts reasonably well for the measured data beyond $\sqrt{s}=2.4 \mathrm{GeV}$.

For the $p n \rightarrow d \pi^{0} \pi^{0}$ reaction isospin decomposition of the conventional $\Delta \Delta$ process implies that only $1 / 5$ of the strength observed in the $p p \rightarrow d \pi^{+} \pi^{0}$ reaction is to be present in this channel, which gives a peak contribution of $0.06 \mathrm{mb}$ around $2.5 \mathrm{GeV}$. This value is small compared to the value of $0.28 \mathrm{mb}$ observed at the peak of the $d^{*}$ resonance structure and explains, why this channel is the golden channel with regard to a small background from conventional processes. We note in passing that the contribution from the $t$-channel Roper excitation is still much smaller [363].

\subsection{Search for the $d^{*}$ Resonance Structure in Isoscalar Non-Fusion Two-Pion Pro- duction}

Recently also the non-fusion two-pion production channels $p n \rightarrow p p \pi^{0} \pi^{-}[29], p n \rightarrow p n \pi^{0} \pi^{0}$ [30], $p n \rightarrow p n \pi^{+} \pi^{-}$360, which are partially isoscalar, have been investigated. The WASA results (solid circles) together with previous data (open symbols) as well as a recent data point (solid triangle) from HADES [366] are shown for the total cross sections in Fig. 13. Since the four-body phase space grows much more rapidly with increasing energy than the three-body phase space does, also the contribution of the conventional $t$-channel processes grows rapidly with energy. In addition, since these reactions are only partially isoscalar, the amount of the conventional processes relative to the expected isoscalar $d^{*}$ contributions is much bigger. Hence the $d^{*}$ signal is likely to appear only as a kind of shoulder within the steeply increasing slope of total cross sections.

The size of the $d^{*}$ contribution can be easily estimated by use of the isospin-decomposition of twopion production [317, 333], where the $d^{*}$ contribution enters only in the reduced matrix elements $M_{000}$ and $M_{110}$ introduced in section 8.4. Having the $d^{*}$ decaying via an intermediate $\Delta \Delta$ system we get in addition that $M_{000}^{\Delta \Delta}=\sqrt{2} M_{110}^{\Delta \Delta}$ using isospin recoupling by means of $9 j$ symbols as described in Ref. 336] and as given in eq. (3). A more detailed treatment takes into account also the different phase-space situation, when the deuteron is replaced by the unbound pn system [364, 365].

For the $p n \rightarrow p n \pi^{+} \pi^{-}$reaction the data base is still very limited, since it has not been investigated by WASA. Hence, so far only bubble-chamber data are available for this channel - with the exception of new data point from a recent HADES measurement at $2.43 \mathrm{GeV}$ [366]. Further measurements in the $d^{*}$ energy region are expected to be performed by HADES.

As as result all two-pion production channels are consistent with the hypothesis of an $I\left(J^{P}\right)=0\left(3^{+}\right)$ dibaryon resonance at $2.37 \mathrm{GeV}$ with a width of $70 \mathrm{MeV}$. Though these reactions are only partially isoscalar, the $d^{*}$ contributions, which are shown by the dashed lines in Fig. 13 , constitute still the by far dominating process in the $d^{*}$ energy region. There the conventional $t$-channel processes (solid lines) underpredict the data by factors two to four.

The observed $d^{*}$ decay branchings into the diverse two-pion channels are consistent with expectations from isospin decomposition [367] as well as explicit theoretical calculations [364, 365] - as will be discussed in detail in section 10.4 .

\section{$10 d^{*}(2380)$ - a Genuine Dibaryon Resonance}

In order to prove that the resonance structure observed in two-pion production indeed constitutes a true resonance, i.e. a $s$-channel resonance, it has to be sensed also in the entrance channel, i.e. in 

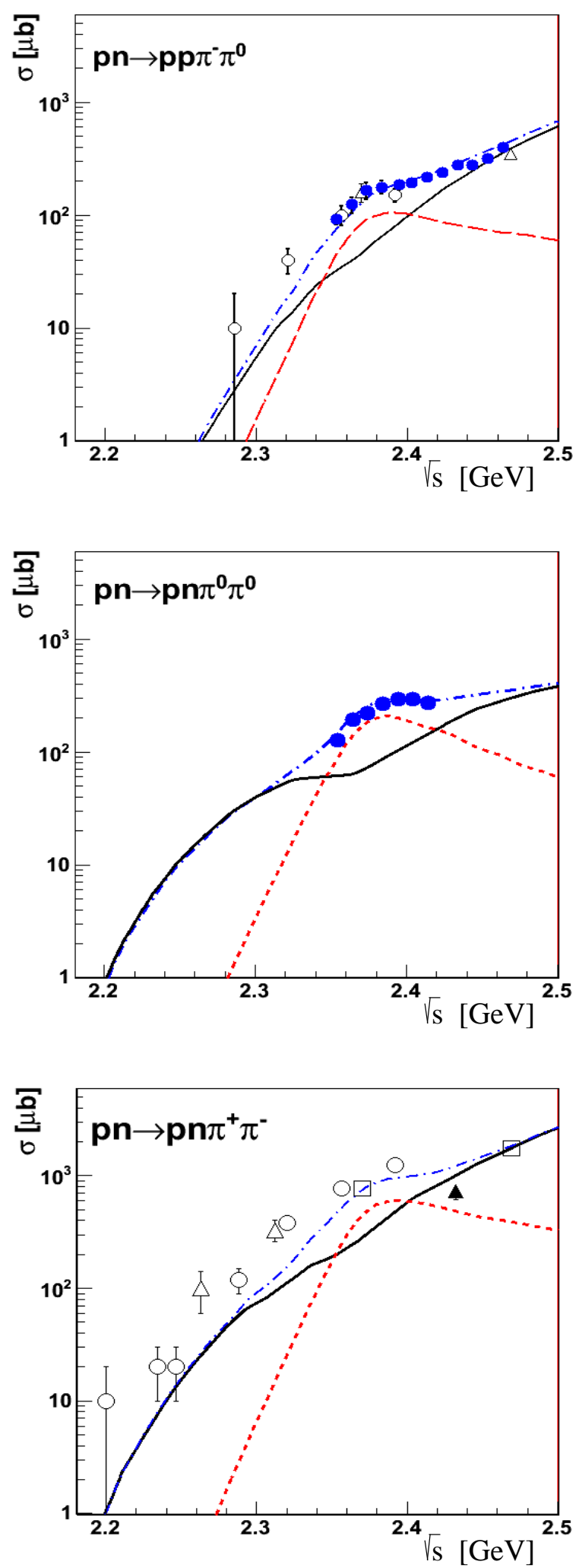

Figure 13: Total cross sections of the two-pion production reactions, where the two nucleons do not fuse and where there is an isoscalar reaction component, namely the reactions $p n \rightarrow p p \pi^{0} \pi^{-}$(top), $p n \rightarrow$ $p n \pi^{0} \pi^{0}$ (middle) and $p n \rightarrow p n \pi^{+} \pi^{-}$(bottom). Solid dots represent WASA-at-COSY measurements, open symbols data from previous measurements [318, 356, 359]. The solid triangle denotes a recent results from HADES [366]. Solid, dotted and dash-dotted lines show the contributions from conventional $t$-channel meson-exchange, the $d^{*}(2380)$ resonance and their coherent sum, respectively. 
$n p$ scattering. There it has to be shown that it produces a pole in the partial waves corresponding to $I\left(J^{P}\right)=0\left(3^{+}\right)$, i.e. in the coupled partial-wave system ${ }^{3} D_{3}-{ }^{3} G_{3}$.

From the knowledge of the resonance contribution to the two-pion channels the expected resonance contribution to elastic $n p$ scattering can be estimated [367] to be in the order of about $170 \mu \mathrm{b}$, which has to be compared to a total $n p$ cross section of nearly $40 \mathrm{mb}$. The only easily accessible observable, which has the potential to sense such a small contribution, is the analysing power, since it is composed of only interference terms in the partial waves and hence sensitive to small contributions in partial waves. Due to the spin of the resonance its angular contribution in the analysing power has to be according to the angular dependence of the associated Legendre polynomial $P_{3}^{1}$. Hence the resonance contribution is expected to be largest at $90^{\circ}$, i.e., at the angle, where the differential cross section is smallest. For the sensitivity of other observables to the resonance contribution see Ref. [368]

Following these considerations the analyzing power was measured over the energy region of interest and over practically the full angular range with WASA at COSY. The experiment was carried out again in the quasi-free mode, but in inverse kinematics by use of a polarized deuteron beam hitting the hydrogen pellet target.

Fig. 14 shows the results of the measurements and their subsequent partial-wave analysis by the SAID group [31, 32, 368, 369, 370]. The top panel displays the energy dependence of the analysing power in the region of $90^{\circ}$, where the effect of the resonance contribution is expected to be largest. The new WASA data (solid circles) exhibit a resonance-like structure right at the position, where expected and where no data were available from previous measurements (open symbols) [371, 372, 373, 374, 375, 376, 377, 378. The solid lines shows the previous SAID partial-wave solution SP07 [304], which completely misses the new data. The dashed line exhibits the new SAID solution SM16 [370] upon inclusion of the new WASA data.

The middle panel shows the angular distribution of the analysing power right at resonance. Clearly, the old SP07 solution misses the data over most of the angular range, but most dramatically around $90^{\circ}$, where $P_{3}^{1}$ is largest. The only agreement between old and new solutions is at $63^{\circ}$ and $116^{\circ}$, the zeros of $P_{3}^{1}$.

The bottom panel displays the Argand diagram of the new solution for the ${ }^{3} D_{3}$ partial wave. It exhibits a pronounced looping of this partial wave in agreement with a resonant behavior. The new partial-wave analysis SM16 reveals, indeed, a pole in the coupled ${ }^{3} D_{3}-{ }^{3} G_{3}$ partial waves at the position $(2380 \pm 10)-i(40 \pm 5) \mathrm{MeV}$ [31, 32, 368, 369, 370] - fully consistent with the findings in the two-pion production reactions.

This result establishes the resonance structure observed in two-pion production as a true $s$-channel resonance in the proton-neutron system. Since it is of isoscalar character, $d^{*}(2380)$ has been chosen as its denotation in analogy to the notation for isoscalar excitations of the nucleon. Also, the notation $d^{*}$ was used already by Goldman et.al. [34] when predicting the so-called "inevitable dibaryon" with identical quantum numbers.

When investigating the genuineness of a resonance the important question arises, whether there are nearby thresholds, which possibly could simulate a resonance effect in form of cusp due to channelcoupling. Indeed, at $\sqrt{s}=2.38 \mathrm{GeV}$ there are two thresholds, the $N N \eta$ and the $N N^{*}(1440)$ production thresholds. Both processes are both of isoscalar and isovector character and hence any hypothetical cusp effect would necessarily be observed also in $p p$-induced two-pion production - which, however is not the case. This disproves also the argumentation of Bugg in Ref. [357], who suggests the $d^{*}(2380)$ resonance structure to be the result of the $N^{*}(1440)$ excitation. As discussed above already in sec. 8.4 this excitation with a width of about $300 \mathrm{MeV}$ is well understood and contributes only as a marginal, very broad background process in the golden channel $p n \rightarrow d \pi^{0} \pi^{0}$. 

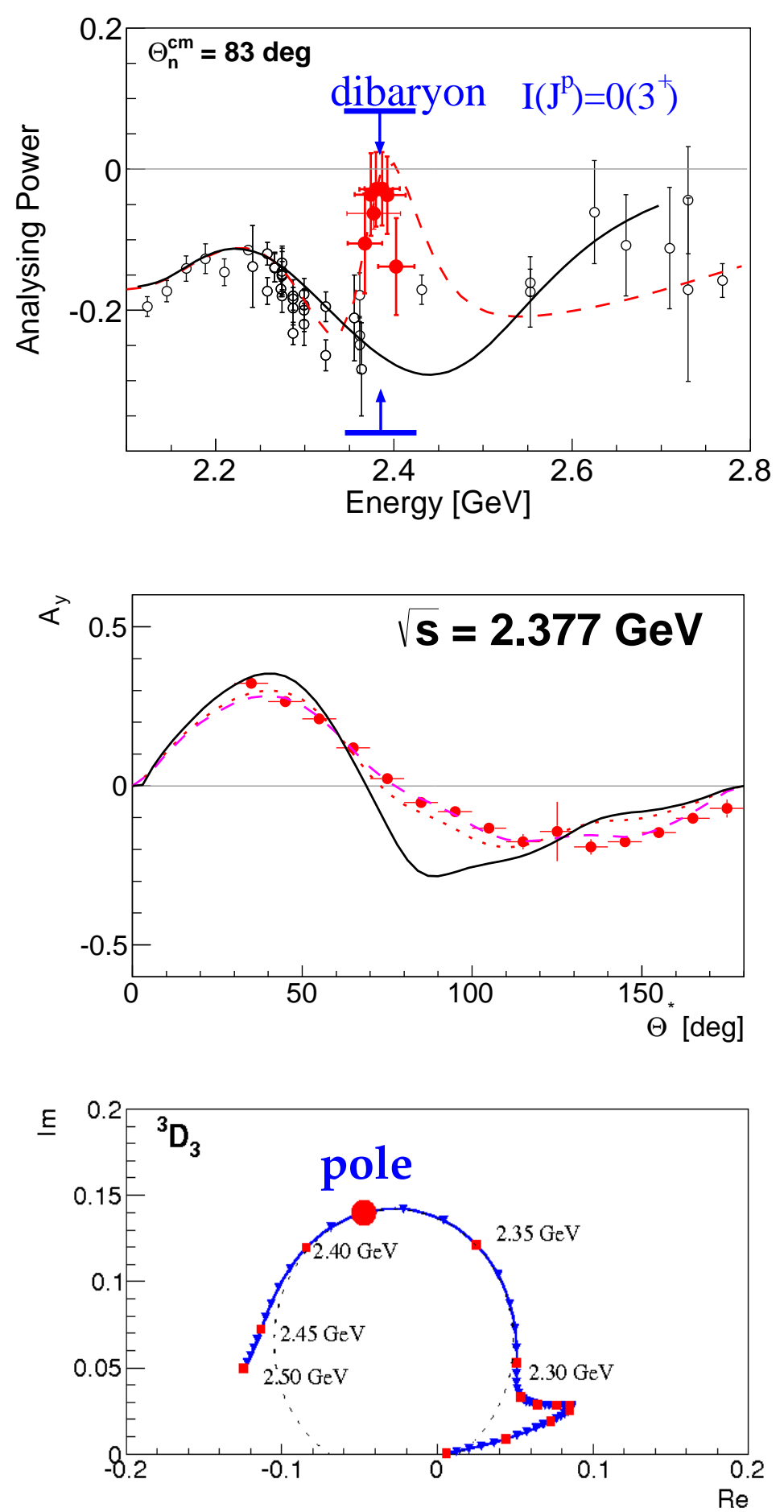

Figure 14: Analysing power data for elastic $p n$ scattering in the $d^{*}(2380)$ energy region and their partialwave analysis [31, 32, 368, 369, 370]. Top: energy dependence of the analysing power in the vicinity of $\Theta_{n}^{c m}=90^{\circ}$, where the effect of the $d^{*}(2380)$ resonance is expected to be largest. The solid circles denote WASA results, the open symbols previous data [371, 372, 373, 374, 375, 376, 377, 378, The solid line gives the previous SAID partial-wave solution, the dashed line the new SAID solution after including the WASA data. Middle: Angular distribution of the analysing power $A_{y}$ at the resonance energy. The curves have the same meaning as in the top figure. Bottom: Argand diagram of the new SAID solution for the ${ }^{3} D_{3}$ partial wave with a pole at $2380 \mathrm{MeV}$. The thick solid circle denotes the pole position. 


\subsection{Former Occasions for Its Discovery}

Having established now $d^{*}(2380)$ as a genuine dibaryon resonance we may ask the question, whether already previous experiments could have had the chance to discover it. As already mentioned above, the golden channel $p n \rightarrow d \pi^{0} \pi^{0}$ was not accessible instrumentally before. But what about other channels?

In the $p n \rightarrow d \pi^{+} \pi^{-}$reaction the resonance effect is no longer as pronounced, but still could have been observed in principle by previous bubble chamber experiments at DESY [358] and JINR [359] in case of much improved statistics as well as better neutron beam energy resolution and a careful energy scan, respectively.

Previous $n p$ scattering experiments at LAMPF and Saclay were limited by the maximum beam energy available at these accelerator facilities, which were below the energy region of interest here. In this respect it was particularly unfortunate that the maximum available beam energy at Saclay marginally missed the $d^{*}(2380)$ resonance region.

The EDDA experiment at COSY would have had a great chance of its discovery, if its $p p$ scattering program would have been complimented by a corresponding $n p$ scattering program - as intended initially.

Actually groups at Tokyo had been quite close already as early as 1977 considering measurements of the proton polarization in deuteron photodisintegration $\gamma d \rightarrow \overrightarrow{p n}$ [129, 130, 131, 132]. They observed a strong increase of the polarization at energies beyond $\sqrt{s}=2.2 \mathrm{GeV}$, reaching a maximum around $2.38 \mathrm{GeV}$ and decreasing thereafter. From the fact that the effect appeared to be largest at $90^{\circ}$ they concluded that possibly an $I\left(J^{P}\right)=0\left(3^{+}\right)$deeply bound $\Delta \Delta$ system could be the reason - making also a possible connection to $\mathrm{ABC}$ effect and two-pion production channels. Only, their data favored a broad resonance of about $160 \mathrm{MeV}$ width. Follow-up measurements and their analysis showed that the data and their analysis are best described by the assumption of several broad resonances of different spin-parity with widths larger than $200 \mathrm{MeV}$ [132]. From the large widths it looks that these findings correspond to $t$-channel $\Delta \Delta$ excitations rather than the narrow $d^{*}(2380)$ dibaryon resonance. However, we note that recent JLAB measurements of the proton polarization at $90^{\circ}$ [379] confirm a maximum polarization of -1 at $\sqrt{s} \approx 2.38 \mathrm{GeV}$ with a sharp decrease at higher energies. This indicates that on top of polarization effects due to conventional $t$-channel $\Delta \Delta$ excitations there might be, indeed, a visible effect from $d^{*}(2380)$. If true, it would open the door to measurements of the electromagnetic excitation of $d^{*}(2380)$ in such reactions providing access to the formfactor and thus to the size of this object.

\section{$10.2 d^{*}(2380)$ in Nuclear Matter}

If dibaryons exist and if they even survive in a nuclear surrounding, then they should have an impact on the nuclear equation of state [380, 381, 382, 383, which is of relevance not only for heavy-ion collisions but also for very compact stellar objects like neutron stars. The effect of $\Delta$ and other baryon excitations on the equation of states has been considered also in Ref. [384.

As already expected from the ABC effect observed previously in the double-pionic fusion reactions to the He isotopes, the exclusive and kinematically complete measurements of these fusion reactions conducted with WASA at COSY could demonstrate [385, 386] that indeed $d^{*}(2380)$ is present also in these reactions albeit with a much increased width due to the Fermi motion of the nucleons bound in these nuclei.

To investigate this question for still heavier nuclei by exclusive double-pionic fusion reactions becomes very difficult from the experimental point of view, since the kinetic energies of the fusion products get such low that they get below the detection thresholds of traditional experimental setups and would afford new, very sophisticated instruments. A way out would be measurements with inverse kinematics, e.g., using a ${ }^{14} \mathrm{~N}$ beam to conduct the reaction ${ }^{14} \mathrm{~N} d \rightarrow{ }^{16} \mathrm{O} \pi^{0} \pi^{0}$.

Support for the existence of $d^{*}(2380)$ in nuclear matter may come from heavy-ion collisions in 
connection with the so-called DLS puzzle. In relativistic heavy-ion collisions an unusual enhancement has been observed in the spectrum of emitted $e^{+} e^{-}$-pairs with invariant masses between $\pi$ and $\omega$ mass. First interpreted as a possible signal from the quark-gluon plasma it was soon realized that this enhancement persists also at low collision energies. Dedicated measurements of dilepton production in nucleon-nucleon collisions by DLS [387] and HADES [388] could trace back this phenomenon to a not-understood enhancement in $p n$ collisions at energies below $\sqrt{s} \approx 3 \mathrm{GeV}$.

In a recent publication [336] it could be demonstrated that two-pion production via $t$-channel $\Delta \Delta$ excitation as well as via $d^{*}(2380)$ formation - where the produced $\rho$-channel pion pair transforms into a lepton pair - can account quantitatively for this enhancement and thus solve the DLS-puzzle. Since this enhancement is observed also in heavy-ion collisions, this means in turn that obviously also $d^{*}(2380)$ has been produced in those collisions.

\section{$10.3 d^{*}(2380)$ in Theory}

As already discussed in section 3 the very first theoretical paper on dibaryons in connection with quarks - or more precise with SU(6) symmetry breaking - the one by Dyson and Xuong in 1964 [33], predicted properly the now discovered state $d^{*}(2380)$ at a mass remarkably close to the experimentally observed one. Subsequent, partially very complex theoretical investigations, turned out to be much less successful. In particular they predicted often a multitude of states in addition, which were never observed.

In connection with the observation of large polarization effects in deuteron photodisintegration (see Sect. 10.1) also Kamae and Fujita [99] predicted 1977 the mass of the now observed state properly based on a non-relativistic one-boson exchange potential model for two $\Delta$ isobars.

Goldman et al. 34 pointed out 1989 that due to the unique symmetries of such a state any model based on confinement and effective one-gluon exchange must predict the existence of this state - calling it the "inevitable" dibaryon. However, initially they predicted an enormous binding energy as large as $350 \mathrm{MeV}$ for such a state. Only recently - after the experimental observation - the calculations of this group approached the experimental value in the framework of the so-called quark delocalization and color screening model (QDCSM) [389, 37].

Also the Nijmegen group [75, 76, 77, 78] as well as Mulders and Thomas [79] and also Saito [80] predicted this state correctly at the proper mass in their bag-model calculations. Only, they simultaneously predicted within the same framework numerous other unflavored dibaryon states, which were never observed.

A correct real prediction, i.e. a prediction before the experimental discovery of $d^{*}(2380)$, is the one by the IHEP theory group led by Z. Y. Zhang, who studied this state in the chiral $S U(3)$ quark model within the framework of the resonating group method (RGM) [38].

Meanwhile, also a QCD sum rule study finds this state at the right mass [390]. On the other hand, a study purely based on quarks and gluons does not obtain this state, at least not in the energy region of the experimental observation [391]. The reason might be that no coupling to hadrons, where $d^{*}(2380)$ finally has to decay into, has been taken into account. A first result from lattice QCD has been reported at the MPMBI 2015 workshop at Sendai by K. Sasaki from the HALQCD collaboration [392]. For the $\Delta \Delta$ system with $J=3$ a strongly attractive interaction has been obtained, which leads to a bound state - albeit these calculations with a pion mass of $1015 \mathrm{MeV}$ are still far away from being very realistic.

A relativistic Faddeev-type calculation based on hadronic interactions have been reported by Gal and Garcilazo recently to see this state, too, correctly at the experimental mass [9, 35, 36]. Already before that Garcilazo et al. had predicted this state based on two-body interactions derived from the chiral quark cluster model, though with too small a binding energy of only $29.9 \mathrm{MeV}$ [393, 394] and $7.8 \mathrm{MeV}$ [205], respectively. However, those calculations were performed without coupling to the $N N$ channel, which would lead to larger binding energies. It should be noted that those calculations give the same sequence of dibaryon states as predicted previously by Dyson and Xuong [33]. 
More demanding than the mass value appears to be the reproduction of the decay width of $d^{*}(2380)$, since it requires also a theoretical treatment of the dynamics of the decay process. So far there are only three theoretical predictions for the decay width based either on Faddeev calculations [9, 35, 36] or on quark-model calculations within the QDCSM framework [389, 37] and the RGM-based chiral $S U(3)$ model [395, 396, 397], respectively.

In the SAID partial-wave analysis [370] the generated pole has an imaginary part of $40 \mathrm{MeV}$, which in a Breit-Wigner interpretation corresponds to a width of $80 \mathrm{MeV}$. Actually the analysing power data for the energy dependence around $90^{\circ}$ shown in the top panel of Fig. 14 would prefer a somewhat narrower width, but the data base at the high-energy end of the resonance region is too sparse for a more stringent fixation of the width from $p n$ scattering. Much better is the data base in two-pion production, in particular in the golden channel $p n \rightarrow d \pi^{0} \pi^{0}$, where the Lorentzian shape of the energy dependence of the total cross section is well documented by high-statistics data, see top panel in Fig 11 . These data prefer a width of $70 \mathrm{MeV}$. Though the background due to conventional processes, essentially $t$-channel $\Delta \Delta$ excitation, is small, it introduces a small uncertainty in the exact determination of the width here, too.

From the calculation of the $t$-channel $\Delta \Delta$ process we know that the plain $\Delta \Delta$ excitation leads to a resonance structure with a width of about twice the $\Delta$ width 2 i.e. $230 \mathrm{MeV}$ - see, e.g. Fig. 12 , bottom. If we consider now the asymptotic configuration of $d^{*}(2380)$ as a $\Delta \Delta$ system bound by $80 \mathrm{MeV}$, then we may easily estimate its decay width by the known momentum dependence of the $\Delta$ decay width, which is proportional to the third power of the effective decay momentum, see, e.g., Ref. [349]. With having a $\Delta$ mass reduced by $40 \mathrm{MeV}$ due to binding this leads to a reduced width of about $160 \mathrm{MeV}$ for a $\Delta \Delta$ system bound by $80 \mathrm{MeV}$ [11]. This is also the result of Ref. [389] for the $d^{*}(2380)$ decay width. In a more detailed treatment taking into account correlations the QDCSM calculations arrive at a width of $110 \mathrm{MeV}$ [37]. Also, if we calculate the $d^{*}$ width by use of eq. (12) in Ref. [362], then we obtain a width of $107 \mathrm{MeV}$ at the $d^{*}$ mass starting from a width of $230 \mathrm{MeV}$ for the unbound $\Delta \Delta$ system.

The Faddeev calculations come closer to the experimental value by obtaining a width of $94 \mathrm{MeV}$ for a resonance mass of $2383 \mathrm{MeV}[9,35,36]^{3}$. To this the decay width into the $p n$ system has to be added, so that a total decay width of slightly above $100 \mathrm{MeV}$ is obtained.

Common to these theoretical investigations is that they overestimate the width of $d^{*}(2380)$. This may possibly be related to exotic contributions to this state - like, e.g., hidden color aspects as discussed in Ref. [11]. In fact the calculations of the IHEP group, which include hidden color configurations arrive at a width of $72 \mathrm{MeV}$ [395, 396, 397] in full agreement with the experimental result. In these calculations the $d^{*}(2380)$ wave function contains about $67 \%$ hidden color components, which can not decay easily and hence reduce the decay width to the experimental value. Also the Nanjing group finds a reduction of $25 \mathrm{MeV}$ in the decay width, if coupling to hidden color channels is taken into account [37]. Hiddencolor configurations for a hexaquark state with the quantum numbers of $d^{*}(2380)$ have been investigated recently also in Ref. 399.

These studies suggest that the observed unusually small decay width of $d^{*}(2380)$ signals an exotic character of this dibaryon resonance and points to a compact hexaquark nature of this object as discussed in detail in Refs. [396, 39, 400]. These calculations obtain a root-mean-square radius of 0.8 fm for the size of this object. Also the Nanjing calculations give similar results for the size of $d^{*}(2380)$. If true then this resonance indeed constitutes a very compact hexaquark system. Reservations about the compact hexaquark interpretation of $d^{*}(2380)$ have been voiced recently by Gal [401].

\footnotetext{
${ }^{2}$ We note that in a recent article [398] it is argued that the width of an unbound $\Delta \Delta$ configuration is equal to the width of just a single $\Delta$.

${ }^{3}$ The solution with a factor $2 / 3$ applied assumes that there is no decay into $p p \pi^{0} \pi^{-}$and $n n \pi^{+} \pi^{0}$ channels as well as a reduced decay into the $n p \pi^{+} \pi^{-}$channel, which is not in accordance with experimental results, see discussion of branchings in the next section
} 
Table 2: Branching ratios in percent of the $d^{*}(2380)$ decay into $p n, N N \pi$ and $N N \pi \pi$ channels. The experimental results [367, 402] are compared to results from a theoretical calculation [397] starting from the theoretical $d^{*}$ wave function and including isospin breaking effects. They are also compared to values expected from pure isospin recoupling of the various $N N \pi \pi$ channels according to eqs. (3) and (5), respectively. In the latter case the branching into the $d \pi^{0} \pi^{0}$ channel is normalized to the data.

\begin{tabular}{lccc}
\hline & & & \\
decay channel & experiment & theory 397 & $N N \pi \pi$ isospin recoupling \\
\hline & & & \\
$d \pi^{0} \pi^{0}$ & $14 \pm 1$ & 12.8 & 13 \\
$d \pi^{+} \pi^{-}$ & $23 \pm 2$ & 23.4 & 26 \\
$n p \pi^{0} \pi^{0}$ & $12 \pm 2$ & 13.3 & 13 \\
$n p \pi^{+} \pi^{-}$ & $30 \pm 5$ & 28.6 & 32.5 \\
$p p \pi^{0} \pi^{-}$ & $6 \pm 1$ & 4.9 & 6.5 \\
$n n \pi^{+} \pi^{0}$ & $6 \pm 1$ & 4.9 & 6.5 \\
$(N N \pi)_{I=0}$ & $<9$ & 0 & - \\
$n p$ & $12 \pm 3$ & 12.1 & - \\
\hline & & & \\
$\sum($ total $)$ & $103 \pm 7$ & 100 & \\
\hline
\end{tabular}

\subsection{The Branchings of the $d^{*}(2380)$ Decays}

The decays of $d^{*}(2380)$ into $p n$ and $N N \pi \pi$ channels derive from the analysis of $p n$ elastic scattering and the measurements of the $d^{*}(2380)$ signal in the various two-pion production reaction channels. The branching ratios into these channels have been evaluated from the data in Ref. [367] and are presented in Table 2.

A hypothetical decay $d^{*}(2380) \rightarrow N N \pi$ has been investigated by analyzing WASA data for the single-pion production reactions $p p \rightarrow p p \pi^{0}$ and $n p \rightarrow p p \pi^{-}$, in order to extract the isoscalar singlepion production cross section in the energy region of the $d^{*}(2380)$ resonance. The resulting data on the isoscalar single-pion production give no evidence for a sizeable contribution from the $d^{*}(2380)$ decay into the isoscalar $N N \pi$ channel [402].

As already discussed in sections 8.4, 9.2 and 9.3 the cross sections of the various two-pion production channels are closely linked by isospin relations [317, 333, 336], where the reduced matrix elements $M_{I_{N N}^{f} I_{\pi \pi} I_{N N}^{i}}$ enter. For formation and decay of $d^{*}(2380)$ only the matrix elements $M_{000}$ and $M_{110}$ are of relevance.

In the following we discuss, how these branchings are sensitive to the intermediate asymptotic hadronic configuration, where $d^{*}(2380)$ decays to. Whereas Dyson and Xuong [33] as well as QCDbased quark models [75, 76, 77, 78, 179, 80, 34, 389, 37, 38, 395, 396, 397] predict a $\Delta \Delta$ system as hadronic intermediate configuration, the work of Kukulin and Platonova [206, 361, as well as that of Gal and Garcilazo [35, 36] prefer a $D_{12} \pi$ intermediate configuration, where the $\Delta N$ threshold state $D_{12}$ decays subsequently into $N N$ and $N N \pi$ final states.

\subsection{1 compatibility with an asymptotic $\Delta \Delta$ configuration}

For an intermediate $\Delta \Delta$ system we have $M_{000}^{\Delta \Delta}=-\sqrt{2} M_{110}^{\Delta \Delta}$ from eq. (3). If we put this relation into the expressions of reduced matrix elements for the various two-pion cross sections, then we obtain the branchings into the various $N N \pi \pi$ channels as given in the fourth column of Table 2, As discussed 
in Ref. [28] the isospin breaking due to mass differences between neutral and charged pions as well as between proton and neutron - which entail differences in $N N \pi \pi$ reaction thresholds - causes changes in these values in the order of $10-20 \%$. The same is true for the comparison of $d \pi \pi$ and unbound $N N \pi \pi$ channels due to different phase spaces as treated in more detail in Refs. [364, 365]. With this in mind we find excellent agreement between the experimentally determined branchings and the ones expected for the case that $d^{*}(2380)$ decays into an intermediate $\Delta \Delta$ system. The branching into the pn system is then obtained by using unitarity as detailed in Ref. [367]. As also discussed in that work there is no known process, which could feed the isoscalar $N N \pi$ channel starting from an intermediate isoscalar $\Delta \Delta$ configuration.

In Ref. [397] the branching has been calculated starting directly from the theoretical $d^{*}$ wave function obtained in a chiral $S U(3)$ quark model calculation. Asymptotically this wave function represents a bound $\Delta \Delta$ configuration containing hidden color. This calculation, which also includes isospin breaking effects due to different masses of charged and neutral pions and baryons, is shown in the third column of Table 2, It is in excellent agreement with the experimental results given in the second column of Table 2 ,

\subsection{2 compatibility with a decay route via $D_{12}$ ?}

Kukulin and Platonova [206, 361] propose as an alternative to an asymptotic $\Delta \Delta$ configuration the decay of $d^{*}(2380)$ via the $\Delta N$ threshold state $D_{12}$ by emission of a $p$-wave pion. They argue that due to the small size of the compact six-quark object $d^{*}(2380)$ it is much more natural for it to decay into another small six-quark object, $D_{12}$, by pion emission rather than into a much more extended $\Delta \Delta$ system.

Gal and Garcilazo [35, 36] have taken up this idea, since it provides the possibility to reduce the fourbody $N N \pi \pi$ system effectively to a three-body $\Delta N \pi$ system with an intermediate $D_{12} \pi$ configuration, which they then can handle in the Faddeev formalism.

Indeed, a $D_{12} \pi$ configuration with the pion in relative $p$-wave is kinematically hardly to distinguish from a $\Delta \Delta$ configuration. The Dalitz plot would look the same. So the only way to check this alternative hypothesis appears to be the branchings of the $d^{*}(2380)$ decay, which contain also the dynamics and structure informations.

Assuming again isospin invariance we may obtain the branching for the $D_{12} \pi$ scenario simply by considering the decays of $D_{12}$ into the various $N N \pi$ channels. By isospin recoupling we obtain then 401 .

$$
M_{I_{N N}^{f} I_{\pi \pi} I_{N N}^{i}}^{D_{12} \pi_{2}} \sim(-)^{I_{N N}^{f}} \hat{I}_{N N}^{f} \hat{I}_{\Delta}\left\{\begin{array}{ccc}
I_{N_{1}} & I_{N_{2}} & I_{N N}^{f} \\
I_{D_{12}} & I_{\pi_{1}} & I_{\Delta}
\end{array}\right\}
$$

where the $N N$ system in the final state couples with $\pi_{1}$ to $D_{12}$ and further-on with $\pi_{2}$ to $d^{*}(2380)$.

Of relevance for the branchings into the various $N N \pi \pi$ channels are again only the matrix elements $M_{000}$ and $M_{110}$, which according to eq. (5) are related by $M_{000}=-\sqrt{2} M_{110}$. Since the cross sections depend just on these matrix elements squared, we obtain identical branchings into the various $N N \pi \pi$ channels for both scenarios.

Besides of the decays of $D_{12}$ into the isovector $N N \pi$ channels, $D_{12}$ decays also into the isovector $N N$ system. Hence $d^{*}(2380)$ decays also into the isoscalar $N N \pi$ channel in this scenario via the route $d^{*}(2380) \rightarrow\left[D_{12} \pi\right]_{I=0} \rightarrow[N N \pi]_{I=0}$. The SAID partial-wave analyses [189, 304, 370] of elastic $p p$ scattering give a modulus of the residue at the $D_{12}$ pole of $\mathrm{G}=10-11 \mathrm{MeV}$, which results in a branching of the $D_{12} \rightarrow N N$ decay of $16-18 \%$. The isoscalar single-pion production has been analyzed at WASA with the aim to search experimentally for a possible decay branch $d^{*}(2380) \rightarrow[N N \pi]_{I=0}$. No 
indication in the data for such a decay has been found, only an upper limit of $9 \%$ (90\% C. L.) could be derived 402 .

Note that different from the case with a $\Delta \Delta$ intermediate state there is no decay route into the $n p$ system in this scenario with an intermediate $D_{12} \pi$ configuration. Hence the decay route into the $n p$ channel, which has been measured to have a branching of $12 \pm 3 \%$, has to be added by hand in this scenario.

In the model of Gal and Garcilazo [35, 36] only decay modes with $I_{N N}=0$ are allowed in leading approximation, i.e. there are no $I_{N N}=1$ contributions of the decays into the $p p \pi^{0} \pi^{-}, n n \pi^{+} \pi^{0}$ and $p n \rightarrow p n \pi^{+} \pi^{-}$channels. In a forthcoming paper [401] various possibilities are discussed, how agreement can be achieved between experimental branching ratios and the ones obtained in a $D_{12} \pi$ scenario.

Summarizing, in both scenarios we find identical decay branchings into the various $N N \pi \pi$ channels. In the $D_{12} \pi$ scenario we expect a substantial decay into the isoscalar $N N \pi$ system with no decay into the $n p$ channel, whereas in the $\Delta \Delta$ scenario the situation is just opposite. However, their $D_{12}$ and therefore also $d^{*}(2380)$ are not compact six-quark objects.

\subsection{Size and Structure of $d^{*}(2380)$}

One of the most important questions with regard to $d^{*}(2380)$ is, whether it constitutes a large-sized molecule-like object or a compact hexaquark system. So far we do not have any conclusive answer to this from experiment. A possibility to approach this question experimentally will be discussed in the next section.

Also for the recently observed tetraquark and pentaquark systems there is as of yet no experimental knowledge about the size of these objects. Here the situation is even more confusing, since these objects have a very small decay width, though they have masses very close to adjacent thresholds and hence appear to be very loosely bound. In the theoretical discussion of these objects Piccinini et al. proposed [403, 404] a rough radius estimate for a system of two quark clusters based on the Heisenberg uncertainty relation and depending just on the binding energy $\epsilon$ of the object in question:

$$
r \approx \frac{\hbar c}{\sqrt{2 m \epsilon}}
$$

In case of the much-debated tetraquark system $X(3872)$, which is bound by only few keV, this leads to a radius of the order of $10 \mathrm{fm}$, which means a much extended molecular system. If we apply this formula to $d^{*}(2380)$ being bound by $80 \mathrm{MeV}$ relative to the nominal $\Delta \Delta$ threshold, then we obtain a radius of about $0.5 \mathrm{fm}$. Such a small radius means that the two quark clusters must strongly overlap and hence are expected to form a compact hexaquark system. This is also the conclusion obtained in detailed quark-model calculations, which give a radius of $0.8 \mathrm{fm}$ [396, 39, 400].

\subsection{1 electromagnetic excitation of $d^{*}(2380)$}

An electromagnetic excitation of the deuteron groundstate to the $d^{*}(2380)$ resonance is highly informative, since its transition formfactor gives access to size and structure of this resonance.

Judging just from the electromagnetic coupling constant, we expect electromagnetic decays to be suppressed by two order of magnitudes - as is borne out, e.g. in the decay of the $\Delta$ resonance. A technically feasible excitation of $d^{*}(2380)$ would start by photo- or electro-excitation from the deuteron groundstate. A real or virtual photon would need then to transfer two units of angular momentum, i.e. be of E2 or C2 multipolarity, which lowers the transition probability further. In addition, the overlap in the wavefunctions of $d$ and $d^{*}(2380)$ enters profoundly. We are aware of two theoretical calculations dealing with such a scenario [405, 406], where cross sections in the range $\mathrm{pb} / \mathrm{sr}$ - nb/sr are predicted for the forward angle region. This order of magnitude may be expected also by the simple consideration 
that the $d^{*}$ photo-production is facilitated by a double spinflip in the six-quark configuration of the deuteron. Since the latter is in the order of $10^{-3}$ - see section 2.1.1., the order of cross section, given by this number multiplied with the electromagnetic coupling constant, should be $10^{-5}$ that of the corresponding hadronic cross section [407]. I.e., since the peak cross section of the $p n \rightarrow d \pi^{0} \pi^{0}$ reaction is about $200 \mu \mathrm{b}$, the cross section of the $\gamma d \rightarrow d \pi^{0} \pi^{0}$ reaction should then be in the order of a few nanobarn.

The same order of magnitude is expected for the $d^{*}$ photoproduction in the $d \pi^{+} \pi^{-}$channel. But the $\gamma d \rightarrow d \pi^{+} \pi^{-}$cross section due to conventional processes is two orders of magnitude larger. Hence it appears very difficult to sense the resonance excitation under such conditions.

In this respect the reaction $\gamma d \rightarrow d \pi^{0} \pi^{0}$ appears to be attractive, since there the conventional processes are expected to be particularly small [408, 409]. In the energy region of $d^{*}(2380)$ the conventional cross section is predicted to be below $20 \mathrm{nb}$. In fact, first preliminary results from MAMI for this reaction [410] are in very good agreement with these predictions for incident photon energies $E_{\gamma}>600 \mathrm{MeV}$, where the calculations predict cross sections of 50 - $70 \mathrm{nb}$. Below that energy the calculations predict the cross section to be strongly decreasing, whereas the preliminary data show still appreciable strength, which possibly could be in accordance with a $d^{*}$ excitation [407]. Meanwhile also a measurement of this reaction at ELPH has been reported [411], which sees a similar energy dependence of the cross section, though its absolute value is larger by nearly a factor of two. Which of the two measurements is correct, has to be still evaluated.

Another way to sense such small contributions are polarization measurements. The situation looks similar to the one in elastic $n p$ scattering. As we have shown above, the $d^{*}(2380)$ resonance contribution is about $0.17 \mathrm{mb}$, which is more than two orders below the total elastic cross section. However, with help of the analyzing power, which consists only of interference terms in partial waves, it was possible to filter out reliably the resonance contribution.

The analogous case in electro- or photo-excitation of $d^{*}(2380)$ constitute measurements of the polarization of the outgoing proton in the reactions $\gamma d \rightarrow n \vec{p}$ and $\gamma^{*} d \rightarrow n \vec{p}$, respectively, where $\gamma^{*}$ stands for a virtual photon created in inelastic electron scattering on the deuteron. As in the analyzing power of $n p$ scattering the angular dependence of the resonance effect in the polarization of the outgoing proton should be proportional to the associated Legendre polynomial $P_{3}^{1}(\cos \Theta)$ [32]. Therefore, the maximal resonance effect is expected to be at a scattering angle of $\Theta=90^{\circ}$.

In fact, such an effect has already been looked for previously by Kamae et al. in corresponding data from the Tokyo electron synchrotron [129, 130, 131, 132]. In order to describe the observed large polarizations in the region of $d^{*}(2380)$, they fitted a number of resonances to the data, among others also a $J^{P}=3^{+}$state. However, presumably due to the limited data base they only obtained very large widths for these resonances in the order of $200-300 \mathrm{MeV}-$ as one would expect from conventional $\Delta \Delta$ excitations.

Recently, new polarization measurements from JLAB appeared [379]. Their lowest energy point is just in the $d^{*}(2380)$ region and is compatible with a maximal polarization of $P=-1$. It confirms thus the old Tokyo results in the sense that in this region there is a build-up of a very large polarization, which rapidly decreases both towards lower and higher energies, see Fig. 1 in Ref. [379]. Of course, a dedicated measurement over the region of interest is needed, in order to see, whether a narrow structure with the width of $d^{*}(2380)$ can be observed in this observable.

\subsection{2 the $A B C$ effect and the structure of $d^{*}(2380)$}

Coming back to the trace followed for the detection of $\mathrm{d}^{*}(2380)$ : the $\mathrm{ABC}$ effect. As demonstrated by the experiments, the ABC effect is strictly correlated with the occurrence of this resonance. But what is its explanation? In Ref. [26] ad hoc a vertex function for the decay vertex $d^{*}(2380) \rightarrow \Delta \Delta$ was introduced for its description. This vertex function parameterized in form of a monopole form-factor 
gives a good account for the data and with it for the $\mathrm{ABC}$ effect - see Fig. 11 -, if a cutoff parameter $\Lambda$ as small as $\Lambda=0.16 \mathrm{GeV} / \mathrm{c}$ is used. On a first glance this appears to be a surprisingly small value being three to four times smaller than usually used in the description of hadronic processes. However, the vertex functions used there are conventionally employed for $t$-channel meson exchanges. In our case of an $s$-channel resonance decay the assumed vertex function is actually identical to that used for the $\Delta \rightarrow N \pi$ decay with identical cutoff parameter [349, 362]. In fact, such vertex functions are commonly used for the description of the decay of baryon resonances [412] with cutoff parameters being similarly small as the value obtained for the $d^{*}(2380)$ case.

However, the unique situation in case of double-pionic fusion - where the momentum difference between nucleons cancels when neglecting the Fermi motion - is that the $\pi \pi$-invariant mass spectrum happens to map just the momentum dependence of the $d^{*}(2380) \rightarrow \Delta \Delta$ decay, which is given by that of the vertex function. Since this special kinematic condition holds no longer in case of non-fusion, this scenario also naturally explains the non-appearance of the ABC effect in non-fusion reactions. For a detailed recent discussion of this issue see Ref. [362], where also alternative concepts and other small contributions to the ABC effect are discussed.

Kukulin and Platonova [206, 361], e.g., proposed an alternative scenario for the $d^{*}(2380)$ decay and the $\mathrm{ABC}$ effect. Instead of the decay route $d^{*}(2380) \rightarrow \Delta \Delta$ they propose the dominating decay to be $d^{*}(2380) \rightarrow D_{12} \pi$ with a relative $p$-wave between $D_{12}$ and $\pi$ - as discussed in some detail already above in section 10.4.2. In addition to this route Kukulin and Platonova propose a $5 \%$ decay route $d^{*}(2380) \rightarrow d \sigma$ with a very light and narrow $\sigma$ meson in relative $d$-wave, in order to account for the ABC effect. Though there have been reports on the observation of narrow and/or light $\sigma$ mesons [413, 414, 415], a convincing clear-cut experimental confirmation is still missing. For the CELSIUS/WASA workshop contribution [15], e.g., it finally could not be excluded that the observed narrow structure possibly resulted from detector artifacts. Aside from the question about a light and narrow $\sigma$ meson a problem arises for isoscalar non-fusion two-pion production. In such cases no ABC effect is observed. However, there is no obvious reason, why there the postulated $d^{*}(2380) \rightarrow d \sigma$ decay branch should be suppressed. Kukulin and Platonova argue [206] that due to the much increased phase space for the unbound $N N$ system the centrifugal barrier for the $d$-wave emission of the $\sigma$ meson plays here a much bigger role and might suppress its emission more than in the $d \pi \pi$ case. Unfortunately, a convincing quantitative calculation has not yet been presented by these authors.

Also other small $D$-wave admixtures as they may originate from a $D$-wave admixture in the intermediate $\Delta \Delta$ system [38, 39] or by a hypothetical decay into a $N^{*}(1440) N$ system, as considered to some extent by Bugg [357], may lead to an ABC-effect like behavior as discussed in detail in Ref. [362].

If decays such as $d^{*} \rightarrow D_{12} \pi$ and $d^{*} \rightarrow N^{*}(1440) N$ exist, then there must be also a $d^{*}$ decay branch into the isoscalar $N N \pi$ channel because of the decays $D_{12} \rightarrow N N$ and $N^{*}(1440) \rightarrow N \pi$. The analysis of WASA data on $n p \rightarrow p p \pi^{-}$and $p p \rightarrow p p \pi^{0}$ reactions with the goal to extract the isoscalar single-pion production cross section in the $d^{*}(2380)$ energy region serves the aim to provide an answer for this issue. As mentioned above the preliminary results do not give any indication for such a decay [402].

In conclusion, the $\mathrm{ABC}$ effect turns out to be yet another manifestation that the dominant asymptotic configuration of $d^{*}(2380)$ is a $\Delta \Delta$ system in relative $S$ wave.

\section{Are There More Extraordinary Dibaryons?}

\subsection{Search for the Isospin $I=3$ State $D_{30}$}

Having found one extraordinary dibaryon, of course, raises the question, whether there are more exceptional dibaryon states. In particular, a state with quantum numbers mirrored to those of $d^{*}(2380)$, i.e., with $I\left(J^{P}\right)=3\left(0^{+}\right)$, appears to be particularly appealing. In its highest charge state it would consist 
of just six up quarks. It has been predicted to have a mass similar to that of $d^{*}(2380)$ by a number of theoretical calculations [33, 36, 37, 416], which have been able to predict $d^{*}(2380)$ at the proper mass. The calculations of Mota et al. [205] predict this state to be right at the $\Delta \Delta$ threshold, i.e., much less bound than $d^{*}(2380)$.

Since such a dibaryon is $N N$-decoupled due to its large isospin, it can only be produced in combination with other, associatedly produced particles. For the latter pions are ideally suited due to their large isospin. Hence Dyson and Xuong [33], who were the first to propose such a state (denoted by $D_{30}$, where the first index refers to the isospin and the second one to the spin of this state), suggested to look for this state in the reaction $p p \rightarrow D_{30}^{++++} \pi^{-} \pi^{-} \rightarrow \Delta^{++} \Delta^{++} \rightarrow p p \pi^{+} \pi^{+} \pi^{-} \pi^{-}$. Due to $I=3$ the $\Delta^{++} \Delta^{++}$configuration is the most preferred $\Delta \Delta$ combination, where $D_{30}^{++++}$decays into. For an early attempt to search for an $I=3$ dibaryon state in proton-nucleus collisions see Ref. [417].

Because of the COSY shutdown a dedicated search run could no longer be performed by the WASA collaboration. However, using runs from the WASA data base primarily performed for $\omega$ and $\eta^{\prime}$ production at $\sqrt{s}=2.72$ and $2.88 \mathrm{GeV}$, data sets for exclusive and kinematically complete measurements of four-pion production could be extracted [418]. These constitute the first data in the low-energy regime of this reaction. The obtained $p p \pi \pi$-invariant mass spectra do not show any significant narrow structures and are essentially compatible with a $t$-channel $N^{*}(1440) N^{*}(1440)$ process. By use of detailed peak analyses upper limits for the existence of such an $I=3$ dibaryon in the mass range 2280 - $2500 \mathrm{MeV}$ could be deduced. Of course, above the $\Delta \Delta$ threshold of nominal $2 m_{\Delta}=2464 \mathrm{MeV}$ such a dibaryon state, if existent, would undergo fall-apart decay with an accordingly huge width, which makes any experimental detection practically impossible.

Though no solid evidence for the existence of an $I=3$ dibaryon state below the $\Delta \Delta$ threshold was found, the results are still significant. The currently achievable bounds for $I=3$ dibaryon production are primarily not due to accuracy and statistics of the measurements, but due to the insufficient quantitative knowledge of the conventional mechanisms in four-pion production. So the current limits may be improved, as soon there is progress in the theoretical description of the conventional processes.

Nevertheless, these currently obtained upper limits are more than three orders of magnitude smaller than the formation cross section found for $d^{*}(2380)$. More importantly - with the possible exception of a dibaryon mass of about $2380 \mathrm{MeV}$ combined with a width of about $100 \mathrm{MeV}$ - the upper limits are significantly smaller than the cross section for conventional $\Delta^{++} \Delta^{++}$production in the $p p \pi^{+} \pi^{+} \pi^{-} \pi^{-}$ channel - again in sharp contrast to the corresponding situation for $d^{*}(2380)$ formation, where this is an order of magnitude larger than in conventional $\Delta \Delta$ formation. If the interaction between the two $\Delta^{++}$particles produced side-by-side in the decay of the intermediate $N^{*} N^{*}$ system would be attractive, then the probability to form a dibaryon should be quite large - as it is obviously the case for $d^{*}(2380)$ formation in the presence of an isoscalar $\Delta^{+} \Delta^{0}$ system. However, the WASA results suggest that the probability for dibaryon formation in the presence of a $\Delta^{++} \Delta^{++}$system in the intermediate state is small (with the possible exception of the case mentioned above). This is in support of the findings of Goldman et al. [34, who predicted an attractive interaction between the $\Delta \Delta$ pair in case of $d^{*}(2380)$, but repulsion in case of $D_{30}$ and hence no dibaryon formation.

\subsection{Search for the Isospin $I=2$ State $D_{21}$}

According to Dyson and Xuong [33] and supported by recent Faddeev calculations [36] there should exist a $N N$-decoupled dibaryon state with $I\left(J^{P}\right)=2\left(1^{+}\right)$corresponding asymptotically to a $\Delta N$ system and decaying into $N N \pi$. These calculations predict such a state, called $D_{21}$ by Dyson and Xuong, with a mass slightly below the $\Delta N$ threshold and with a width close to that of the $\Delta$ - featuring thus a state like $D_{12}$ discussed in chapter 4.4 , but with mirrored quantum numbers.

It appears natural to search for such a state in the $p p \rightarrow p p \pi^{+} \pi^{-}$reaction, where such a state would be produced associatedly with an additional pion via the route $p p \rightarrow D_{21}^{+++} \pi^{-} \rightarrow p p \pi^{+} \pi^{-}$. 
Unfortunately there are no high-quality data for this reaction in the energy region of interest. From WASA and COSY-TOF there exist exclusive and kinematically complete measurements from threshold up to $T_{p}=0.8 \mathrm{GeV}(\sqrt{s}=) 2.24 \mathrm{GeV}$. Beyond that there are only poor statistics bubble-chamber measurements. The nominal $\Delta N \pi$ threshold is at $T_{p}=0.96 \mathrm{GeV}(\sqrt{s}=2.31 \mathrm{GeV})$, i.e., for a $D_{21}$ search data above this energy are needed.

In principle there are high-statistics experimental data available from WASA at $T_{p}=1.2 \mathrm{GeV}$ $(\sqrt{s}=2.4 \mathrm{GeV})$ and in particular at $T_{p}=1.4 \mathrm{GeV}(\sqrt{s}=2.48 \mathrm{GeV})$, which originally were intended for $\eta$ production and decay studies. An appropriate analysis of these measurements would allow the search for $D_{21}$ in $p p \pi^{+}$and $p p \pi^{-}$subsystems up to masses of $2.34 \mathrm{GeV}$. Due to isospin coupling $D_{21}$ should show up in the $p p \pi^{+}$subsystem six times stronger than in the $p p \pi^{-}$subsystem, where primarily the reflection of this resonance should be seen.

Though the search procedure would be similar to that used for the $D_{30}$ issue discussed above in the previous section, the search for $D_{21}$ would be under much better conditions. Experimentally there are ten thousands of kinematically overdetermined events expected to be obtainable from the WASA data base. Also with respect to conventional reaction contributions the situation is much superior in this case, since the proton-proton induced two-pion production by $t$-channel meson exchange is understood here on a quantitative level - also with the additional help of isospin relations and isospin decomposition [327]. As shown in Ref. [327] there is a problem with understanding the total cross section beyond $T_{p}$ $=0.9 \mathrm{GeV}(\sqrt{s}=2.29 \mathrm{GeV})$, which happens to coincide with the $\Delta N \pi$ threshold. Beyond this energy the measured total cross sections are too high by up to $30 \%$ compared to the prediction by isospin decomposition. However, all those data originate still from old bubble-chamber measurements.

A hint for the existence of $D_{21}$ comes also from the double-pionic charge exchange reaction on nuclei considered in chapter 5 . There the forward-angle cross section of so-called non-analog transitions exhibits an unexpected resonance-like behavior in the region of the $\Delta$ resonance. For its explanation the so-called DINT (Delta-nucleon INTeraction) mechanism was invented [217, 218, 219, 220], which in essence can be thought of a $\Delta N$ system with $I\left(J^{P}\right)=2\left(1^{+}\right)$in the intermediate state [219] - see chapter 5 .

\subsection{Renaissance of the $\Delta N$ Region: more Resonance Structures}

In chapter 4.4 we discussed the situation in the region of the $\Delta N$ threshold, where clear resonance structures with the width of the $\Delta$ resonance were observed in the past. Especially pronounced appear these structures in the $p p \rightarrow d \pi^{+}$reaction. From a combined SAID partial-wave analysis of this reaction with $p p$ elastic scattering a looping in the Argand diagram has been observed for the ${ }^{1} D_{2},{ }^{3} P_{2^{-}}{ }^{3} F_{2},{ }^{3} F_{3}$ and ${ }^{3} F_{4^{-}}{ }^{3} H_{4} p p$-partial waves corresponding to isovector states with $J^{P}=2^{+}, 2^{-}, 3^{-}$and $4^{-}$. The first one represents the by far most pronounced resonance structure. It corresponds asymptotically to a slightly bound $\Delta N$ system in relative $S$ wave, whereas in the other cases this system has to be in relative $P$ wave.

In a very recent series of measurements carried out at the ANKE detector at COSY the $\overrightarrow{p p} \rightarrow p p \pi^{0}$ reaction has been investigated under the condition that the emitted proton pair is in relative $S$-wave, i.e. in the ${ }^{1} S_{0}$ state [419]. That way the ANKE measurements are complementary to the reaction $p p \rightarrow d \pi^{+}$, where the nucleons within the deuteron are in relative ${ }^{3} S_{1}$ state - neglecting the small $D$-wave admixture in the deuteron.

In a very careful phenomenological analysis of their data as well as of corresponding data from PROMICE/WASA [420] over the energy range from close-to-threshold up to $T_{p}=1.1 \mathrm{GeV}(\sqrt{s}=2.36$ $\mathrm{GeV})$ the ANKE collaboration finds the transitions ${ }^{3} P_{0} \rightarrow{ }^{1} S_{0} s$ and ${ }^{3} P_{2} \rightarrow{ }^{1} S_{0} d$ to exhibit pronounced Lorentzian-shaped resonance structures. Fitted by an Breit-Wigner ansatz these structures correspond to $I\left(J^{P}\right)=1\left(0^{-}\right)$and $1\left(2^{-}\right)$resonances with masses $2201 \pm 5 \mathrm{MeV}$ and $2197 \pm 8 \mathrm{MeV}$, respectively, and with widths of $91 \pm 12 \mathrm{MeV}$ and $130 \pm 21 \mathrm{MeV}$, respectively. These values for mass and width suggest 


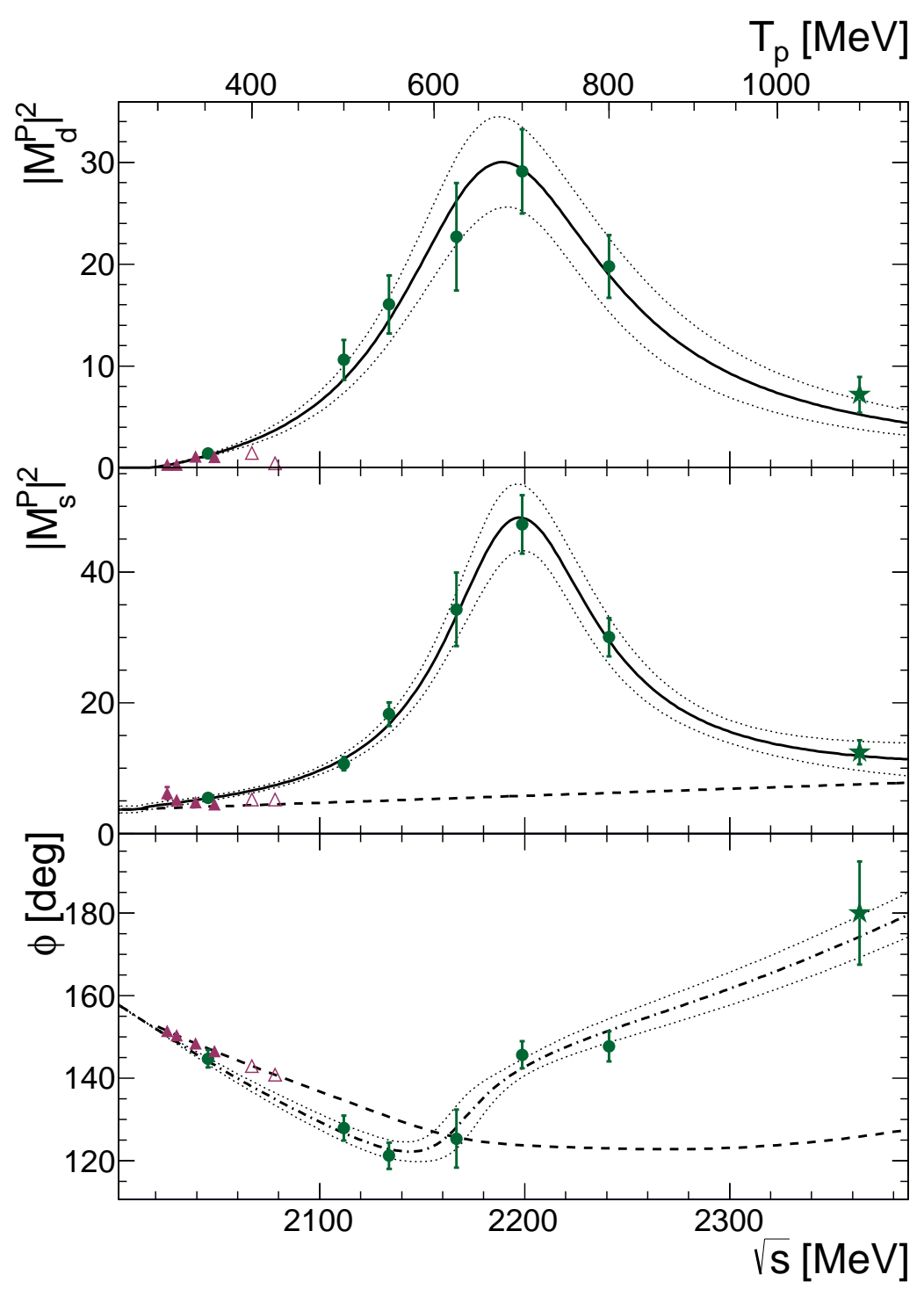

Figure 15: Energy dependence of the transition amplitudes squared for the transitions ${ }^{3} P_{2} \rightarrow^{1} S_{0} d$ (top) and ${ }^{3} P_{0} \rightarrow{ }^{1} S_{0} s$ (middle) as well as their relative phase (bottom). Filled circles and star denote ANKE measurements [419], whereas triangles give PROMICE/WASA results [420]. The solid lines represent Breit-Wigner resonance fits with an empirical ansatz (dash-dotted line) for the relative phase, the corridors indicated by the dashed lines give the $68 \%$ confidence interval. The dotted line in the middle panel shows the assumed background and the dotted line in the bottom panel the relative phase of the ${ }^{3} P_{0}$ and ${ }^{3} P_{2}$ amplitudes in elastic $p p$ scattering. From [419]. 
the resonance structures to represent $\Delta N$ configurations, where due to spin and parity $\Delta$ and $N$ have to be in relative $P$ wave.

The $I\left(J^{P}\right)=1\left(2^{-}\right)$resonance structure has been observed already before in SAID partial-wave analyses. The ANKE results for mass and width of this resonance structure are in reasonable agreement with the pole position of $(2167-i 86) \mathrm{MeV}$ obtained in the SAID analysis of $p p$ scattering [190]. According to Ref. [419] the pole position obtained in the more recent (unpublished) SAID solution SP96 for the $p p \rightarrow d \pi^{+}$reaction is $(2192-i 64) \mathrm{MeV}$, which is even in perfect agreement with the ANKE result.

Recently Kukulin and Platonova have demonstrated that the spin correlation parameters are particularly sensitive to this resonance [207]. That way they are able to describe all measured cross section and polarisation data of the $p p \rightarrow d \pi^{+}$reaction for the first time in a comprehensive and quantitative manner.

The finding of a $I\left(J^{P}\right)=1\left(0^{-}\right)$state is new, the corresponding transition ${ }^{3} P_{0} \rightarrow{ }^{1} S_{0} s$ is forbidden in the $p p \rightarrow d \pi^{+}$reaction. Whereas the width of the $I\left(J^{P}\right)=1\left(0^{-}\right)$state appears to be slightly less than the width of the $\Delta$ isobar, the width of the $I\left(J^{P}\right)=1\left(2^{-}\right)$tends to be slightly larger.

The masses of both resonance structures coincide within their uncertainties and are about $30 \mathrm{MeV}$ above the nominal $\Delta N$ mass. In ref. [419] it is argued that the orbital $P$-wave motion should lead to an increase of the effective mass by roughly $60 \mathrm{MeV}$. Relative to that the observed masses appear to signal a binding of about $30 \mathrm{MeV}$ indicating thus an attractive $P$-wave $\Delta N$ interaction.

At the CLAS spectrometer at JLAB the $\gamma d \rightarrow d \pi^{+} \pi^{-}$reaction has been investigated recently. Preliminary results have been reported at the APS Spring meeting 2014 and at BARYON 2016. No sign of $d^{*}$ (2380) has been observed as actually expected - see chapter 10.5.1 -, since this reaction channel is overwhelmed by conventional processes. However, the Dalitz plot of the $d \pi^{+}$-invariant mass-squared versus the $d \pi^{-}$-invariant mass-squared exhibits a pronounced broad resonance line symmetric in both invariant mass systems slightly below the nominal $\Delta N$ threshold. Spin and parity of this isovector structure have not yet been reported.

\subsection{Upcoming Results for the $\Omega^{-} p$ system $(S=-3)$}

Since in heavy ion collisions hyperons are produced copiously, this situation can be used to investigate the hyperon-hyperon interaction by measuring hyperon-hyperon correlations. Such correlations of Hanbury-Brown-Twiss [21] type are usually used to fix the size of the source, which emits the particular particles. But if the source size is known, e.g. from the study of other channels, such correlations can be used to deduce scattering length and effective range of particle-particle interactions [422]. After the deduction of the $\Lambda \Lambda$ scattering length in search of the $H$ dibaryon- see section 7 - the STAR collaboration has now also measured the $\Omega^{-} p$ correlation. The result is expected to be released in nearest future.

From the theoretical side a $\Omega^{-} p$ bound state has been predicted by several groups. Goldman et al. found by use of two different quark models that there should be two bound states with $J=1$ and 2 being stable against strong decay [96]. Oka found out by use of a constituent quark model that there should be a quasi-bound state with $I\left(J^{P}\right)=\frac{1}{2}\left(2^{+}\right)$[93]. Also Li and Shen observe this state to be bound within their chiral quark model approach [423. And very recently lattice QCD calculations by the HALQCD collaboration report such a state being bound by $19 \mathrm{MeV}$ - though the pion mass of 875 $\mathrm{MeV}$ used in these calculations is still far off the physical value [424].

\subsection{Are there Dibaryons in the Heavy-Quark Sector?}

The most convincing evidence for the existence of tetraquark and pentaquark systems comes from experiments performed in sectors, where charmed and bottom quarks are involved. This suggests that 
the attraction between quark clusters is largest, if each of the two clusters contain a heavy quark (or anti-quark). This has been worked out recently also for the dibaryon scenario by Karliner et al. [425, 426, 427, 428] predicting, e.g., a doubly heavy $\Sigma_{b}^{+} \Sigma_{b}^{-}$dibaryon.

Pion assisted dibaryon candidates with charm have been discussed recently by Gal et al. [9, 429] within the chiral constituent quark model [430]. In this work the same formalism as applied earlier to the $\Lambda N \pi$ system [278] with strangeness $S=-1$ was applied to the $\Lambda_{c} N \pi$ system with charm $C=1$. That way $\Lambda(1116)$ and $\Sigma(1385)$ have been replaced by the charmed analogs $\Lambda_{c}(2286)$ and $\Sigma_{c}(2520)$. Faddeev equations with relativistic kinematics were solved to look for bound states and resonances with $I\left(J^{P}\right)=\frac{3}{2}\left(2^{+}\right)$. Depending on the tested model parameters a bound state or resonance was generated, which may be viewed as $\Sigma_{c}(2520) N$ bound state. It could likely be the lowest lying charmed dibaryon - below the mass of about $3500 \mathrm{MeV}$ predicted recently in Ref. [431] for a $D N N$ bound state.

The $D N N$ bound state would be analogous to the $\bar{K} N N$ system discussed in section 6 . by substituting the $\bar{K}$ by a $D$ meson. Due to the attractive interaction in the isoscalar part of the $D N$ interaction a $D N N$ system with $I\left(J^{P}\right)=\frac{1}{2}\left(0^{-}\right)$is expected to be bound by about $250 \mathrm{MeV}$ relative to the $D N N$ threshold with a narrow width of only $20-40 \mathrm{MeV}$. Hence it should be observable much easier than the $\bar{K} N N$ state and may be interpreted as a quasibound $\Lambda_{c}(2595) N$ system [431].

There are not yet any experimental results for this issue. Possible searches on this issue should be feasible at J-PARC, RHIC, LHC. Also, if PANDA at FAIR should be realized in some foreseeable future, it would be a top installation for dibaryon searches in the heavy-quark sector.

\section{Conclusions and Outlook}

The history of dibaryons has been marked by a period of enthusiasm, the dibaryon rush era, followed by a period of great frustration, where the buzzword "dibaryon" caused immediate aversions in audiences and committees. The reason was that in the nineties an overwhelming number of dibaryon claims turned out not to survive a careful experimental examination.

The only remaining experimentally established resonance-like structures from this period have been the narrow structure at the $\Sigma N$ threshold and the broad structures near the $\Delta N$ threshold with widths close to that of the $\Delta$ resonance. The narrow structure at the $\Sigma N$ threshold has been reexamined by exclusive and kinematically complete measurements of the $p p \rightarrow \Lambda p K^{+}$reaction. It agrees with the cusp effect due to the coupling of $\Lambda p$ and $\Sigma N$ channels. The broad $\Delta N$ resonance structures have been further established by observation in various channels. Whether they constitute genuine $s$-channel resonances is still not definitely settled. Anyway they resemble loosely bound $\Delta N$ systems - presumably without significant exotic quark configurations.

So we are left so far with a single firmly established non-trivial dibaryon state, the one with $I\left(J^{P}\right)=$ $0\left(3^{+}\right)$at $2380 \mathrm{MeV}$. Its dynamic decay properties point to an asymptotic $\Delta \Delta$ configuration, bound by $80-90 \mathrm{MeV}$. Its width of only $70 \mathrm{MeV}$ - being more than three times smaller than expected for a conventional $\Delta \Delta$ system excited by $t$-channel meson exchange - points to an exotic origin like hidden-color effects.

Though the observation of such a state came for many as a surprise, it was predicted properly already as early as 1964 by Dyson and Xuong. After having established experimentally the first nontrivial dibaryon state the agreement with the early prediction by Dyson and Xuong appears to be very remarkable. All four states predicted to be coupled to the $N N$ system have now been observed - with the first three being the "trivial" states deuteron, virtual ${ }^{1} S_{0}$ state and ${ }^{1} D_{2} N N$ scattering state at the $\Delta N$ threshold. The remaining two states of the predicted sextet are $N N$-decoupled having $\mathrm{I}=2$ and 3 , respectively, but may be searched for in $N N$-initiated multi-pion production. Though the hadron physics program has finished at COSY, there is still a wealth of WASA data available, which are suited to search also for these states. 
It comes a bit as a surprise that not a single dibaryonic state has yet been established in the strange sector - despite of many dedicated searches. This includes also the $H$ dibaryon, which once initiated the dibaryon rush. Though many sophisticated and state-of-the-art experimental searches have been conducted in recent times, no sign of its existence has been found - be it as bound or resonant object. From hyperon-nucleon studies we know that the hyperon-nucleon interaction is substantially weaker than the nucleon-nucleon interaction. From this we understand that there is no bound strange deuteron. Obviously it is also too weak to allow for resonances. Whether the current hints for a loosely bound molecular-like $p p K^{-}$system will materialize has to be seen by future experiments. From RHIC experiments we see that the hyperon-hyperon interaction tends to be still weaker. However, there is hope that these kind of heavy-ion experiments may give us an answer, whether in the $S=-3$ system the $\Omega^{-} p$ configuration has a bound state.

The fact that tetra- and penta-quark systems have been observed not in the strange, but in heavyquark sector, gives confidence that the baryon-baryon interaction there is more attractive than in the strange sector. This is also borne out by a number of model calculations, which support the hope that in this sector also dibaryonic hexaquark systems will be found in near future.

\section{Acknowledgments}

The work on this issue would not have been possible without intense communications and discussions lasting partly over decades, for which I am particularly grateful to Dick Arndt, W. Briscoe, Stanley J. Brodsky, D. Bugg, Y. B. Dong, E. Friedman, A. Gal, T. Goldman, W. Gibbs, Ch. Hanhart, M. B. Johnson, V. Kukulin, E. Oset, M. Platonova, P. N. Shen, M. Schepkin, K. K. Seth, I. Strakovsky, A. Valcarce, F. Wang, C. Wilkin, R. Workman and Z. Y. Zhang. I am particularly indebted to my Tübingen colleagues M. Bashkanov, A. Buchmann, A. Faessler, Th. Gutsche, H. Müther, T. Skorodko and G. J. Wagner - and all my former students - for their enduring help in all kinds of problems. The finally successful dibaryon search would not have been possible without the intense support by the WASA collaboration and the staffs of CELSIUS and COSY.

I am grateful to all of those, who were very sceptical about the dibaryon issue and thus forced us to go through all the cumbersome tests of the dibaryon hypothesis.

This work has been supported by DFG(CL 214/3-1 and 3-2) and in the years before by BMBF and Forschungszentrum Jülich (COSY-FFE).

\section{References}

[1] Harold C. Urey, F. G. Brickwedde, and G. M. Murphy, Phys. Rev. 39, 164 (1932).

[2] A. Yokosawa, Phys. Rep. 64 (1980) 47

[3] M. P. Locher, M. E. Sainio and A. Svarc, Adv. Nucl. Phys. 17 (1986) 47

[4] I. I. Strakovsky, Sov. J. Part. Nucl. 22 (1991) 296

[5] K. K. Seth, Proc. Baryon-Baryon Interaction and Dibaryonic Systems, Bad Honnef 1988, (1988) 41

[6] K. K. Seth, Proc. Int. Conf. on Medium and High-Energy nuclear Physics, Taipei, 1988, edited by W.-Y. Pauchy Hwang, K.-F. Liu and Y. Tzeng (World Scientific, Singapore, 1989).

[7] B. Tatischeff, M. P. Comets, Y. Le Bornec, N. Willis, M. Bolvin, J. Yonnet, preprint IPNO-DRE 91-18 (1991) and IPNO-DRE 92-16 (1992) 
[8] E. N. Komarov, Russian Academy of Sciences, Petersburg Nuclear Physics Institute, preprint 1853 (1993)

[9] A. Gal, Acta Phys. Polon. B 47 (2016) 471

[10] A. Gal, Proc. MESON 2016, EPJ Web Conf. 130 (2016) 01030

[11] M. Bashkanov, Stanley J. Brodsky and H. Clement, Phys. Lett. B 727 (2013) 438

[12] S. R. Beane et al., Phys. Rev. Lett. 106 (2011) 162001

[13] S. R. Beane et al., Phys. Rev. D 87 (2013) 034506

[14] T. Inoue et al., Phys. Rev. Lett. 106 (2011) 162002

[15] T. Inoue et al., arxiv: 1212.4230 [hep-lat]

[16] R. L. Jaffe, Phys. Rev. Lett. 138 (1977) 195 and 617(E)

[17] H. Takahashi et al., Phys. Rev. Lett. 87 (2001) 212502

[18] K. Nakazawa et al., Nucl. Phys. A 835 (2010) 207

[19] J. K. Ahn et al., Phys. Lett. B 444 (1998) 267

[20] C. J. Yoon et al., Phys. Rev. C 75 (2007) 022201(R)

[21] B. H. Kim et al., Phys. Rev. Lett. 110 (2013) 222002

[22] J. Adam et al., Phys. Lett. B 752 (2016) 267

[23] L. Adamczyk et al., Phys. Rev. Lett. 114 (2015) 022301

[24] K. Morita, T. Furumoto and A. Ohnishi, Phys. Rev. C 91 (2015) 024916

[25] A. Ohnishi, K. Morita, K. Miyahara and T. Hyodo, Nucl. Phys. A 954 (2016) 294

[26] P. Adlarson et al., Phys. Rev. Lett. 106 (2011) 242302

[27] M. Bashkanov et al., Phys. Rev. Lett. 102 (2009) 052301

[28] P. Adlarson et al., Phys. Lett. B 721 (2013) 229

[29] P. Adlarson et al., Phys. Rev. C 88 (2013) 055208

[30] P. Adlarson et al., Phys. Lett. B 743(2015) 325

[31] P. Adlarson et al., Phys. Rev. Lett. 112 (2014) 202301

[32] P. Adlarson et al., Phys. Rev. C 90 (2014) 035204

[33] F. J. Dyson and N.-H. Xuong, Phys. Rev. Lett. 13 (1964) 815 and references therein.

[34] T. Goldman, K. Maltman, G. J. Stephenson, K. E. Schmidt and Fan Wang, Phys. Rev. C 39 (1989) 1889

[35] A. Gal and H. Garcilazo, Phys. Rev. Lett. 111 (2013) 172301 
[36] A. Gal and H. Garcilazo, Nucl. Phys. A 928 (2014) 73

[37] H. Huang,J. Ping and F. Wang, Phys. Rev. C 89 (2014) 034001

[38] X. Q. Yuan, Z. Y. Zhang, Y. W. Yu and P. N. Shen, Phys. Rev. C 60 (1999) 045203

[39] F. Huang, Z. Y. Zhang, P. N. Shen and W. L. Wang, Chin. Phys. C 39 (2015) 071001

[40] K. A. Olive et al. (Particle Data Group), Chin. Phys. C 38 (2014) 090001

[41] H. de Vries, C. W. de Jager and C. de Vries, Atomic Data and Nuclear Data Tables 36 (1987) 495

[42] CODATA recommended values.

[43] L. Hulthen and M. Sugawara: The two-nucleon problem, in S. Flügge, Handbuch der Physik 39 (1957)

[44] R. V. Reid JR., Ann. Phys. 50 (1968) 411

[45] see e.g.: H. Dijk and B. L. G. Bakker, Nucl. Phys. A 494 (1989) 438

[46] G. A. Miller, Phys. Rev. C 89 (12014) 045203

[47] B. Julia-Diaz, D. R. Entem, A. Valcarce and F. Fernandez, Phys. Rev. C 66 (2002) 047002

[48] V. I. Kukulin, I. T. Obukhovsky, V. N. Pomerantsev, and A. Faessler, Int. J. Mod. Phys. E 11 (2002) 1

[49] D. Allasia et al., Phys. Lett. B 174 (1986) 450

[50] R. Dymarz and F. C. Khanna, Nucl. Phys. A 516 (1990) 549

[51] A. N. Ivanov, H. Oberhummer, N. I. Troitskaya and M. Faber, Eur. Phys. J. A 8 (2000) 125

[52] R. Machleidt, Phys. Rev. C 63 (2001) 024001

[53] O. Schori et al., Phys. Rev. C 35 (1983) 2252

[54] B. Gabioud et al., Nucl. Phys. A 420 (1984) 496

[55] H. P. Noyes, Ann. Rev. Nucl. Part. Sci. 22 (72) 465

[56] V. Huhn et al., Phys. Rev. C 63 (2000) 024001

[57] V. V. Flambaum and R. B. Wiringa, Phys. Rev. C 76 (2007) 054002

[58] A. B. Migdal, JETP 28 (1955) 1

[59] K. W. Watson, Phys. Rev. 88 (1952) 1163

[60] F. Hauenstein et al., arXiv:1607.04783 [nucl-ex]

[61] G. Alexander et al., Phys. Rev. 173 (1968) 1452

[62] A. Budzanowski et al., Phys. Lett. B 687 (2010) 31

[63] J. Haidenbauer and U.-G. Meissner, Phys. Rev. C 72 (2005) 044005 
[64] T. Rijken, V. Stoks and Y. Yamamoto, Phys. Rev. C 59 (1999) 21

[65] J. Haidenbauer et al., Nucl. Phys. A 915 (2013) 24

[66] M. Abdel-Bary et al., Eur. Phys. J. A 48 (2012) 23

[67] M. Abdel-Bary et al., Eur. Phys. J. A 46 (2010) 27

[68] B. S. Neganov and L. B. Parfenov, Soviet Phys.-JETP 7 (1958) 528

[69] B. S. Neganov and O. V. Savchenko, Soviet Phys.-JETP 5 (1957) 1033

[70] M. G. Meshcheriakov and B. S. Neganov, Dokl. Akad. Nauk SSSR 100 (1955) 677

[71] M. Gell-Mann, Phys. Lett. B 8 (1964) 214

[72] R. Oakes, Phys. Rev. 131 (1963) 2239

[73] M. Gell-Mann, Phys. Rev. 125 (1962) 1067

[74] S. Okubo, Prog. Theor. Phys. 27 (1962) 949

[75] P. J. G. Mulders, A. Th. M. Aerts and J. J. de Swart, Phys. Rev. Lett. 40 (1978) 1543

[76] A. Th. M. Aerts, P. J. G. Mulders and J. J. de Swart, Phys. Rev. D 17 (1978) 260

[77] P. J. Mulders, A. T. Aerts and J. J. de Swart, Phys. Rev. D 21 (1980) 2653

[78] P. J. Mulders, Phys. Rev. D 26 (1982) 3039

[79] P. J. Mulders and Anthony W Thomas, J. Phys. G 9 (1983) 1159

[80] Koichi Saito, Prog. Theor. Phys. 72 (1984) 674

[81] R. P. Bickerstaff, Phil. Trans. R. Soc. Lond. A 309 (1983) 611

[82] A. T. M. Aerts and C. B. Dover, Phys. Rev. D 28 (1983) 450

[83] A. T. M. Aerts and C. B. Dover, Phys. Lett. B 146 (1984) 95

[84] A. T. M. Aerts and C. B. Dover, Nucl. Phys. B 253 (1985) 116

[85] P. LaFrance and E. L. Lomon, Phys. Rev. D 34 (1986) 1341

[86] P. Gonzales, P. LaFrance and E. L. Lomon, Phys. Rev. D 35 (1987) 2142

[87] E. L. Lomon, Few-Body Systems, Suppl. $7 /(1994) 213$

[88] L. A. Kondratyuk. B. V. Martem'yanov and M. G. Shchepkin, Sov. J. Nucl. Phys. 45 (1987) 776

[89] B. V. Martem'yanov and M. G. Shchepkin, JETP Lett. 53 (1991) 139

[90] I. Yu. Kobzarev. B. V. Martem'yanov and M. G. Shchepkin, Sov. Phys. Usp. 35 (1992) 257

[91] M. Oka and K. Yazaki, Phys. Lett. B 90 (1980) 41

[92] M. Oka, K. Shimizu and K. Yazaki, Phys. Lett. B 130 (1983) 365 
[93] M. Oka, Phys. Rev. D 38 (1988) 298

[94] Kim Maltman, Nucl. Phys. A438 (1985) 669

[95] Kim Maltman, Nucl. Phys. A 501 (1989) 843

[96] T. Goldman, K. Maltman, G. J. Stephenson, K. E. Schmidt and Fan Wang, Phys. Rev. Lett. 59 (1987) 627

[97] T. Goldman, Nucl. Phys. A 532 (1991) 389c

[98] T. Barnes, S. Capstick, M. D. Kovarik and E.S. Swanson, Phys. Rev. C 48 (1993) 539

[99] T. Kamae and T. Fujita, Phys. Rev. Lett. 38 (1977) 471

[100] H. Sato and K. Saito, Phys. Rev. Lett. 50 (1983) 648

[101] Humberto Garcilazo, Phys. Rev. C 26 (1982) 2685

[102] H. Garcilazo and L. Mathelitsch, Phys. Rev. C 34 (1986) 1425

[103] W. A. Gale and I. M. Duck, Nucl. Phys. B 8 (1968) 109

[104] G. Kalbermann and J. M. Eisenberg, J. Phys. G 5 (1977) 35

[105] T. Ueda, Phys. Lett. B 74 (1978) 123

[106] M. Dillig, see citation in Ref.[158].

[107] Shu-Qin Xie, J. Phys. G 15 (1989) 287

[108] C. Lechanoine-LeLuc and F. Lehar, Rev. Mod. Phys. 65 [1993) 47

[109] V. G. J. Stoks, R.A.M. Klomp, C.P.F. Terheggen, and J.J. de Swart, Phys. Rev. C 49 (1993) 2950

[110] R. A. Arndt, I. I. Strakovsky and R. L. Workman, Phys. Rev. C 50 (1994) 2731

[111] R. A. Arndt, I. I. Strakovsky and R. L. Workman, Phys. Rev. D 50 (1994) 1796

[112] I. P. Auer et al., Phys. Rev. Lett. 62 (1989) 2649

[113] J. Ball et al., Phys. Lett. B 320 (1994) 206

[114] R. A. Arndt, I. I. Strakovsky, R. L. Workman and D. V. Bugg, Phys. Rev. C 48 (1993) 1926

[115] Y. A. Troyan and V. N. Pechenov, Phys. At. Nucl. 56 (1993) 528

[116] B. M. Abramov et al., Z. Phys. C 69 (1996) 409

[117] Yu. A. Troyan, V. N. Pechenov, E. B. Plekhanov, A. Yu. Troyan, S. G. Arakelyan, V. I. Moroz and A. P. Jerusalimov, Phys. At. Nucl. 63 (2000) 1562

[118] B. Tatischeff et al., Phys. Rev. C 59 (1999) 1878

[119] B. Tatischeff et al., Phys. Rev. C 45 (1992) 2005

[120] V. A. Krasnov et al., Phys. Lett. B 108 (1982) 11 
[121] J. Julien et al., Phys. Lett. B 142 (1984) 340

[122] V. Aseev et al., Phys. Rev. C 56 (1997) R596

[123] S. Yen et al., Phys. Lett. B 269 (1991) 59

[124] J. Julien et al., Z. Phys. A 347 (1994) 181

[125] A. B. Kurepin andK. O. Oganesian, JETP Lett. 49 (1989) 694

[126] E. A. Pasyuk et al., Phys. Rev. C 55 (1997) 1026

[127] F. F. Liu, D. E. Lundquist and B. H. Wiik, Phys. Rev. 165 (1968) 1478

[128] R. Kose et al., Z. Phys. 220 (1969) 305

[129] T. Kamae et al., Phys. Rev. Lett. 38 (1977) 468

[130] T. Kamae et al., Nucl. Phys. B 139 (1978) 394

[131] H. Ikeda et al., Phys. Rev. Lett. 42 (1979) 1321

[132] H. Ikeda et al., Nucl. Phys. B 172 (1980) 509

[133] P. E. Argan et al., Phys. Rev. Lett. 46 (1981) 96

[134] W. Ruhm et al., Nucl. Phys. A 459 (1986) 557

[135] G. Tamas, Nucl. Phys. A 358 (1981) 347c

[136] J. Arvieux et al., Nucl. Phys. A 444 (1985) 579

[137] B. Bock et al., Nucl. Phys. A 459 (1986) 573

[138] Y. Ilieva et al., Eur. Phys. J. A 43 (2010) 261

[139] U. Siodlaczek et al., Eur. Phys. J. A 9 (2000) 309

[140] L. V. Fil'kov, Sov. J. Nucl. Phys. 47 (1988) 437

[141] D. M. Akhmedov and L. V. Fil'kov, Nucl. Phys. A 544 (1992) 692

[142] L. V. Fil'kov et al., Phys. Rev. C 61 (2000) 044004

[143] L. V. Fil'kov et al., Eur. Phys. J. A 12 (2001) 369

[144] A. Cichocki, PhD thesis, Univ. Virginia (2003)

[145] A. Tami et al., Phys. Rev. C 65 (2002) 047001

[146] A. Tami et al., Nucl. Phys. A 721 (2003) 621c

[147] S. B. Gerasimov and A. S. Khrykin, Mod. Phys. Lett. A 26 (1993) 2457

[148] A. S. Khrykin et al., in: Proc. VI Int. Symp. on Meson-Nucleon Physics and the Struture of the Nucleon, Blaubeuren/Tübingen, 1995, $\pi N$ Newsletter 10 (1995) 67 
[149] A. S. Khrykin et al., in: Proc. VII Int. Symp. on Meson-Nucleon Physics and the Struture of the Nucleon, Vancouver, 1997, $\pi N$ Newsletter 13 (1997) 67

[150] A. S. Khrykin et al., Phys. Rev. C 64 (2001) 034002

[151] A. S. Khrykin et al., Nucl. Phys. A721 (2003) 625c

[152] H. Calen et al., Phys. Lett. B427 (1998) 248

[153] E. Paisetzky et al., Phys. Rev. Lett. 53 (1984) 540

[154] F. W. N. de Boer et al., Phys. Rev. Lett. 53 (1984) 423

[155] J. Lichtenstadt et al., Phys. Rev. C33 (1986) 665

[156] D. Ashery et al., Phys. Lett. B 215 (1988) 41

[157] E. Paisetzky et al., Phys. Lett. B 219 (1989) 237

[158] B. Parker et al., Phys. Rev. Lett. 63 (1989) 1570

[159] Y. I. Azimov and I. I. Strakovsky, Sov. J. Nucl. Phys. 51 (1990) 384

[160] O. I. Dahl et al., Phys. Rev. Lett. 6 (1961) 142

[161] D. Cline, R. Laumann and J. Mapp, Phys. Phys. Rev. Lett. 20 (1968) 1452

[162] G. Alexander et al., Phys. Rev. 173 (1968) 1452

[163] T. H. Tan, Phys. Rev. Lett. 23 (1969) 395

[164] W. H. Sims et al., Phys. Rev. D 3 (1971) 1162

[165] D. Eastwood et al., Phys. Rev. D 3 (1971) 2603

[166] O. Braun et al., Nucl. Phys. B 124 (1977) 45

[167] C. Pigot et al., Nucl. Phys. B 249 (1985) 172

[168] S. M. Flatté, Phys. Lett. B 63 (1976) 224

[169] G. Toker, A. Gal and J. M. Eisenberg, Nucl. Phys. A 362 (1981) 405

[170] M. Torres, R. H. Dalitz and A. Deloff, Phys. Lett. B 174 (1986) 213

[171] A. M. Badalyan, L. P. Kok, M. I. Polikarpov and Y. A. Simonov, Phys. Rep. 82 (1982) 31

[172] A. C. Melissinos et al., Phys. Rev. Lett. 14 (1965) 604

[173] J. T. Reed et al., Phys. Rev. 168 (1968) 1495

[174] W. J. Hogan, P.A. Piroué and A. J. S. Smith, Phys. Rev. 166 (1968) 1472

[175] R. Siebert et al., Nucl. Phys. A567 (1994) 819

[176] A. Deloff, Nucl. Phys. A 505 (1989) 583

[177] J. M. Laget, Phys. Lett. B 259 (1991) 24 
[178] A. Budzanowski et al., Phys. Rev. D 84 (2011) 032002

[179] A. Budzanowski et al., Phys. Lett. B 692 (2010) 10

[180] H. Machner et al., Nucl. Phys. A 901 (2013) 65

[181] R. A. Arndt, Phys. Rev. 165 (1968) 1834

[182] A. V. Kravtsov, M. G. Ryskin and I. I. Strakovsky, J. Phys. G 9 (1983) L 187

[183] I. I. Strakovsky, A. V. Kravtsov and M. G. Ryskin, Sov. J. Nucl. Phys. 40 (1984) 273

[184] M. J. Borkowski et al., J. Phys. G 11 (1985) 69

[185] R. Bhandari, R. A. Arndt, L. D. Roper and B. J. VerWest, Phys. Rev. Lett. 46 (1981) 1111

[186] R. A. Arndt, I. Strakovsky, R. L. Workman and D. V. Bugg, Phys. Rev. C 48 (1993) 1926

[187] C. H. Oh, R. A. Arndt, I. Strakovsky and R. L. Workman, Phys. Rev. C 56 (1997) 635

[188] R. A. Arndt, L. D. Roper, R. A. Bryan, R. B. Clark, B. J. VerWest and P. Signell, Phys. Rev. D 28 (1983) 97

[189] R. A. Arndt, J. S. Hyslop III and L. D. Roper, Phys. Rev. D 35 (1987) 128

[190] R. A. Arndt, L. D. Roper, R. L. Workman and M. W. McNaughton, Phys. Rev. C 45 (1992) 3995

[191] D. D. Brayshaw, Phys. Rev. Lett. 37 (1976) 1329

[192] B. L. G. Bakker, I. M. Narodetsky and Y. A. Simonov, Lett. Nouvo Cim. 19 (1977) 265

[193] I. M. Narodetsky and Y. A. Simonov, Sov. J. Nucl. Phys. 28 (1978) 698

[194] Y. A. Simonov and M. van der Velde, Phys. Lett. B 76 (1978) 277

[195] Y. A. Simonov, Sov. Phys. Usp 25 (1982) 99

[196] J. A. Niskanen, Phys. Lett. B 112 (1982) 17

[197] R. L. Shypit et al., Phys. Rev. Lett. 60 (1988) 901

[198] R. L. Shypit et al., Phys. Rev. C 40 (1989) 2203

[199] V. V. Anisovich, A. V. Sarantsev and D. V. Bugg, Nucl. Phys. A 537 (1992) 501

[200] M. G. Ryskin and I. I. Strakovsky, Phys. Rev. Lett. 61 (1988) 2384

[201] N. Hoshizaki, Phys. Rev. C 45 (1992) R1414

[202] N. Hoshizaki, Prog. Theor. Phys. 89 (1993) 245, ibid. 251, 563, 569

[203] T. Ueda, Y. Ikegami, K. Tada and K. Kameyama, Few Body Systems Suppl. 9 (1995) 177

[204] R. D. Mota, A. Valcarce, F. Fernández and H. Garcilazo, Phys. Rev. C 59 (1999) 46

[205] R. D. Mota, A. Valcarce, F. Fernández, D. R. Entem and H. Garcilazo, Phys. Rev. C 65 (2002) 034006 
[206] M. N. Platonova and V. I. Kukulin, Nucl. Phys. A 946 (2016) 117

[207] M. N. Platonova and V. I. Kukulin, Phys. Rev. D 94 (2016) 054039

[208] H. Clement, Prog. Part. Nucl. Phys. 29 (1992) 175

[209] M. B. Johnson and C. L. Morris, Ann. Rev. Part. Sci. 43 (1993) 165

[210] S. J. Greene, C. J. Harvey; P. A. Seidl, R. Gilman; E. R. Siciliano and M. B. Johnson, Phys. Rev. C 30 (1984) 2003

[211] Gerald A. Miller, Phys. Rev. Lett. 53 (1984) 2008

[212] M. J. Leitch, in: "Proc. Second LAMPF Workshop on Pion-Nucleus Double Charge Exchange", Los Alamos, New Mexico, USA, 1989 (W. R. Gibbs and M. J. Leitch, eds.), World Scientific (1990) 27

[213] M. J. Leitch et al., Phys. Rev. C 39 (1989) 2356

[214] Gerald A. Miller, Phys. Rev. C 35 (1987) 377

[215] G. R. Burleson, in: "Proc. Second LAMPF Workshop on Pion-Nucleus Double Charge Exchange", Los Alamos, New Mexico, USA, 1989 (W. R. Gibbs and M. J. Leitch, eds.), World Scientific (1990) 79

[216] R. Gilman et al., Phys. Rev. C 35 (1987) 1334

[217] Mikkel B. Johnson, E. R. Siciliano, H. Toki and A. Wirzba, Phys. Rev. Lett. 52 (1984) 593

[218] R. Gilman, H. T. Fortune, M. B. Johnson, E. R. Siciliano, H. Toki and A. Wirzba, Phys. Rev. C $32(1985) 349$

[219] Mikkel B. Johnson and L. S. Kisslinger, Phys. Lett. B 168 (1986) 26

[220] A. Wirzba, H. Toki, E. R. Siciliano, M. B. Johnson and R. Gilman, Phys. Rev. C 40 (1989) 2745

[221] J. A. Faucett et al., Phys. Rev. C 35 (1987) 1570

[222] R. Bilger et al., Phys. Lett. B 269 (1991) 247

[223] R. Bilger et al., Z. Phys. A 343 (1992) 491

[224] K. Föhl et al., Phys. Rev. Lett. 79 (1997) 3849

[225] J. Pätzold et al., Phys. Lett. B 428 (1998) 18

[226] J. Pätzold et al., Phys. Lett. B 443 (1998) 77

[227] J. Draeger et al., Phys. Rev. C 62 (2000) 064615

[228] R. Bilger, H. A. Clement and M. G. Schepkin, Phys. Rev. Lett. 71 (1993) 42

[229] N. Auerbach, W. R. Gibbs, N. J. Ginocchio and W. B. Kaufmann, Phys. Rev. C 38 (1988) 1277

[230] N. Auerbach, W. R. Gibbs, N. J. Ginocchio and W. B. Kaufmann, Comments Nucl.Part. Phys. 20 (1991) 141 
[231] J. Gräter et al., Phys. Lett. B 471 (1999) 113

[232] J. Gräter et al., Phys. Lett. B 420 (1998) 37

[233] J. Gräter et al., Phys. Rev. C 58 (1998) 1576

[234] J. L. Clark et al., Phys. Rev. C 66 (2002) 054606

[235] A. V. Nefediev, M. G. Schepkin and H. A. Clement, Phys. Rev. C 67 (2003) 015201

[236] M. Nusseirat, M. K. Lodhi, M. O. El-Ghossain, W. R. Gibbs and W. B. Kaufmann, Phys. Rev. C 58 (1998) 2292

[237] H. C. Wu and W. R. Gibbs, Phys. Rev. C 68 (2003) 054610

[238] R. Bilger et al., Nucl. Phys. A596 (1996) 586

[239] M. G. Schepkin, O. Zabronsky and H. A. Clement, Z. Phys. A 345 (1993) 407

[240] E. Doroshkevich, W. Brodowski, J. Pätzold, H. Clement, R. Meier and G. J. Wagner, Eur. Phys. J. A18 (2003) 297

[241] L. Ya. Glozman, A. Buchmann and Amand Faessler, J. Phys. G 20 (1994) L49

[242] G. Wagner, L. Ya. Glozman, A. J. Buchmann and Amand Faessler, Nucl. Phys. A 594 (1995) 263

[243] K. Itonaga, A. J. Buchmann, G. Wagner and A. Faessler, 1 Nucl. Phys. A 609 (1996) 422

[244] I. T. Obukhovsky, K. Itonaga, G. Wagner, A. J. Buchmann and A. Faessler, Phys. Rev. C 56 (1997) 3295

[245] A. J. Buchmann. G. Wagner and Amand Faessler, Phys. Rev. C 57 (1998) 3340

[246] I. T. Obukhovsky, Amand Faessler, G. Wagner and A.J. Buchmann, Phys. Rev. C 60 (1999) 035207

[247] T. Ueda, Acta Phys. Polon. B 27 (1996) 2875

[248] T. Ueda, Nucl. Phys. A 643 (1998) 83

[249] H. Garcilazo, Phys. Rev. C 56 (1997) 1751

[250] H. Garcilazo and L. Mathelitsch, Phys. Rev. Lett. 72 (1994) 2971

[251] E. Moro, A. Valcarce, H. Garcilazo and F. Fernandez, Phys. Rev. C 54 (1996) 2085

[252] A. Valcarce, H. Garcilazo and F. Fernandez, Phys. Rev. C 54 (1996) 1010

[253] H. Garcilazo, J. Phys. G 23 (1997) 1101

[254] B. Schwesinger and N. N. Scoccola, Phys. Lett. B 363 (1995) 29

[255] E. Doroshkevich et al., Eur. Phys. J. A 18 (2003) 171 and references therein

[256] Y. Akaishi and T. Yamazaki, Phys. Rev. C 65 (2002) 044005

[257] T. Yamazaki and Y. Akaishi, Phys. Lett. B 535 (2002) 70 
[258] T. Yamazaki and Y. Akaishi, Phys. Rev. C 76 (2007) 045201

[259] T. Suzuki et al., Phys. Lett. B 597 (2004) 263

[260] M. Sato et al., Phys. Lett. B 659 (2008) 107

[261] Y. Ikeda and T. Sato, Phys. Phys. Rev. C 76 (2007) 035203

[262] N. V. Shevchenko, A. Gal and J. Mares, Phys. Rev. Lett. 98 (2007) 082301

[263] N. V. Shevchenko, A. Gal, J. Mares and J. Revai, Phys. Rev. C 76 (2007) 044004

[264] N. Barnea, A. Gal and E. Z. Liverts, Phys. Lett. B 712 (2012) 132

[265] A. Doté, T. Hyodo and W. Weise, Phys. Rev. C 79 (2009) 014003

[266] Y. Ikeda, H. Kamano and T. Sato, Prog. Theor. Phys. 124 (2010) 553

[267] A. Gal, Nucl. Phys. A 914 (2013) 270

[268] J. M. M. Hall et al., Phys. Rev. Lett.114 (2015) 132002

[269] Y. Kamiya et al., arXiv:1602.08852 [hep-ph]

[270] M. Agnello et al., Phys. Rev. Lett. 94 (2005) 212303

[271] E. Oset and H. Toki, Phys. Rev. C 74 (2006) 015207

[272] V. K. Magas, E. Oset, A. Ramos and H. Toki, Phys. Rev. C 74 (2006) 025206

[273] A. Ramos, V. K. Magas, E. Oset and H. Toki, Nucl. Phys. A 804 (2008) 219

[274] T. Yamazaki et al., Phys. Rev. Lett. 104 (2010) 132502

[275] E. Epple and L. Fabbietti, Phys. Rev. C 92 (2015) 044002

[276] A. O. Tokiyasu et al., Phys. Lett. B 728 (2014) 616

[277] Y. Ichikawa et al., Prog. Theor. Exp. Phys. 2015 (2015) 021 D01

[278] H. Garcilazo and A. Gal, Nucl. Phys. A 897 (2013) 167

[279] J. C. Berger-Chen, L. Fabietti for the HADES Collaboration, Proc. PANIC 2014, DOI: 10.3204/DESY-PROC-2014-04/101; arXiv:1410.8004[nucl-ex].

[280] Y. Sada et al., Prog. Theor.Exp. Phys. 2016 (2016) 051D01

[281] M. Iwasasaki, talk at MIN2016 workshop, Kyoto 2016, http://www2.yukawa.kyotou.ac.jp/ min2016

[282] T. Sekihara, E. Oset and A. Ramos, arXiv:1607.02058 [hep-ph]

[283] J. L. Rosner, Phys. Rev. D 33 (1986) 2043

[284] H. J. Lipkin, Phys. Lett. B 195 (1987) 484

[285] C. Gignoux, B. Silvestre-Brac and J. M. Richard, Phys. Lett. B 193 (1987) 323 
[286] A. P. Balchandran, F. Lizzi, V. G. J. Rodgers and A. Stern, Nucl. Phys. B 256 (1985) 525

[287] R. L. Jaffe and L. I. Korpa, Nucl. Phys. B 258 (1985) 468

[288] C. G. Callen and I. Klebanov, Nucl. Phys. B 262 (1985) 365

[289] U. Straub, Z. Y. Zhang, K. Bräuer, A. Faessler and S. B. Khadkikar, Phys. Lett. B 200 (1988) 241

[290] K. Nishikawa, N. Aoki and H. Hyuga, Nucl. Phys. A 534 (1991) 573

[291] M. Oka and S. Takeuchi, Nucl. Phys. A 524 (1991) 649

[292] J. Haidenbauer and U-G. Meissner, Nucl. Phys. A 881 (2012) 44

[293] J. Haidenbauer and U-G. Meissner, Phys. Lett. B 206 (2011) 100

[294] C. Wilkin, EPJ Web. Conf. 130 (2016) 01007

[295] C. Wilkin, Eur. Phys. J. A to be published; arXiv:1611.07250 [nucl-ex]

[296] D. Albers et al., Phys. Rev. Lett. 78 (1997) 1652

[297] D. Albers et al., Eur. Phys. J. A 22 (2004) 125

[298] M. Altmeier et al., Phys. Rev. Lett. 85 (2000) 1819

[299] M. Altmeier et al., Eur. Phys. J. A 23 (2005) 351

[300] F. Bauer et al., Phys. Rev. Lett. 90 (2003) 142301

[301] F. Bauer et al., Phys. Rev. C 71 (2005) 054002

[302] H. Rohdjess et al., Eur. Phys. J. A 18 (2003) 555

[303] J. Bisplinghoff, priv. comm.

[304] SAID database, http://gwdac.phys.gwu.edu/; R.A. Arndt et al., Phys. Rev. C 76,025209 (2007).

[305] S. Abd El-Samad et al., Phys. Lett. B 632 (2006) 27

[306] S. Abd El-Samad et al., Phys. Lett. B 688 (2012) 142

[307] S. Abd El-Samad et al., Eur. Phys. J. A 49 (2013) 41

[308] S. Jowzaee et al., Eur. Phys. J. A 52 (2016) 7

[309] S. Röder et al., Eur. Phys. J. A 49 (2013) 157

[310] G. Agakishiev et al., Phys. Lett. B 742 (2015) 242

[311] W. Brodowski et al., Z. Phys. A 355 (1996) 5

[312] W. Brodowski et al., Phys. Lett. B 550 (2002) 147

[313] H. Clement et al., Prog. Part. Nucl. Phys. 36 (1996) 369

[314] S. Abd El-Bary et al., Eur. Phys. J. A 37 (2008) 267 
[315] A. Erhardt, Dissertation Univ. Tübingen 2009

[316] A. Faessler, A. J. Buchmann and M. I. Krivoruchenko, Phys. Rev. C 57 (1998) 1458

[317] L. G. Dakhno et al., Sov. J. Nucl. Phys. 37 (1983) 540

[318] C. D. Brunt, M. J. Clayton and B. A. Wetswood, Phys. Rev. 187 (1969) 1856

[319] F. Shimizu et al., Nucl. Phys. A 386 (1982) 571

[320] V. V. Sarantsev et al., Phys. At. Nucl. 70 (2007) 1885

[321] A. M. Eisner et al., Phys. Rev. 138 (1965) B670

[322] E. Pickup, D. K. Robinson and E. O. Salant, Phys. Rev. 125 (1961) 2091

[323] W. Brodowski et al., Phys. Rev. Lett. 88 (2002) 192301

[324] J. Johanson et al., Nucl. Phys. A 712 (2002) 75

[325] J. Pätzold et al., Phys. Rev. C 67 (2003) 052202

[326] T. Skorodko et al., Eur. Phys. J. A 35 (2008) 317

[327] T. Skorodko et al., Phys. Lett. B 679 (2009) 30

[328] F. Kren et al., Phys. Lett. B 684 (2010) 110 and Phys. Lett. B 702 (2011) 312; arXiv:0910.0995[nuclex]

[329] T. Skorodko et al., Phys. Lett. B 695(2011) 115

[330] T. Skorodko et al., Eur. Phys. J. A 47 (2011) 108

[331] P. Adlarson et al., Phys. Lett. B 706 (2011) 256

[332] S. Abd El-Samad et al., Eur. Phys. J. A 42 (2009) 159

[333] J. Bystricky et al., J. Physique 48 (1987) 1901

[334] L. Alvarez-Ruso, E. Oset, E. Hernandez, Nucl. Phys. A 633 (1998) 519

[335] X. Cao, B.-S. Zou and H.-S. Xu, Phys. Rev. C 81 (2010) 065201

[336] M. Bashkanov and H. Clement, Eur. Phys. J. A 50 (2014) 107

[337] A. Abashian, N. E. Booth and K. M. Crowe, Phys. Rev. Lett. 5 (1960) 258; 7 (1961) 35; Phys. Rev. 132 (1963) 2296

[338] I. Bar-Nir et al., Nucl. Phys. B 54 (1973) 17

[339] A. Abdivaliev et al., Sov. J. Nucl. Phys. 29 (1979) 796

[340] R. J. Homer et al., Phys. Rev. Lett. 9 (1964) 72

[341] J. H.Hall et al., Nucl. Phys. B 12 (1969) 573

[342] J. Banaigs et al., Phys. Lett. B43 (1973) 535 
[343] J. Banaigs et al., Nucl. Phys. B 67 (1973) 1

[344] J. Banaigs et al., Nucl. Phys. B 105 (1976) 52

[345] F. Plouin et al., Nucl. Phys. A 302 (1978) 413

[346] F. Plouin, P. Fleury, C. Wilkin, Phys. Rev. Lett. 65 (1990) 690

[347] R. Wurzinger et al., Phys. Lett. B 445 (1999) 423

[348] for a review see A. Codino and F. Plouin, LNS/Ph/94-06

[349] T. Risser and M. D. Shuster, Phys. Lett. B 43 (1973) 68

[350] M. Bashkanov et al., Phys. Lett. B 637 (2006) 223

[351] M. Abdel-Bary et al., Eur. Phys. J. A 29 (2006) 353

[352] R. Bilger et al., Nucl. Instr. Meth. A 457 (2001) 64

[353] S. Barshay and G. M. Temmer, Phys. Rev. Lett. 12 (1964) 728

[354] H. Clement, M. Bashkanov and T. Skorodko, EPJ Web of Conf. 97 (2015) 00010

[355] P. Adlarson et al., Eur. Phys. J. A 52 (2016) 147

[356] T. Tsuboyama, F. Sai, N. Katayama, T. Kishida and S. S. Yamamoto, Phys. Rev. C 62 (2000) 0340011

[357] D. V. Bugg, Eur. Phys. J. A 50 (2014) 104

[358] I. Bar-Nir et al., Nucl. Phys. B 54 (1973) 17

[359] A. Abdivaliev et al., Sov. J. Nucl. Phys. 29 (1979) 796; Nucl. Phys. B 168 (1980) 385

[360] H. Clement, M. Bashkanov and T. Skorodko, Phys. Scr. T 166 (2015) 014016

[361] M. Platonova and V. Kukulin, Phys. Rev. C 87 (2013) 025202

[362] M. Bashkanov, H. Clement and T. Skorodko, Nucl. Phys. A 958 (2017) 129

[363] L. Alvarez-Ruso, PhD thesis Univ. Valencia 1999

[364] G. Fäldt and C. Wilkin, Phys. Lett. B 701 (2011) 619

[365] M. Albaladejo and E. Oset, Phys. Rev. C 88 (2013) 014006

[366] G. Agakishiev et al., Phys. Lett. B 750 (2015) 184

[367] M. Bashkanov, H. Clement and T. Skorodko, Eur. Phys. J. A 51 (2015) 87

[368] R. L. Workman, W. J. Briscoe and I. I. Strakovsky, Phys. Rev. C 93 (2016) 045201

[369] R. Workman, EPJ Web Conf. 81 (2014) 02023

[370] R. L. Workman, W. J. Briscoe and I. I. Strakovsky, Phys. Rev. C in press; arXiv:1609.01741 [nucl-th] 
[371] J. Ball et al., Nucl. Phys. A 559 (1993) 489

[372] A. de Lesquen et al., Eur. Phys. J. C 11 (1999) 69

[373] Y. Makdisi et al., Phys. Rev. Lett. 45 (1980) 1529

[374] C. R. Newsom et al., Phys. Rev. C 39 (1989) 965

[375] J. Arnold et al., Eur. Phys. J. C 17 (2000) 67

[376] J. Ball et al., Nucl. Phys. B 286 (1987) 635

[377] M. W. McNaughtton et al., Phys. Rev. C 48 (1993) 256; 53 (1996) 1092

[378] G. Glass et al., Phys. Rev. C 47 (1993) 1369

[379] K. Wijesooriya et al., Phys. Rev. Lett. 86 (2001) 2975

[380] A. Faessler, A. J. Buchmann and M. I. Krivoruchenko, Phys. Rev. C 57 (1998) 1458

[381] M. I. Krivoruchenko et al., Phys. At. Nucl. 74 (2011) 371 and references therein

[382] A. W. Thomas et al., EPJ Web Conf 63 (2013) 03004

[383] S. Kagiyama, A. Nakamura and T. Omodaka, Z. Phys. C 56 (1992) 557

[384] A. Akmal, V. R. Pandharipande and D. G. Ravenhall, Phys. Rev. C 58 (1998) 1804

[385] P. Adlarson et al., Phys. Rev. C 91 (2015) 015201

[386] P. Adlarson et al., Phys. Rev. C 86 (2012) 032201(R)

[387] R. J. Porter et al., Phys. Rev. Lett. 79 (1997) 1229

[388] G. Agakichiev et al., Phys. Lett. B 690 (2010) 118

[389] J. L. Ping, H. X. Huang, H. R. Pang, F. Wang and C. W. Wong, Phys. Rev. C 79 (2009) 024001 and references therein

[390] Hua-Xing Chen, Er-Liang Cui, Wei Chen, T. G. Steele and Shi-Lin Zhu, Phys. Rev. C 91 (2015) 025204

[391] W. Park, A. Park and S. H. Lee, Phys. Rev. D 92 (2015) 014037

[392] K. Sasaki for the HALQCD collaboration, talk at the MPMBI workshop, Sendai 2016, http://www.lns.tohoku.ac.jp/workshop/c013/slides/MPMBI-sasaki.pdf

[393] H. Garcilazo, F. Fernandez, A. Valcarce and R. D. Mota, Phys. Rev. C 56 (1997) 84

[394] A. Valcarce, H. Garcilazo, R. D. Mota and F. Fernandez, J. Phys. G 27 (2001) L1

[395] Y. Dong, P. Shen, F. Huang and Z. Zhang, Phys. Rev. C 91 (2015) 064002

[396] F. Huang, P. N. Shen, Y. B. Dong and Z. Y. Zhang, Sci. China-Phys. Mech. Astron. 59 (2016) 622002

[397] Y. Dong, F. Huang, P. Shen and Z. Zhang, Phys. Rev. C 94 (2016) 014003 
[398] J. A. Niskanen, arXiv:1610.06013 [nucl-th]

[399] C. S. An and H. Chen, Eur. Phys. J. A 52 (2016) 2

[400] F. Huang, P. N. Shen, Y. B. Dong and Z. Y. Zhang, JPS Conf. Proc. 10 (2016) 022002

[401] A. Gal priv. comm. and to be published.

[402] P. Adlarson et al., to be published.

[403] F. Piccinini, talk at the International School of Nuclear Physics, 37th course, Erice (2015)

[404] C. Bignamini, B. Grinstein, F. Piccinini, A. D. Polosa and C. Sabelli, Phys. Rev. Lett. 103 ( 2009 ) 162001

[405] Chun Wa Wong, Phys. Rev. C 61 (2001) 064011

[406] Di Qing, He-Ming Sun and Fan Wang, Chin. Phys. Lett. 18 (2001) 885

[407] M. Bashkanov, priv. comm.

[408] A. Fix and H. Arenhövel, Eur. Phys. J. A 25 (2005) 115

[409] M. Egorov and A. Fix, Nucl. Phys. A 933 (2015) 104

[410] M. Guenther, master thesis, Univ. Basel 2015

[411] T. Ishikawa et al., arXiv:1610.05532 [nucl-ex]

[412] S. Teis, W. Cassing, M. Effenberger, A. Hombach, U. Mosel and Gy. Wolf, Z. Phys. A 356 (1997) 421 and references therein

[413] K. U. Abraamyan et al., Phys. Rev. C 80 (2009) 034001

[414] K. U. Abraamyan et al., Eur. Phys. J. A 52 (2016) 259

[415] M. Bashkanov et al., Int. J. Mod. Phys. A (2005) 554

[416] Q. B. Li and P. N. Shen, J. Phys. G 26 (2000) 1207

[417] L. M. Andronenko et al., AIP Conf. Proc. 221 (1990) 216

[418] P. Adlarson et al., Phys. Lett. B 762 (2016) 455

[419] V. Komarov et al., Phys. Rev. C 93 (2016) 065206

[420] R. Bilger et al., Nucl. Phys. A 693 (2001) 633

[421] R. Hanbury Brown, R. Q. Twiss, Nature 10 (1956) 1046

[422] K. Morita, A. Ohnishi, F. Etminan and T. Hatsuda, Phys. Rev. C 94 (2016) 031901

[423] Q. B. Li and P. N. Shen, Eur. Phys. J. A 8 (2000) 417

[424] F. Etminan et al., Nucl. Phys. A 928 (2014) 89

[425] M. Karliner, H. J. Lipkin and N. A. Tornquist, arXiv:1109.3472 
[426] M. Karliner, H. J. Lipkin and N. A. Tornquist, Nucl. Phys. B Proc. Suppl. 225 - 227 (2012) 102

[427] M. Karliner and S. Nussinov, J. High Energy Phys. 07 (2013) 153

[428] M. Karliner and J. L. Rosner, Phys. Rev. Lett. 115 (2015) 122001

[429] A. Gal, H. Garcilazo, A. Valcarce and T. Fernandez-Carames, Phys. Rev. D 90 (2014) 014019

[430] A. Valcarce, H. Garcilazo, F. Fernandez and P. Gonzales, Rep. Prog. Phys. 68 (2005) 965

[431] M. Bayar, C. W. Xiao, T. Hyodo, A. Dote, M. Oka and E. Oset, Phys. Rev. C 86 (2012) 044004 